\title{
Theoretical description of Ti and Ti alloys from first principles
}

Natalia Skripnyak 
Linköping studies in science and technology

Dissertation No. 2064

\title{
Theoretical description of Ti and Ti alloys from first principles
}

\author{
Natalia Skripnyak
}

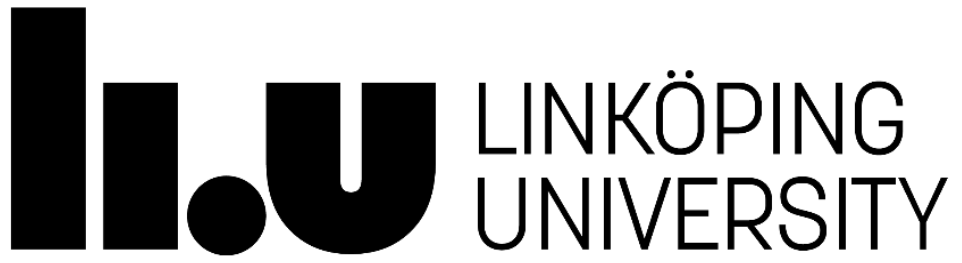

Theory and Modelling

Departament of Physics, Chemistru and Biology (IFM)

Linköping University, SE-581 83 Linköping, Sweden

Linköping 2020 
(C) Natalia Skripnyak, 2020

Printed in Sweden by LiU-Tryck 2020

ISSN 0345-7524

ISBN 978-91-7929-871-5 
Theoretical description

of $\mathrm{Ti}$ and $\mathrm{Ti}$ alloys from first principles

By

Natalia Skripnyak

May 2020

ISBN 978-91-7929-871-5

Linköping studies in science and technology Dissertation

No. 2064

ISSN 0345-7524

\begin{abstract}
Modern world is known for many advanced technologies and solutions to complex problems. Technical progress runs at high speed. In order to most effectively use materials, given to us by Nature, it is important to know their properties. To do laboratory experiments is often too expensive and time consuming. Therefore, it is very important to possess the knowledge and capabilities of studying materials properties without actual experiments. I use different methods based on the laws of Quantum mechanics to conduct my investigations. In this work I studied from first principles properties of titanium and titanium alloys that are of potential interest for various applications. Titanium was chosen because of its unique properties, which are both useful and reveal interesting physics. First, I investigated elastic properties using density functional theory (DFT) in different implementations, such as the projector augmented wave (PAW) and the exact muffin-tin orbitals (EMTO) methods. The single crystal's elastic constants $\mathrm{C}_{\alpha \beta}$ of pure Ti, Ti-V, and Ti-Ni-Al alloys were obtained by calculating the total energy as a function of appropriate strains or stress-strain relations. Disordered substitutional alloys were modeled using a special quasi-random structure (SQS) technique combined with PAW as well as the coherent potential approximation (CPA) combined with EMTO. The concentration dependence of $\mathrm{C}_{\alpha \beta}$ and also the family of material characteristics, such as Young's modulus E, bulk modulus B, shear modulus G, Cauchy pressure $\mathrm{P}_{\mathrm{c}}$, Pugh's coefficient $\mathrm{k}$, and Poisson's coefficient $v$ for the TiV system were estimated and discussed. The elastic properties of alloys in the Ni-AlTi system were also calculated and analyzed, as well as the temperaturedependent elastic constants of pure Ti. The influence of the amount of $\mathrm{V}$ on
\end{abstract}


the mechanical phases stability of body-centered cubic (bcc) Ti-V alloys was studied. It was found that Ti-rich Ti-V alloys are mechanically unstable in the bcc phase, but at higher concentration of $\mathrm{V}$ in the system the mechanical stability is increased. It was found that the Ni-Al-Ti system is mechanically stable in accordance with the requirements of mechanical stability for a cubic crystal. The first-principles calculations yielded solution enthalpies for B2 and bec solid solution alloys. The enthalpies of bcc Ti-V alloys were calculated from first principles at 0 and $1300 \mathrm{~K}$ as a function of concentration using static and molecular dynamics simulations. The enthalpy curves for the B2 Ti-V alloys were described as a function of the $\mathrm{V}$ concentration by using the calculated solution enthalpies. The enthalpies of the $\beta$-phase Ti-V alloys decrease with increasing concentration of vanadium in the range from 0 to 1 . Next, selfdiffusion in pure Ti was studied at high temperature using classical and ab initio molecular dynamics. We reveled a physical mechanism entailing a rapid collective movement of numerous (from two to dozens) neighboring titanium atoms along tangled closed-loop paths in defectfree crystal regions. Further, we addressed the effect of atomic relaxations on the formation enthalpy and the size of the tetra and octa voids in the body-centered cubic (bcc) high entropy alloys (HEA), where one of the principal elements is Ti. These are the alloys with 5 different components in equal proportions, which recently become the objects of extensive research due to their interesting properties, such as, for example, combined toughness and plasticity as well as corrosion resistance. We found that the relaxations are crucial and can change the energetically preferable distribution of elements in the periodic bcc lattice from segregated to random-alloy-like. The tetra and octa voids in HEAs can accommodate interstitial impurities that can be of interest to improve the alloy properties. We found that the distribution of void volumes due to atomic relaxations can be described by a set of Gaussians, whose number depends on the type of the void and the atomic distribution (random vs segregated). It could also be important that the largest volumes of the voids due to atomic relaxations are increased by nearly $25 \%$.

Keywords: (Ti; Ti alloys; DFT; molecular dynamics; thermodynamic properties; elastic properties)

\author{
Departament of Physics, Chemistru and Biology (IFM) \\ Linköping University \\ SE-581 83 Linköping, Sweden
}




\section{Populärvetenskaplig sammanfattning}

Dagens samhälle präglas av avancerad teknik och lösningar på komplexa problem. Den teknologiska utvecklingen sker fort. För att effektivast kunna använda naturens alla material, är det viktigt att förstå deras egenskaper. Att utföra experiment är ofta dyrt och tidskrävande. Därför är det väsentligt att besitta kunskapen och förmågan att kunna studera materialegenskaper utan faktiska experiment. Jag använder olika metoder baserade på kvantmekanikens lagar för att utföra mina undersökningar. I denna avhandling studerar jag från första-princip-beräkningar egenskaper hos titan och titanlegeringar som är av potentiellt intresse för olika tillämpningar. Titan valdes på grund av dess unika egenskaper som både är användbara och som även uppenbarar intressant fysik. Till en början undersökte jag elastiska egenskaper inom ramen för täthetsfunktionalteori (DFT) genom att applicera olika implementeringar, såsom "projector augmented wave (PAW)" och "exact muffin-tin orbitals (EMTO)" metoderna. De enkelkristallina elasticitetskonstanterna C $\alpha \beta$ för rent Ti, Ti-V, och Ti-Ni-Al legeringar erhölls genom att beräkna totala energin som funktion av lämpliga töjningar eller "spänningtöjning/dragprovs"relationer. Oordnade substitutionella legeringar modellerades av speciella kvasislumpmässiga strukturer (SQS) kombinerat med PAW, och även med den koherenta potentialapproximationen (CPA) kombinerat med EMTO. Koncentrationsberoendet av $\mathrm{C} \alpha \beta$ och andra materialegenskaper, såsom Youngs modul E, bulkmodul B, skjuvmodul G, Cauchy-tryck Pc, Pugh's koefficient $\mathrm{k}$, och Poissons konstant $v$, uppskattades och diskuterades för systemet TiV. De elastiska egenskaperna för Ni-Al-Ti legeringar beräknades och analyserades, såväl som de temperaturberoende elastiska konstanterna för rent Ti. Influensen som mängden $\mathrm{V}$ har på mekanisk fasstabilitet hos rymdcentrerad kubiska (bcc) Ti-V legeringar undersöktes. Det visade sig att Ti-rika Ti-V legeringar är mekaniskt instabila i bcc-fasen, men vid högre koncentration av V i systemet ökar den mekaniska stabiliteten. Ni-Al-Ti systemet konstaterades vara mekaniskt stabilt, i linje med kraven för mekanisk stabilitet för en kubisk kristall. Entalpierna för bcc Ti-V legeringar beräknades från förstaprinciper vid 0 och $1300 \mathrm{~K}$ som funktion av koncentration genom att använda statiska och molekyldynamiksimuleringar. Entalpikurvorna för Ti-V legeringarna beskrevs som funktion av V-koncentrationen genom att använda de beräknade blandningsentalpierna. Entalpierna för $\beta$-fas Ti- 
$\mathrm{V}$ legeringar minskar med ökande koncentration av vanadin $\mathrm{i}$ intervallet 0 till 1. Följaktligen, själv-diffusion $\mathrm{i}$ rent $\mathrm{Ti}$ studerades vid hög temperatur med klassisk och ab initio molekyldynamik. Vi fann en fysisk mekanism innehållande en snabb kollektiv rörelse av flera (från två till dussintal) närliggande titanatomer längs knutna slingor $\mathrm{i}$ defektfria kristallregioner. Dessutom riktade vi in oss på effekten av atomrelaxationer på formationsentalpi och storleken på tetraediska och oktaedriska hål i bcc högentropilegeringar (HEA), där ett av huvudämnena är Ti. Denna typ av legeringar består av 5 olika komponenter, med lika andelar, som nyligen har alstrat ett stort forskningsintresse på grund av deras intressanta egenskaper, såsom exempelvis kombinationen hårdhet och plasticitet tillika korrosionsbeständighet. Vi fann att relaxationer är kritiska och kan påverka den energimässigt fördelaktiga distributionen av ämnen i det periodiska bcc gittret från segregerat till oordnat tillstånd. Tetraediska och oktaedriska hål i HEAs kan rymma interstitiella orenheter som kan vara av intresse vid förbättring av legeringsegenskaper. Vi kom fram till att fördelningen av hålvolymer, kommande från atomrelaxationer, kan beskrivas av en mängd Gaussiska sådana, vars nummer beror på typen av hål samt den atomistiska distributionen (oordnad vs segregerad). Det kan också vara viktigt att de största hålvolymerna, kommande från atomrelaxationer, ökar med nästan $25 \%$. 


\section{Acknowledgement}

This thesis is a result of my Ph.D. work at the Theoretical Physics Division at IFM, Linkoping University. First of all, I would like to thank my main supervisor, Prof. Igor Abrikosov, for the opportunity to study and work in a pleasant group, for helping and indicating the right direction in research.

I would also like to thank my co-supervisor Irina Yakimenko for her help, teaching and sincere conversations.

I am very grateful to my co-supervisor Sergey Simak for the training and support.

I want to thank my colleagues for their help in work and my numerous friends for the good time that we spent together. 


\section{Contents}

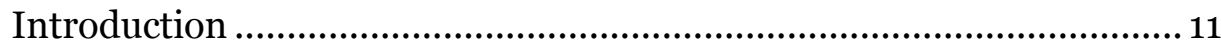

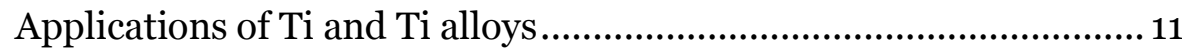

Thermodynamic, elastic properties, and diffusion ...........................12

Theoretical Background ............................................................................15

Density functional theory (DFT) ......................................................15

Many-particle problem ............................................................. 15

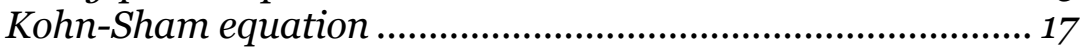

Exchange-correlation functional.............................................. 19

The Exact Muffin-Tin Orbitals method (EMTO) ................................19

Projector Augmented Wave method (PAW) ........................................ 20

Molecular dynamic (MD) ................................................................21

Special Quasirandom Structures (SQS) ........................................... 23

Elastic Properties: general theory …........................................................ 25

Single-crystal elastic constants ............................................... 25

Elastic moduli of polycrystalline alloys ................................... 28

Calculations of elastic properties of $\mathrm{Ti}$ and its alloys ......................... 34

Details of EMTO-CPA calculations........................................... 34

Details of PAW-SQS calculations ............................................. 34

Details of $M D$ calculations ....................................................... 35

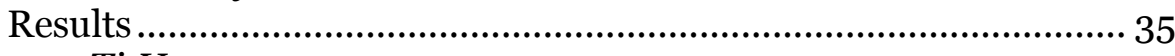

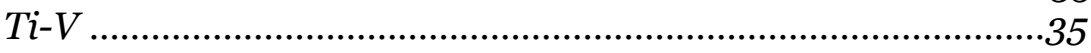

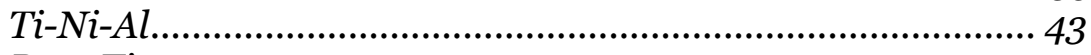

Pure Ti ............................................................................. 45

Elastic anisotropy ........................................................................... 46

Mechanical stability …......................................................................... 49

Details of calculations ....................................................................... 49

Results ....................................................................................... 49

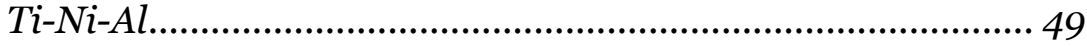

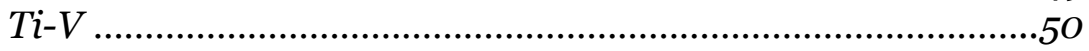

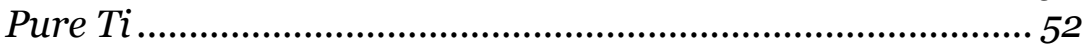

Ti-V at high temperature ..................................................... 55

Mixing Enthalpy ............................................................................... 57

Details of calculations .................................................................... 57

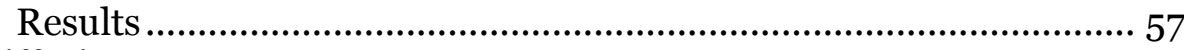

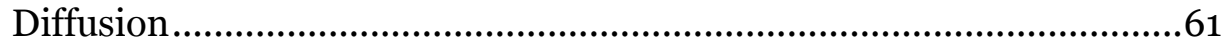

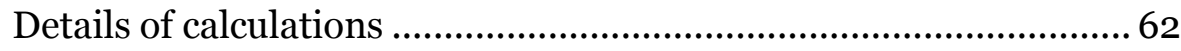

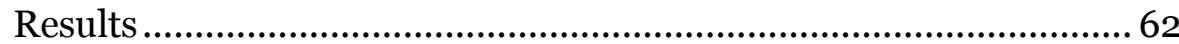

Non-Arrhenius behavior of diffusion coefficient in bcc Ti...... 62 
Self-diffusion in bec $\mathrm{Ti}$............................................................63

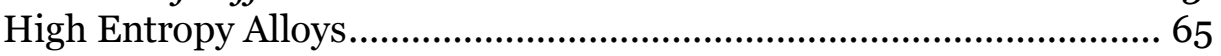

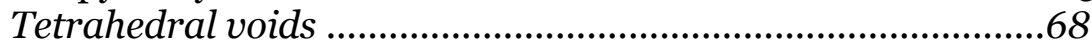

Octahedral voids ................................................................70

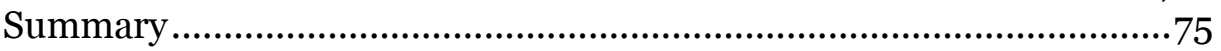

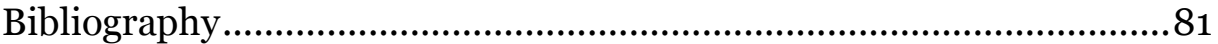



Chapter 1

\section{Introduction}

\section{Applications of $\mathrm{Ti}$ and $\mathrm{Ti}$ alloys}

$\mathrm{Ti}$ and Ti-alloys are well known as light and high-strength materials and are of practical interest for the aerospace and automotive industry. $\mathrm{Ti}$ is the most important structural material in aerospace related production and shipbuilding.

At conditions that happen in applications it usually exists in either of three crystalline modifications: $\alpha$-Ti with the hexagonal close-packed (hcp) lattice, $\beta$-Ti with the body-centered cubic (bcc) packing, and $\omega$-Ti with a hexagonal primitive lattice. The $\beta$-phase is always more durable with high resilience and is also easily processed. $\beta$-Ti attracted particular attention because of its workability and higher strength, variable elastic moduli, excellent corrosion and wear resistance, light weight and good ductility.

Vanadium is one of the so-called $\beta$-stabilizers of titanium alloys. That is, the alloying with vanadium makes it possible to stabilize the hightemperature bcc phase of titanium at temperatures below $1155 \mathrm{~K}$, which is often beneficial for the mechanical properties of titanium alloys in structural elements operating in a wide range of temperatures. Another advantage of vanadium as an alloying element in titanium alloys is the absence of eutectoid reactions and intermetallic phases in the Ti-V system. This almost eliminates the appearance of brittleness in cases of errors in carrying out technological processes associated with heating. In addition, Ti-V alloys have a very narrow range of crystallization [1]-[3]. Moreover, it is known that vanadium-rich alloys with $\mathrm{Ti}$ additions combine low temperature strength and high ductility with high strength at elevated temperature and low creep [4].

As mentioned above, the Ti-V alloys are of practical interest for hightemperature structural elements of the aerospace and automotive industries. Also they are important for the development of nuclear reactors. In particular, these alloys are suitable to $4^{\text {th }}$ generation nuclear reactors. Nuclear reactors of the $4^{\text {th }}$ generation on fast neutrons are considered as systems including both the reactor and subsystems of processing(recycling) nuclear fuel. Such new system should have higher operating performance than previous generations of nuclear reactors. They must ensure competitiveness, safety and reliability and protection against the proliferation of nuclear materials. Interest in the properties of $\beta$-titanium alloys has further increased in view of their application to 
create elements of designs by using Selective Laser Sintering (SLS) technology [5]. Applicability of Ti-V alloys for hydrogen storage has been considered as well [6]-[8].

Ti alloys are materials with a wide range of elastic constants. Particular ranges of the elastic moduli are required by particular applications. For example, there are many applications, which require materials with low Young's modulus. For biomedical application the materials with relativity low values of Young's modulus close to the value of human tissue are needed in order to ensure biomedical compatibility. $\beta$-Ti alloys in the body-centered cubic (bcc) structure show lower Young's modulus than that of $\alpha$ in the hexagonal close-packed (hcp) structure and $\alpha+\beta$ titanium alloys [9]. Therefore Ti-V alloys in the bcc structure are good candidates. Though vanadium is not used for fabrication of biomedical implants because of its toxicity, its Ti-based alloys with low elastic moduli are needed as materials for stents and other biomechanical devices, where the materials with high elastic moduli would harm human tissues. Usually such devices have coatings with special functions that in particular protect humans from toxic content. Besides, the materials with low elastic moduli could be useful for sensor systems, such as motion detectors and shock absorbers that provide damping of vibrations. Systems of the spring type for damping of vibrations are very important for the work of many devices that are subject to vibrations, for example for aircraft engines. The higher the speed of the aircraft the higher is the speed of the shaft movement in a turbine system and the frequencies of vibrations that are transferred to all the parts of the machinery. The higher the elastic modulus the smaller the displacement. The knots at large displacements cause high stresses. To decrease stresses, one needs to increase the sizes of the devices, and that is impractical. With low elastic moduli, the elements of the spring type can be decreased in size to provide bigger displacement of the system and larger damping of energy.

\section{Thermodynamic, elastic properties, and diffusion}

In recent years, first-principles theoretical studies have become an important tool in the field of materials science to understand and predict different properties and behavior of materials. Thermodynamic stability of crystal phases can nowadays be estimated at given experimental conditions by calculating enthalpy or Gibbs free energy. This is done both at $\mathrm{o} \mathrm{K}$ and higher temperatures.

Elastic constants belong to important characteristics of solids, being a measure of the strength of chemical bonding. Moreover, the results of the calculation of Young's modulus illuminate the possibility of the 
highly non-linear concentration behavior of the Young's modulus, which is difficult to predict based on either linear interpolations between values for the pure elements or by extrapolation of values obtained in the mechanically stable regions and are of direct relevance for materials design. This makes it possible to search for the optimal content of the alloying component, which provides both mechanical stability and a given value of the Young's modulus. For engineering applications, it is often sufficient to use just Young's modulus, however, if one wants to address the strength characteristics, shear modulus is also very important, as follows from the general theory of physical mechanisms in materials subject to deformations and stresses.

Shear modulus (G) is one of the key parameters for analyses of physical mechanisms of the development of defect structures. All real parameters connected to plastic deformation and cutting are normalized to shear modulus. The need in corresponding theoretical values is high. However theoretical knowledge of shear moduli is practically absent. Modeling of the change of moduli is an important task. Poisson coefficient $(v)$ is less sensitive to the changes of the lattice constants. Shear modulus enters practically any theoretical model for predicting defects. When dislocation mechanisms are acting, the predicted $\mathrm{G}$ determines the shear velocity of sound that limits the speed of the dislocation movement. If there is a theoretical prediction how the structure affects the modulus, when it changes, the shear velocity also changes leading to the change also in the longitudinal velocity and Young's modulus.

Questions concerning control over crystalline phases is currently a hot topic of research. Diffusion processes determine the kinetics of all metallurgical processes, they describe the decomposition of solid solutions, the release of particles in alloys, oxidation, creep, etc. Understanding and making proper use of these processes requires clear and accurate knowledge of diffusion. Also, the study of diffusion is important for understanding the mechanisms occurring in the crystal. The characteristics of point defects are related to the diffusion coefficient. 

Chapter 2

\section{Theoretical Background}

$A b$ initio (first-principles) calculations make it possible to predict the behavior of a material based on quantum-mechanical considerations without requiring experimental knowledge of the fundamental properties of the material.

Electronic structure calculations have become a very useful tool in the field of computational materials science. Many physical or chemical properties of materials can be predicted directly from the solutions of the fundamental quantum-mechanical equations for the electrons.

\section{Density functional theory (DFT)}

Density functional theory (DFT) is a method for calculating the electronic structure of many-particle systems.

The main idea of the DFT is to replace a multi-electron problem with an effective one-electron problem. Therefore, the incredible task to calculate multi-electron wave function is replaced by a much simpler task to calculate the electron density. Such a replacement allows for efficient procedures that can be coded and run on existing (super)computers. All properties of the system in the ground state are considered as density functionals.

\section{Many-particle problem}

The electronic and nuclei system in a material can be described within the non-relativistic quantum mechanics by the many-particle Schrödinger equation. Solutions to the Schrödinger equation describe not only molecular, atomic, and subatomic systems, but in principle the whole macroscopic systems.

The wave function defines the state of the system at each spatial position, and time. According to the time-independent Schrödinger equation, the many-particle wave function $\Psi=\left(\mathrm{r}_{1}, \mathrm{r}_{2}, \cdots \mathrm{r}_{\mathrm{N}}, \mathrm{R}_{1}, \mathrm{R}_{2}, \cdots, \mathrm{R}_{\mathrm{M}}\right)$ which is a function of all the positions ( $r_{N}$ for electrons, $R_{M}$ for ions) in the system is given via the linear operator equation:

$$
\widehat{H} \Psi=\mathrm{E} \Psi \text {, }
$$

where $\mathrm{E}$ is the energy.

Above I skip the spin-dependence of $\Psi$, which in principle can be added on top of the Schrödinger equation. As all the materials in this study are non-magnetic, that is justified.

The Hamiltonian operator $\widehat{H}$ of such a system is expressed as: 


$$
\widehat{H}=\mathrm{T}_{\mathrm{e}}+\mathrm{T}_{\mathrm{n}}+\mathrm{V}_{\mathrm{ee}}+\mathrm{V}_{\mathrm{en}}+\mathrm{V}_{\mathrm{nn}}
$$

The first two terms represent the kinetic energy of nuclei and electrons, respectively. $V_{\text {en }}$ is the Coulomb interaction between the electrons and the nuclei, while the other two terms account for the electron-electron and the nucleus-nucleus interactions, respectively. The corresponding expressions are:

$$
\begin{array}{ll}
\mathrm{T}_{\mathrm{e}}=\frac{1}{2 m} \sum_{i=1}^{N e} p_{i}^{2} & \text { for kinetic energy of electrons } \\
\mathrm{T}_{\mathrm{n}}=\frac{1}{2} \sum_{k=1}^{N n} \frac{1}{M_{k}} P_{k}^{2} & \text { for kinetic energy of the nuclei } \\
\mathrm{V}_{\mathrm{ee}}=\frac{e^{2}}{2} \sum_{i, j}^{\prime} \frac{1}{\left|r_{i}-r_{j}\right|} & \text { for electron-electron interactions } \\
\mathrm{V}_{\mathrm{nn}}=\frac{e^{2}}{2} \sum_{k \neq l} \frac{Z_{k} Z_{l}}{\left|R_{k}-R_{l}\right|} & \text { for nucleus-nucleus interactions } \\
\mathrm{V}_{\mathrm{en}}=-e^{2} \sum_{i, k} \frac{Z_{k}}{\left|r_{i}-R_{k}\right|} & \text { for electron-nucleus interactions }
\end{array}
$$

$\mathrm{m}$ and e are the electron mass and charge. $\mathrm{p}_{\mathrm{i}}$ and $\mathrm{P}_{\mathrm{k}}$ are the moment operators of electron $i$ and nucleus $k$, respectively. The summations are done over all $\mathrm{N}_{\mathrm{e}}$ electrons and $\mathrm{N}_{\mathrm{n}}$ nuclei. Therefore, if a system of stationary ions is considered and $\mathrm{T}_{\mathrm{n}}=0$ can be assumed,

$$
\widehat{H}=\sum_{i} \frac{-\hbar^{2}}{2 m} \nabla_{i}^{2}-\frac{1}{2} \sum_{i \neq j} \frac{e^{2}}{\left|r_{i}-r_{j}\right|}+\frac{e^{2}}{2} \sum_{k \neq l} \frac{Z_{k} Z_{l}}{\left|R_{k}-R_{l}\right|}-e^{2} \sum_{i, k} \frac{Z_{k}}{\left|r_{i}-R_{k}\right|},
$$

describing electrons with mass $m_{e}$ and ions with mass $M_{k}$. Planck constant, and $\mathrm{Z}_{\mathrm{k}}$ is the nuclear charge of ion $\mathrm{k}$.

To simplify the problem, Born and Oppenheimer proposed to consider nuclei that make up the system under consideration as immobile particles for electrons, the speed of which allows us to assume that they instantly reach the distribution of the ground state for any positions of the nuclei. [10]. The mass of the nucleus significantly exceeds the mass of the electron, as a result of which the speed of the nuclei is small relative to the speed of the electrons. Therefore, in the Born-Oppenheimer approximation, nuclei are considered fixed and only electron motion is taken into account. The potential energy of interaction of electrons with ions is considered as electrons in an external field. Potential energy of nuclei is defined as a constant external potential.

The density functional theory is based on two theorems that were formulated by Hohenberg and Kohn [11]: 


\section{Theorem 1}

There is a one-to-one correspondence between the density of the ground state of the electronic subsystem $\mathrm{n}_{\mathrm{o}}(\mathrm{r})$ located in the external potential of atomic nuclei and the potential of the nuclei themselves $\mathrm{V}_{\text {ext }}(\mathrm{r})$.

\section{Theorem 2}

The energy of the electronic subsystem E(n), recorded as the functional of electron density $n(r)$, has a minimum equal to the energy of the ground state.

Thus, the problem with $\mathrm{N}_{\mathrm{n}}$ electrons is replaced by a system of $\mathrm{N}_{\mathrm{n}}$ oneelectron Schrödinger equations.

\section{Kohn-Sham equation}

A solid is considered as a system consisting of a large number of indistinguishable electrons interacting with each other, held together by a lattice of atomic nuclei. The main idea of the method is to use the concept of electron density in the ground state, its distribution is described by the Kohn-Sham equation, which is one-particle Schrödinger equation.

$$
\left[\frac{-\hbar^{2}}{2 m} \nabla^{2}+V_{e x t}(r)+V_{H}(r)+V_{X C}(r)\right] \psi_{i}(r)=\varepsilon_{i} \psi_{i}(r)
$$

where $\mathrm{V}_{\mathrm{H}}=\int \frac{e^{2} n\left(\vec{r}^{\prime}\right)}{\left|\vec{r}-\vec{r}^{\prime}\right|} d^{3} r^{\prime}$ is the Hartree term, which describes the electrostatic electron-electron Coulomb interaction, $\mathrm{V}_{\mathrm{xc}}[\mathrm{n}(\mathrm{r})]=\frac{\delta E_{x c}[n(r)]}{\delta n(r)}$ is the exchange-correlation potential.

Self-consistent system of Kohn-Sham equations can be solved using an iterative procedure of successive approximations. First, starting from the initial approximation for $\mathrm{n}(\mathrm{r})$, the corresponding term $\mathrm{U}$

$$
\mathrm{U}(\mathrm{r})=\mathrm{V}_{\mathrm{ext}}+\mathrm{V}_{\mathrm{H}}+\mathrm{V}_{\mathrm{xc}}[\mathrm{n}(\mathrm{r})]
$$

is calculated, for which the Kohn - Sham equations (Eq. (2.4)) are then solved, from which $\psi_{i}$ are obtained. From here one can obtain a new approximation for density $\mathrm{n}(\mathrm{r})$

$$
\mathrm{n}(\mathbf{r})=\sum_{i}^{o c c}\left|\psi_{i}(\boldsymbol{r})\right|^{2}
$$


to use it again in Eq. 2.4 and continue this procedure until the input and output densities are the same. [12].

The practical way of solving Kohn-Sham equations is to use some known basis set to expand the sought one-electron wave-functions $\psi_{\mathrm{i}}$. That is to solve the Kohn-Sham system of equations

we make an expansion

$$
\left[-\frac{\hbar^{2}}{2 m} \nabla^{2}+\mathrm{U}\right] \psi_{\mathrm{j}}=\varepsilon \psi_{\mathrm{j}}
$$

$$
\psi_{\mathrm{j}}=\sum_{i} c_{i} \varphi_{i},
$$

where $\varphi_{i}$ are some known basis functions (for example plane waves) and $c_{i}$ are unknown coefficients, which have to be found.

Then the Kohn-Sham equations looks like:

$$
\left[-\frac{\hbar^{2}}{2 m} \nabla^{2}+\mathrm{U}\right] \sum_{i} c_{i} \varphi_{i}=\varepsilon \sum_{i} c_{i} \varphi_{i}
$$

If we multiply every equation sequentially with all the $\varphi_{\mathrm{j}}{ }^{*}$ and integrate the left- and right-hand sides of the equations over the whole space, we end up with the system of equations, which can shortly be written as

$$
|\mathrm{H}-\varepsilon \mathrm{O}| \mathrm{C}=0 \text {, }
$$

where $\mathrm{C}$ is the column of unknown coefficients (see Eq. (2.10))

$$
\mathrm{C}=\left(\begin{array}{c}
c_{1} \\
\cdots \\
c_{n}
\end{array}\right)
$$

and $\varepsilon$ will be the set of energy eigenvalues. $\mathrm{H}$ and $\mathrm{O}$ are the so-called Hamiltonian and overlap matrices, which in Dirac bracket notation are given as

$$
\begin{aligned}
\mathrm{H}_{\mathrm{ij}} & =\left\langle\varphi_{j}\left|-\frac{\hbar^{2}}{2 m} \nabla^{2}+\mathrm{U}\right| \varphi_{i}\right\rangle \\
\mathrm{O}_{\mathrm{ij}} & =\left\langle\varphi_{i} \mid \varphi_{j}\right\rangle
\end{aligned}
$$

To find a nontrivial solution of Eq.(2.12), the necessary condition is to nullify the determinant (see Eq. (2.10)):

$$
\operatorname{det}|\mathrm{H}-\varepsilon \mathrm{O}|=0
$$


From solving Eq. (2.14) all the eigenvalues $\varepsilon_{i}$ can be extracted and then $C$ can be evaluated. The solution of the Kohn - Sham system of equations without electron-electron interaction gives the orbitals $\psi_{\mathrm{i}}$, from which the electron density $\mathrm{n}(\mathrm{r})$ (see Eq. (2.8)) of the original many-particle system is restored and the self-consistent procedure described above can be done.

\section{Exchange-correlation functional}

Exact analytical expressions for the functionals of the exchange and correlation energy are known only for the special case of a gas of free electrons. However, existing approximations allow us to calculate a number of physical quantities with sufficient accuracy [13].

The most common are the local density approximation (LDA), in which it is assumed that the functional calculated for a certain point in space depends only on the density at this point [14]:

$$
E_{x c}^{L D A}=\int \varepsilon_{x c}(n) n(r) d^{3} r
$$

$\varepsilon_{\mathrm{xc}}[\mathrm{n}(\mathrm{r})]$ is the exchange-correlation energy density; and the generalized gradient approximation (GGA) method [15]-[17], it is also local, but, unlike the local density method, takes into account the density gradient at the point of consideration:

$$
E_{x c}^{G G A}=\int \varepsilon_{x c}[n(r), \nabla n] n(r) d^{3} r
$$

For the GGA method, there are a number of successful parameterizations that allow one to increase approximation accuracy, for example, PBE [18].

\section{The Exact Muffin-Tin Orbitals method (EMTO)}

As already discussed above, to effectively solve the system of KohnSham equations, it is necessary to use a basis set of wave-functions. This basis may be very different. For example, in this work we used mostly the plane wave basis (in the Projector Augmented Wave Technique (PAW) ) or so-called exact muffin-tin orbitals (in the Exact Muffin-Tin Orbital (EMTO) method).

The exact self-consistent solution of the single-electron Kohn-Sham equations for overlapping spherical Muffin-Tin potentials (MT) allows us to achieve accuracy comparable to methods with full non-spherical 
potential, while maintaining the effectiveness of methods based on the use of MT potentials.

EMTO allows to calculate exactly the one-electron total energy for optimized overlapping MT potentials by using a Green's function formalism. EMTO method is different to the methods assuming nooverlapping MT potentials. It takes large, overlapping potential spheres that provide an accurate representation of the exact one-electron potential. The EMTO therefore combines efficiency of standard MT methods, but does not suffer from the shape approximations employed for the potential and density. In particular, non-spherically symmetric full charge density can be calculated.

EMTO is nicely combined with coherent potential approximation (CPA). CPA is the best single-site approach for alloy modeling. An effective medium is constructed in such a way that it corresponds to a simulated disordered alloy on average, so an atom fills the other atoms as a mean field. This condition is provided by a self-consistent solution of the CPA system of equations [19], [20].

\section{Projector Augmented Wave method (PAW)}

In modern calculations, the projector augmented wave method (PAW) [21] is widely used. This method combines the simplicity of a firstprinciples pseudopotential method and the accuracy of all-electron methods. The main idea of the PAW method is to conduct a linear transformation of pseudo-wave functions into exact, complete oneelectron Kohn-Sham functions. The wave functions of an atom in a material are divided by an additional construction - a sphere that separates valence electrons from core electrons. The wave functions inside the sphere are represented as partial functions, and the wave functions outside the sphere are described by plane waves [21].

The nucleus and electrons in deep in energy so-called core orbitals are replaced by a pseudopotential. All electron wave that strongly oscillates in the core region, is replaced by a smooth function, which is easily expanded in plane waves. All-electron valence functions $\psi_{k}$ are linearly transformed into the smooth functions with help of projector operators. The latter are defined so that they connect the smooth pseudowave function $\tilde{\psi}$ with the real one by some linear projector operator $\tau$ :

$$
\psi_{k}=\tau \tilde{\psi}
$$

The full wavefunction in all space looks like 


$$
|\psi\rangle=|\tilde{\psi}\rangle+\sum_{m}\left(\left|\psi_{m}\right\rangle-\left|\tilde{\psi}_{m}\right\rangle\right)\left\langle\tilde{p}_{i} \mid \tilde{\psi}\right\rangle
$$

where $\tilde{p}_{i}$ is the set of corresponding projectors.

The actual projectors can be set in several ways, depending on the specifics of the task.

Description and interpretation of the method, as we use it, are given in Ref. [22].

\section{Molecular dynamic (MD)}

Most of the studies in materials science from first principles relate mainly to the properties of the ground state of elements and their alloys. However, the properties at high temperatures might be different. More than that, many high-temperature phases do not exist at low temperatures due to the presence of dynamic and/or mechanical instabilities. To be able to accurately consider such cases, a rather computationally expensive method of ab initio molecular dynamics (AIMD) has to be used.

A serious drawback of this approach is the large statistical error and the duration of calculation to obtain results with high accuracy. [23]. Modeling using molecular dynamics requires the determination of a potential function that describes the interaction of a particle in a simulation. Potentials are usually determined using molecular and classical mechanics, which describes the mechanical interactions of particles with particles and addresses structural changes. This method takes into account the thermal vibrations of ions; therefore, it is necessary for calculations at high temperature. Classical mechanics is used to describe the motion of atoms or particles. Potential functions $\mathrm{U}$ are formulated as the sum of the interactions between the particles of the system.

AIMD works as follows: forces are calculated from first principles (within DFT) for atoms in the initial positions, then, taking these forces into account, the atoms move to a new position according to the second Newton's law. Then a new MD step is done, and forces are calculated again. Then we repeat this procedure as many times as necessary. Usually the temperature is maintained with help of a thermostat. That is there is an exchange of heat of the calculated system and some heat bath, which provides scaling of atomic velocities and therefore the kinetic energy of the nuclei in a way that the required temperature is maintained on average. The temperature of the classical system of many bodies can be determined using the uniform distribution of energy over all degrees of freedom that quadratically enter the Hamiltonian of the system. 
Accordingly, we can connect the velocities and the average kinetic energy per a degree of freedom. Therefore the momentarily temperature is defined from velocities as

$$
T=\frac{2}{3 k_{B}}\left\langle\frac{1}{N} \sum_{i=1}^{N} \frac{m_{i} v_{i}^{2}}{2}\right\rangle,
$$

where $k_{B}$ is Boltzmann constant, $m_{i}$ the mass of atom $\mathrm{i}, v_{i}$ the velocity of atom i, and $N$ is the total number of atoms.

Thermostats, like the the Nosé-Hoover one [24]-[26], which we used, enforce the correct distribution of the total kinetic energy over time. Then the sets of configurations obtained in the course of calculations by the MD method are distributed in accordance with the Boltzmann distribution function. All the forces acting on the atoms are taken into account and for each new time step the equations of Newton's motion are integrated. The forces of interatomic interaction can be represented as the gradient of the potential energy of the system. Then the steps necessary for balancing the system are cut off and the data obtained for the system are averaged over time steps. Thus, we can obtain the timeaverages of the desired properties.

Atom configurations must be compatible with the design structure, and trajectories must be generated in accordance with thermodynamic conditions.

The coordinates $\mathrm{r}$ and velocities $\mathrm{v}$ of atoms are calculated using algorithms for integrating the equations of motion with given conditions, based on the Verlet scheme [27]:

$$
r(\mathrm{t}+\Delta \mathrm{t})=\mathrm{r}(\mathrm{t})+\Delta \mathrm{t} v\left(\mathrm{t}+\frac{1}{2} \Delta \mathrm{t}\right)
$$

where $r(t)$ is the position at time $t$ and $r(t+\Delta t)$ is new the position of the atom at the next MD-step at time $t+\Delta t$.

$$
\mathrm{v}\left(\mathrm{t}+\frac{1}{2} \Delta \mathrm{t}\right)=\mathrm{v}\left(\mathrm{t}-\frac{1}{2} \Delta \mathrm{t}\right)+\Delta \mathrm{t} \frac{F j(t)}{m j}
$$

where $F_{j}$ is the force acting on atom $j$.

$$
\mathrm{F}_{\mathrm{j}}=-\frac{1}{r_{i j}}\left[\frac{\partial}{\partial r_{i j}} U\left(r_{i j}\right)\right] r_{i j}
$$

$\mathrm{U}$ is the potential energy. 


\section{Special Quasirandom Structures (SQS)}

Treating alloys we need to deal with a configurational disorder present in solid solutions. The most straightforward approach consists of employing the so-called supercells, the proper concentration of the alloy components is reached by adjusting the size of the supercell. The resulting supercell contains $n \times m \times k$ unit cells, where $n, m$, and $k$ are integer numbers. The supercell, which contains several unit-cells, is than converted using the periodic boundary conditions. So, the "randomness" of the random alloy depends on the particular atom placing. Depending on the configuration, different values of the total energy or other properties may be obtained. This problem can be solved, for example, using the Special Quasirandom Structures (SQS) method [28], [29]. It allows one to perform simulations of the disordered alloy modelled by a quasi-random, but still ordered supercell, enabling the straightforward use of a method like PAW. The SQS is constructed in such a way as to ensure that the short-range order parameters for several neighboring coordination shells are as close to zero on the average as possible, similar to their values in the case of complete disorder [28]. Consequently, the treatment of the disordered material is technically the same as in the standard electronic structure calculations of ordered compounds, e.g. at the same level of accuracy. In particular, the full non-spherical shape of the crystal potential can be treated explicitly, which makes it possible to carry out the full relaxation of atomic positions in the supercell. Unfortunately, the supercells often contain several dozens of atoms, which greatly (as $\mathrm{N}^{3}$, where $\mathrm{N}$ is number of atoms in the SQS) increases the costs of such calculations in comparison to those for ordered compounds. 

Chapter 3

\section{Elastic Properties: general theory}

\section{Single-crystal elastic constants}

The stress-strain relations can be obtained in the form of Hooke's law

$$
\sigma_{i}=\sum_{j=1}^{6} C_{i j} \varepsilon_{j},
$$

where ${ }^{\sigma_{i}},{ }^{\varepsilon_{j}}$, and ${ }^{C_{i j}}$ represent the components of the stress, strain and stiffness tensor in Voigt notation, respectively. The matrix form of Eq. (3.1) in the case of cubic crystals is

$$
\left(\begin{array}{l}
\sigma_{1} \\
\sigma_{2} \\
\sigma_{3} \\
\sigma_{4} \\
\sigma_{5} \\
\sigma_{6}
\end{array}\right)=\left(\begin{array}{cccccc}
C_{11} & C_{12} & C_{12} & 0 & 0 & 0 \\
C_{12} & C_{11} & C_{12} & 0 & 0 & 0 \\
C_{12} & C_{12} & C_{11} & 0 & 0 & 0 \\
0 & 0 & 0 & C_{44} & 0 & 0 \\
0 & 0 & 0 & 0 & C_{44} & 0 \\
0 & 0 & 0 & 0 & 0 & C_{44}
\end{array}\right)\left(\begin{array}{c}
\varepsilon_{1} \\
\varepsilon_{2} \\
\varepsilon_{3} \\
\varepsilon_{4} \\
\varepsilon_{5} \\
\varepsilon_{6}
\end{array}\right)
$$

To obtain the elastic constants the following non-volume-conserving distortion were applied in this work:

$$
\varepsilon(\delta)=\left(\begin{array}{l}
\delta \\
0 \\
0 \\
0 \\
0 \\
\delta
\end{array}\right),
$$

In Eq. (3.3) $\delta$ is the magnitude of the deformation, which we varied from -0.02 to 0.02 in steps of 0.01 .

Obviously, Eq. (3.3) gives the deformation matrix: 
Chapter 3

$$
1+\varepsilon_{1}(\delta)=\left(\begin{array}{ccc}
1+\delta & \frac{\delta}{2} & 0 \\
\frac{\delta}{2} & 1 & 0 \\
0 & 0 & 1
\end{array}\right)
$$

which allows us to calculate directly all the necessary moduli.

The basis vectors of the distorted structure are:

$$
\mathrm{a}^{\prime}=\mathrm{a}(1+\delta, \delta / 2,0), \mathrm{b}^{\prime}=\mathrm{a}(\delta / 2,1,0), \mathrm{c}^{\prime}=\mathrm{a}(0,0,1)
$$

The undistorted bcc titanium structure and distorted structure vectors with exaggerated $\delta=0.3$ are shown in Figure 3.1. This type of distortion slightly overestimates the lattice response to the applied deformation due to the presence of an additional term in the expansion of free energy associated with a change in volume, but it allows us to calculate all the elastic cubic crystal constants at once:

$$
C_{11}(T)=\frac{d \overline{\sigma_{1}}(T)}{d \delta}, C_{12}(T)=\frac{d \overline{\sigma_{2}}(T)}{d \delta}, C_{44}(T)=\frac{d \overline{\sigma_{6}}(T)}{d \delta},
$$

where $\overline{\sigma_{\imath}}$ is the stress value averaged over the MD.

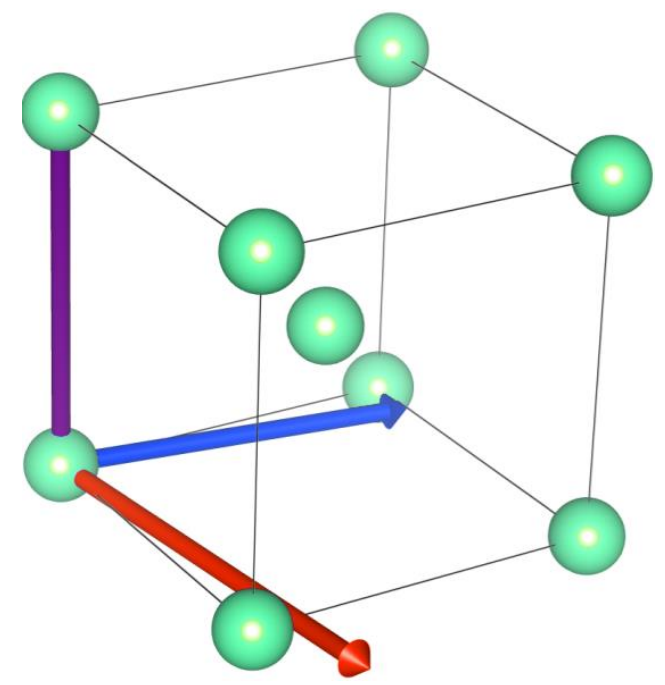

Fig. 3.1. Undistorted bec structure and primitive vectors of the distorted lattice according to the distortion in Eq. (3.3) : red - a', blue - b' and purple - c'. For illustration an exaggerated value of $\delta=0.3$ is used. 
We note that calculations of the tensorial quantities (like elastic moduli) by a supercell technique is less straightforward than for the scalar quantities (like total energies). Indeed, the SQS approach does not aim to generate structures that preserve the point group symmetry of an alloy and thus to provide the proper description of its tensorial properties. In almost all practical cases, the use of the SQS method leads to a disordered alloy models, which do not have the full cubic symmetry [30]. Therefore, it is necessary to use the projection technique introduced by Moakher and Norris [31] to derive the closest elastic tensors with cubic symmetry when modeling an alloy. In case of the SQS description of an alloy with a cubic symmetry, it reduces to performing calculations of $\mathrm{C}_{11}, \mathrm{C}_{12}$, and $\mathrm{C}_{44}$ with Eq. (3.6) for all permutations of the $\mathrm{a}, \mathrm{b}$, and $\mathrm{c}$ axis [30]. The resulting $\mathrm{C}_{11}, \mathrm{C}_{12}$, and $\mathrm{C}_{44}$ for the alloy are then obtained as the averages of those calculated from Eq. (3.6) for every permutation of the axis. This procedure ensures the correct symmetry of the elastic moduli and faster convergence of their calculations with respect to the size of the SQS.

The volume-conserving orthorhombic

$$
1+\varepsilon_{1}(\delta)=\left(\begin{array}{ccc}
1+\delta & 0 & 0 \\
0 & 1-\delta & 0 \\
0 & 0 & \frac{1}{1-\delta^{2}}
\end{array}\right)
$$

and monoclinic

$$
1+\varepsilon_{2}(\delta)=\left(\begin{array}{ccc}
1 & \delta & 0 \\
\delta & 1 & 0 \\
0 & 0 & \frac{1}{1-\delta^{2}}
\end{array}\right)
$$

strain matrices are applied to determine the elastic constants $\mathrm{C}^{\prime}$ and $\mathrm{C} 44$ of alloys by the EMTO-CPA method.

There elastic constants are obtained not from stresses, which are not calculated by EMTO-CPA, but from the internal energy response to the small distortions [19].

That is the change in total energy with respect to the undistorted structure in case of the distortion in Eq. (3.8) is

$$
\Delta \mathrm{E}=2 \mathrm{~V} \mathrm{C}^{\prime} \delta^{2}+\mathrm{O}\left(\delta^{4}\right)
$$

and in case of the distortion in Eq. (3.9) is 


$$
\Delta \mathrm{E}=2 \mathrm{~V} \mathrm{C}_{44} \delta^{2}+\mathrm{O}\left(\delta^{4}\right) .
$$

In Eqs. (3.10-3.11) V is the volume of the system.

The actual total energies were calculated for six distortions $(\delta=0.00$ $-0.05)$.

\section{Elastic moduli of polycrystalline alloys}

Usually materials used in applications are polycrystals, i.e. some aggregates of single crystals (grains). To describe their elastic moduli we need special assumptions. Under the assumption of randomly distributed orientations of grains macroscopical properties can be treated as isotropic. Then the ways to average monocrystalline elastic moduli are those, which are suitable for an isotropic body. Voigt introduced averaging of the matrix of elastic moduli of monocrystals, and then A. Reuss suggested averaging the matrix of compliances. These averages correspond to the assumptions about the uniform deformations in a polycrystal - in the first case, and about the uniform stresses - in the second one. R. Hill later showed that the calculated values of bulk and shear moduli of the polycrystal in these averages give upper and lower variational bounds of these properties, respectively. These boundaries can be quite wide in the case of a large anisotropy of the elastic properties of the polycrystal.

Let us consider these points in more detail.

We take the tensor of monocrystalline elastic moduli in an arbitrary coordinate system, averaged over the angles in an implicit form:

$$
\begin{gathered}
<B \geq \frac{1}{9} \sum\left(\lambda_{n}+6 \mu_{n}+4 v_{n}\right) \\
<G \geq \frac{1}{15} \sum\left(\lambda_{n}+10 v_{n}\right),
\end{gathered}
$$

where $\lambda_{n}, \mu_{n}$, and $v_{n}$ are so-called compliance coefficients, which can be given in terms of compliances (or, of course, elastic constants).

From here we find the averaged value of the Voigt bulk (B) and shear (G) moduli, which assumed the uniform deformation in all grains, for the cubic symmetry:

$$
<B \geq \frac{1}{3}\left(C_{11}+2 C_{12}\right),<G \geq \frac{1}{5}\left[\left(C_{11}-C_{12}\right)+3 C_{44}\right]
$$


As mentioned above, the method by Reuss is based on averaging the compliance tensor. The stress is assumed to be the same in all grains. As in the previous case, one can replace the averaging over the angles by calculating tensor convolutions:

$S_{i j k l}=\sum\left[\chi_{n} \delta_{i n} \delta_{j n} \delta_{k n} \delta_{l n}+\mu_{n}\left(\delta_{i n} \delta_{j n} \delta_{k l}+\delta_{i j} \delta_{k n} \delta_{l n}\right)+\right.$

$\left.v_{n}\left(\delta_{i n} \delta_{j k} \delta_{l n}+\delta_{j n} \delta_{i k} \delta_{l n}+\delta_{i n} \delta_{j l} \delta_{k n}+\delta_{j n} \delta_{i l} \delta_{k n}\right)\right]$,

where $\delta_{i j}$ is the Kronecker symbol, i.e. it is equal to 1 when $\mathrm{i}=\mathrm{j}$ and $\mathrm{o}$ otherwise.

The compliance coefficients $\lambda_{n}, \mu_{n}$, and $v_{n}$ are expressed as follows in terms of the components of the two-index compliance matrix:

$$
\begin{gathered}
\lambda_{1}=S_{11}+S_{23}+\frac{1}{2} S_{44}-\frac{1}{2}\left(2 S_{12}+2 S_{13}+S_{55}+S_{66}\right) \\
\lambda_{2}=S_{22}+S_{13}+\frac{1}{2} S_{55}-\frac{1}{2}\left(2 S_{12}+2 S_{23}+S_{44}+S_{66}\right) \\
\lambda_{3}=S_{33}+S_{12}+\frac{1}{2} S_{66}-\frac{1}{2}\left(2 S_{13}+2 S_{23}+S_{44}+S_{55}\right) \\
2 \mu_{1}=S_{12}+S_{13}-S_{23}, 8 v_{1}=S_{55}+S_{66}-S_{44} \\
2 \mu_{2}=S_{12}+S_{23}-S_{13}, 8 v_{2}=S_{44}+S_{66}-S_{55} \\
2 \mu_{3}=S_{13}+S_{23}-S_{12}, 8 v_{3}=S_{44}+S_{55}-S_{66}
\end{gathered}
$$

Now we average the compliance tensor from Eq. (3.15) and using equalities

$$
\frac{1}{\mathrm{~B}}=\mathrm{S}_{\mathrm{iikk}}, \quad \frac{1}{\mathrm{G}}=\frac{2}{5}\left(\mathrm{~s}_{\mathrm{ikik}}-\frac{1}{3} \mathrm{~S}_{\mathrm{iikk}}\right)
$$

we obtain:

$$
<\frac{1}{B} \geq \sum\left(\lambda_{n}+6 \mu_{n}+4 v_{n}\right), \quad<1 / G \geq \frac{4}{15} \sum\left(\lambda_{n}+10 v_{n}\right)
$$

Substituting the explicit values of the coefficients $\lambda_{n}, \mu_{n}$, and $v_{n}$ here, we find the average bulk and shear moduli of polycrystals. For cubic symmetry, taking into account the relations $S_{11}=S_{22}, S_{13}=S_{22}, S_{44}=S_{55}$, $\mathrm{S}_{11}=\mathrm{S}_{33}, \mathrm{~S}_{12}=\mathrm{S}_{13}, \mathrm{~S}_{44}=\mathrm{S}_{66}$, we obtain

$$
<1 / B \geq 3\left(S_{11}+2 S_{12}\right), \quad<1 / G \geq \frac{1}{5}\left[4\left(S_{11}-S_{12}\right)+3 S_{44}\right]
$$


Comparing the results of averaging the tensors of elastic constants and compliances, we notice that two different types of averaging lead to different results. An exception is the bulk modulus of polycrystals of cubic symmetry, which, by virtue of the relation:

$$
C_{11}+2 C_{12}=\left(S_{11}+S_{12}\right)^{-1}
$$

that holds for crystals of this symmetry, turns out to be isotropic.

Denoting the elastic moduli obtained by averaging tensor $\mathrm{C}$ by $\mathrm{B}_{\mathrm{V}}$ and $\mathrm{G}_{\mathrm{V}}$, and by averaging tensor $\mathrm{S}$ by $\mathrm{B}_{\mathrm{R}}$ and $\mathrm{G}_{\mathrm{R}}$ (of course, "V" points to Voigt and " $R$ " to Reuss), we can write the following inequality:

$$
B_{R} \leq \mathrm{B}^{*} \leq B_{V}, \quad G_{R} \leq \mathrm{G}^{*} \leq G_{V},
$$

where $\mathrm{B}^{*}$ and $\mathrm{G}^{*}$ are the effective elastic moduli connecting the averaged stresses and deformations.

In some cases, the arithmetic average of the values found by averaging using Voigt and Reuss approaches leads to good results.

We denote the macroscopic stress and strain in the polycrystal $\mathrm{S}$ and $\mathrm{E}$; for convenience, $\mathrm{S}$ and $\mathrm{E}$ are vectors in $9 \mathrm{~d}$ space. We denote $\sigma$ and $\varepsilon$ by macroscopic stresses and deformation at any point in any grain of the crystal. If a crystal contains a significantly large number of grains and is macroscopically homogeneous, then, according to Bishop and Hill,

$$
S . E=\int \sigma \cdot \varepsilon d V
$$

In terms of the Maxwell relation on reciprocity of displacements, the following identities are true at each point of the polycrystal:

$$
\begin{gathered}
\sigma . \varepsilon+\left(\sigma-\sigma^{*}\right) \cdot(\varepsilon-E) \equiv \sigma^{*} \cdot E+2(\varepsilon-E) \cdot \sigma . \\
\sigma . \varepsilon+(\sigma-\mathrm{S}) \cdot\left(\varepsilon-\varepsilon^{*}\right) \equiv \mathrm{S} \cdot \varepsilon^{*}+2(\sigma-\mathrm{S}) \cdot \varepsilon,
\end{gathered}
$$

where $\sigma^{*}$ is the stress that could exist in a crystal with a local orientation and have a strain $\mathrm{E} ; \varepsilon^{*}$ is the strain that could be generated in such a crystal by means of stress S. Since the scalar product of any associated stress and strain is positive (equal to two energy densities)

$$
\begin{gathered}
\sigma . \varepsilon \leq \sigma^{*} \cdot E+2(\varepsilon-E) . \sigma \\
\sigma . \varepsilon \leq S . \varepsilon^{*}+2(\sigma-S) . \varepsilon .
\end{gathered}
$$


Bishop and Hill showed that the volume integral of the last term in each inequality can be reduced. Therefore, from Eq. (3.21)

$$
S . E \leq\left\{\begin{array}{l}
E \cdot \int \sigma^{*} d V \\
S . \int \varepsilon^{*} d V
\end{array}\right.
$$

The left-hand side of this equation is equal to two actual energy densities (which are conditionally determined in the form of macroscopic quantities); the right-hand side of this equation is equal to two actual energy densities, which could be calculated using the Voigt and Reuss theories, respectively.

For a polycrystal, which is macroscopically isotropic, Eq. (3.24) should lead to the following symmetry and automatically square expressions in the principal components of stress and strain:

$$
\left\{\begin{array}{l}
\mathrm{B}\left(\mathrm{E}_{1}+\mathrm{E}_{2}+\mathrm{E}_{3}\right)^{2}+\frac{2}{3} \mathrm{G} \Sigma\left(\mathrm{E}_{1}-\mathrm{E}_{2}\right)^{2} \leq \mathrm{B}_{\mathrm{V}}\left(\mathrm{E}_{1}+\mathrm{E}_{2}+\mathrm{E}_{3}\right)^{2}+\frac{2}{3} \mathrm{G}_{\mathrm{V}} \Sigma\left(\mathrm{E}_{1}-\mathrm{E}_{2}\right)^{2} \\
\frac{1}{\mathrm{~B}}\left(\mathrm{~S}_{1}+\mathrm{S}_{2}+\mathrm{S}_{3}\right)^{2}+\frac{3}{2 \mathrm{G}} \Sigma\left(\mathrm{S}_{1}-\mathrm{S}_{2}\right)^{2} \leq \frac{1}{\mathrm{~B}_{\mathrm{R}}}\left(\mathrm{S}_{1}+\mathrm{S}_{2}+\mathrm{S}_{3}\right)^{2}+\frac{3}{2 \mathrm{G}_{\mathrm{R}}} \Sigma\left(\mathrm{S}_{1}-\mathrm{S}_{2}\right)^{2}
\end{array}\right.
$$

$\mathrm{B}$ and $\mathrm{G}$ are the actual volume and shear moduli; the indices $\mathrm{V}$ and $\mathrm{R}$ again mean the values calculated according to the Voigt and Reuss theories from the estimate of the integrals (3.24). Obviously, averaging the energy density is equivalent to averaging the stress-strain relationships for given stresses and strains. Due to the fact that equations (3.25) are valid for any stresses and strains, it follows:

$$
\mathrm{B}_{\mathrm{R}} \leq \mathrm{B} \leq \mathrm{B}_{\mathrm{V}} ; \mathrm{G}_{\mathrm{R}} \leq \mathrm{G} \leq \mathrm{G}_{\mathrm{V}}
$$

As can be easily seen, Eq. (3.26) is equivalent to Eq. (3.20).

Corresponding calculations of the Poisson's ratio and Young's modulus are obtained by replacing the appropriate values of B and $\mathrm{G}$

$$
\begin{gathered}
v=\frac{1}{2}\left[1-\frac{3 \mathrm{G}}{3 \mathrm{~B}+\mathrm{G}}\right], \quad \frac{1}{\mathrm{E}}=\frac{1}{3 \mathrm{G}}+\frac{1}{9 \mathrm{~B}} \\
E_{R} \leq E \leq E_{V}
\end{gathered}
$$

Thus, it shows that the Voigt moduli exceed the Reuss modules, along with the fact that the true values lie between them. The previous conclusion is completely mathematical, but it depends on the accuracy of the statistical hypotheses inherent in Eq. (3.21).

Voigt showed that 
Chapter 3

$$
\left\{\begin{array}{c}
9 B_{V}=\left(C_{11}+C_{22}+C_{33}\right)+2\left(C_{12}+C_{23}+C_{31}\right) \\
15 G_{V}=\left(C_{11}+C_{22}+C_{33}\right)-\left(C_{12}+C_{23}+C_{31}\right)+3\left(C_{44}+C_{55}+C_{66}\right)
\end{array}\right.
$$

Reusss showed that

$$
\left\{\begin{array}{c}
1 / B_{R}=\left(S_{11}+S_{22}+S_{33}\right)+2\left(S_{12}+S_{23}+S_{31}\right) \\
15 / G_{R}=4\left(S_{11}+S_{22}+S_{33}\right)-4\left(S_{12}+S_{23}+S_{31}\right)+3\left(S_{44}+S_{55}+S_{66}\right)
\end{array}\right.
$$

It can be noted that only 9 out of 21 independent single crystal constants are found in these formulas for macroscopic modules.

In particular, for a cubic crsystals

$$
C_{11}=C_{22}=C_{33}, \quad C_{12}=C_{23}=C_{31}, \quad C_{44}=C_{55}=C_{66}
$$

with similar ratios for $\mathrm{S}$ coefficients when stress and strain components are considered in cubic axes:

$$
C_{11}-C_{12}=\frac{1}{S_{11}-S_{12}}, \quad C_{11}+2 C_{12}=\frac{1}{S_{11}+2 S_{12}}, C_{44}=\frac{1}{S_{44}}
$$

Therefore,

$$
\left\{\begin{array}{c}
\mathrm{B}_{\mathrm{R}}=\mathrm{B}_{\mathrm{V}}=\oint\left(\mathrm{C}_{11}+2 \mathrm{C}_{12}\right) \\
5 \mathrm{G}_{\mathrm{V}}=\left(\mathrm{C}_{11}-\mathrm{C}_{12}\right)+3 \mathrm{C}_{44}, \quad 5 / \mathrm{G}_{\mathrm{R}}=4\left(\mathrm{~S}_{11}-\mathrm{S}_{12}\right)+\mathrm{S}_{44} \\
\mathrm{~B}_{\mathrm{R}}=\mathrm{B}_{\mathrm{V}} ;
\end{array}\right.
$$

Otherwise, the uniform distributions of hydrostatic stress and strain in the polycrystal are comparable due to the fact that hydrostatic stress generates only anisotropic volume changes in the cubic crystal.

We can notice that using the Cauchy relation $\left(\mathrm{C}_{12}=\mathrm{C}_{44}\right)$ we can get $\mathrm{G}_{\mathrm{v}}=3 \mathrm{~B} / 5$ and $v_{V}=1 / 4$. From the isotropy condition, $\mathrm{C}_{11}-\mathrm{C}_{12}=2 \mathrm{C}_{44}$, we obtain $G_{R}=G_{V}$, which is expected due to the fact that the polycrystal is microscopically homogeneous. For small anisotropy values, the difference between $G_{R}$ and $G_{V}$ is a second order quantity:

$$
\mathrm{G}_{\mathrm{v}}-\mathrm{G}_{\mathrm{R}}=\frac{2}{5} \frac{\left[2 \mathrm{C}_{44}-\left(\mathrm{C}_{11}-\mathrm{C}_{12}\right)\right]^{2}}{\left[4 \mathrm{C}_{44}+3\left(\mathrm{C}_{11}-\mathrm{C}_{12}\right)\right]}
$$

Thus, the approximation closest to the experiment is the Hill approximation, which is suggested taking the average of the Voigt 
approximation (the same strain of all grains) and the Reuss one (the same stress in all grains), in the form:

$$
1 / 2\left(G_{R}+G_{v}\right) \text { or } \sqrt{(G r G v)}
$$

Two parts in Eq. (3.32) are usually reffered to as "arithmetic" and "geometric" Hill's average. Most common is the usage of the arithmetic one.

Thus, the elastic properties of a polycrystal can be calculated by knowing the elastic constants of a single crystal based on the aforementioned averaging, namely, Voigt, Reuss, and Hill.

The bulk modulus in the (arithmetic) Hill approximation $\mathrm{B}_{\mathrm{H}}$ is determined from the relation:

$$
B_{H}=\frac{B_{R}+B_{V}}{2}
$$

For cubic crystals the bulk moduli $\mathrm{B}_{\mathrm{R}}$ and $\mathrm{B}_{\mathrm{V}}$ in the Reuss and Voigt approximations, respectively, are equal:

$$
B_{R}=B_{V}=\frac{\left(C_{11}+2 C_{12}\right)}{3}
$$

The shear modulus in the Hill approximation $\mathrm{G}_{\mathrm{H}}$ is calculated by analogy from the relation:

$$
G_{H}=\frac{G_{R}+G_{V}}{2}
$$

where, as above, $\mathrm{G}_{\mathrm{R}}$ is the shear modulus in the Reuss approximation and $\mathrm{G}_{\mathrm{V}}$ is the shear modulus in the Voigt approximation, which can be written for cubic crystals as:

$$
\begin{gathered}
G_{R}=\frac{5 C_{44}\left(C_{11}-C_{12}\right)}{4 C_{44}+3\left(C_{11}-C_{12}\right)} \\
G_{V}=\frac{C_{11}-C_{12}+3 C_{44}}{5}
\end{gathered}
$$

Knowing B and G, we calculate the Young's modulus E and the Poisson's ratio $v$ from the relations:

$$
E=\frac{9 B G}{3 B+G}
$$




$$
v=\frac{3 B-2 G}{2(3 B+G)}
$$

The phenomenological criteria, which are quite often used in practice to determine whether a material is expected to be brittle or ductile, are those relying on the Cauchy pressure $\mathrm{P}_{\mathrm{c}}$ [32] or on the Pugh ratio $\mathrm{k}$ [33]. They are calculated as:

$$
\begin{gathered}
\mathrm{P}_{\mathrm{c}}=\left(\mathrm{C}_{12}-\mathrm{C}_{44}\right) \\
\mathrm{k}=\frac{G}{B}
\end{gathered}
$$

Derivations above are done based on Refs. [34]-[41]

\section{Calculations of elastic properties of Ti and its alloys}

\section{Details of EMTO-CPA calculations}

The calculation parameters were as follows. The basic set of EMTO included s-, p-, d- , and f - orbitals. The full charge density (FCD) [42] was represented by a single-centre expansion of the electron wave functions in terms of spherical harmonics with orbital angular moments $l_{F C D}^{\max }=8$. The integration in the irreducible part of the Brillouin zone was performed over a 29x29x29 grid of k points. The energy integration was carried out in the complex plane using a semielliptic contour comprising 24 energy points. The convergence of energy in the self-consistent run was $10^{-8}$ Ry.

\section{Details of PAW-SQS calculations}

The PAW method [21] was used within the framework of the density functional theory, as it implemented in the Vienna Ab Initio Software Package (VASP) [43], [44].

The SQSs for bcc Ti-V and Ni-Al-Ti alloys were constructed on a 128atom supercell consisting of $4 \times 4 \times 4$ simple cubic cells. To determine the numerical parameters of elastic constant calculations, a number of convergence tests were performed. To achieve accurate stresses, the cutoff energy had to be set to $460 \mathrm{eV}$ for the TiV system and $500 \mathrm{eV}$ for the Ni-Al-Ti system. The integration over the Brillouin zone was performed using a set of $3 \times 3 \times 3$ and $2 \times 2 \times 2$ k-points, respectively. 


\section{Details of MD calculations}

AIMD simulations were applied to calculate the temperature dependent elastic constants of pure bcc Ti. The effects of anharmonic lattice vibrations due to finite temperature on the stability of pure bcc $\mathrm{Ti}$ and Ti-V alloy were studied by means of density-functional theory calculations within the Projector Augmented Wave method as implemented in the Vienna Ab initio Simulation Package [43], [44]. The exchange-correlation energy was calculated within the generalized gradient approximation (GGA). $300 \mathrm{eV}$ plane-wave cutoff was used for pure bcc Ti and Ti-V alloy. The Brillouin zone integration was performed using a set of $2 \times 2 \times 2 \mathrm{k}$-points. The bcc structure of pure Ti and TiV alloy was described with a 128-atom supercell constructed as a $4 \times 4 \times 4$ simplecubic supercell, the same way as for static calculations described above.

These parameters were tested to be sufficient for convergence. To calculate the derivatives of stresses with respect to distortions at each temperature, the dependence of $\bar{\sigma}_{\imath}$ was fitted with a linear dependence by the least squares method. The error in determining the slope of the straight line (i.e. the elastic constants) characterizes both the error in determining the average values $\overline{\sigma_{l}}$ and possible deviations from the linear strain-stress dependence (i.e. Hooke's law).

\section{Results}

\section{Ti-V}

Using the EMTO-CPA and PAW-SQS methods, the dependences of single-crystal elastic constants and the polycrystalline elastic moduli for $\mathrm{Ti}-\mathrm{V}$ alloys on the concentration of vanadium in the range from $\mathrm{o}$ to 100 at.\% of V were found.

In Fig. 3.2. the calculated dependencies of the elastic constants $\mathrm{C}_{11}, \mathrm{C}_{12}$, and $\mathrm{C}_{44}$ are displayed. The moduli $\mathrm{C}_{11}$ and $\mathrm{C}_{12}$ obtained by the PAW-SQS and EMTO-CPA methods for the bcc Ti-V alloys increase monotonously with increasing vanadium concentration in the range from o to 100 at. \% of $\mathrm{V}$. The experimental data are presented in the range 30-100 at. \% V and the increase is almost linear in the whole concentration range. The obtained PAW-SQS results for $\mathrm{C}_{11}$ show good quantitative agreement with the experimental data, as well as good agreement with the data obtained on the ordered structure [45]. EMTO-CPA calculations predict somewhat higher values of $\mathrm{C}_{11}$. At the same time, the concentration dependence of $\mathrm{C}_{11}$ obtained in EMTO-CPA calculations is linear and it is in somewhat better agreement with experimental concentration dependence than the one predicted by PAW-SQS calculations. One 
reason for the kinks that we see in PAW-SQS calculations of $\mathrm{C}_{11}$ could be incomplete k-point convergence. Indeed, it is much easier to fully converge the EMTO-CPA calculations, as they are done for one (effective) atom per cell rather than PAW, while SQS calculations are done for a supercell with 128 atoms. In this sense, the efficiency of the EMTO-CPA method turns out to be beneficial for the accuracy of the trends, predicted with this method, even though the absolute values obtained with PAW-SQS calculations are in better agreement with experiment. Simultaneously, we see very good agreement between $\mathrm{C}_{12}$ elastic modulus obtained by the PAW-SQS and EMTO-CPA methods. In this case, the agreement with experiment is similar for both methods. Again, the latter predicts a smoother concentration dependence than the former, most probably because of the better numerical convergence. In summary, the results of the calculations obtained by the PAW-SQS and EMTO-CPA methods correctly reproduce the trends and are in reasonable agreement with each other and with experimental data [46], [47]. The largest deviation is observed in the vanadium-rich region, particularly with regard to the elastic constant $\mathrm{C}_{11}$. A poor reproducibility of the experimental data on $\mathrm{C}_{11}$ for pure vanadium is a known problem, and it has been pointed out in a number of theoretical papers by other authors [9], [48]-[52].

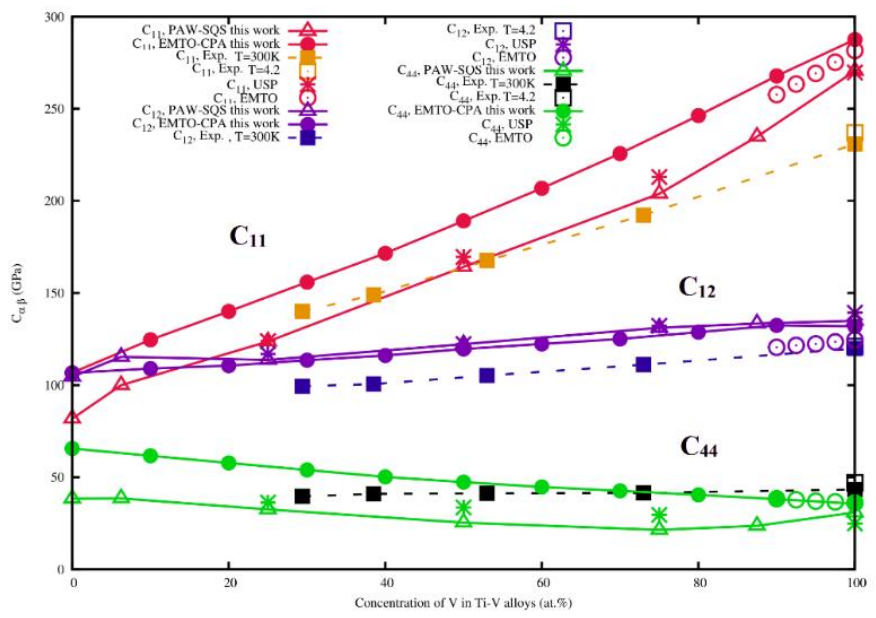

Fig.3.2. Dependence of elastic constants $\mathrm{C}_{11}, \mathrm{C}_{12}$, and $\mathrm{C}_{44}$ on $\mathrm{V}$ concentration in bcc Ti-V alloy. Red symbols correspond to the calculated values of $\mathrm{C}_{11}$. The calculated values of $\mathrm{C}_{12}$ are shown as the purple symbols. Green symbols are the calculated values of $\mathrm{C}_{44}$. The gold dashed lines with filled square symbols denote the experimental values of $C_{11}$ Ref.[46], the experimental values of $\mathrm{C}_{11}$ are shown as the gold empty square symbols (Ref. [47]). Blue symbols correspond to the experimental values of $\mathrm{C}_{12}$ 
(Refs. [46], [47]). Black symbols are experimental values of $\mathrm{C}_{44}$ from Refs. [46], [47]. Solid lines with filled circles are the data obtained in this work by the EMTO-CPA method. The triangular symbols are the data obtained in this work by the PAW-SQS method. The values of Ref. [45] are marked with the asterisks symbols. The values of EMTO calculations from Ref.

[48] are designated by the empty circular symbols.

Let us now discuss the elastic modulus $\mathrm{C}_{44}$. The experimental data measured for bcc Ti-V alloys indicate that the value of $\mathrm{C}_{44}$ is practically independent of the concentration and lies in the range from 40 to 43 GPa. It should be noted that the elastic constant $\mathrm{C}_{44}$ obtained in the EMTO-CPA calculations is monotonously decreasing with increasing V concentration. The curve obtained by the PAW-SQS method is not monotonous with a shallow minimum at 75 at. \% of V. The values obtained by the PAW-SQS method vary from 39 to $21 \mathrm{GPa}$ at vanadium concentrations from o to $100 \%$.

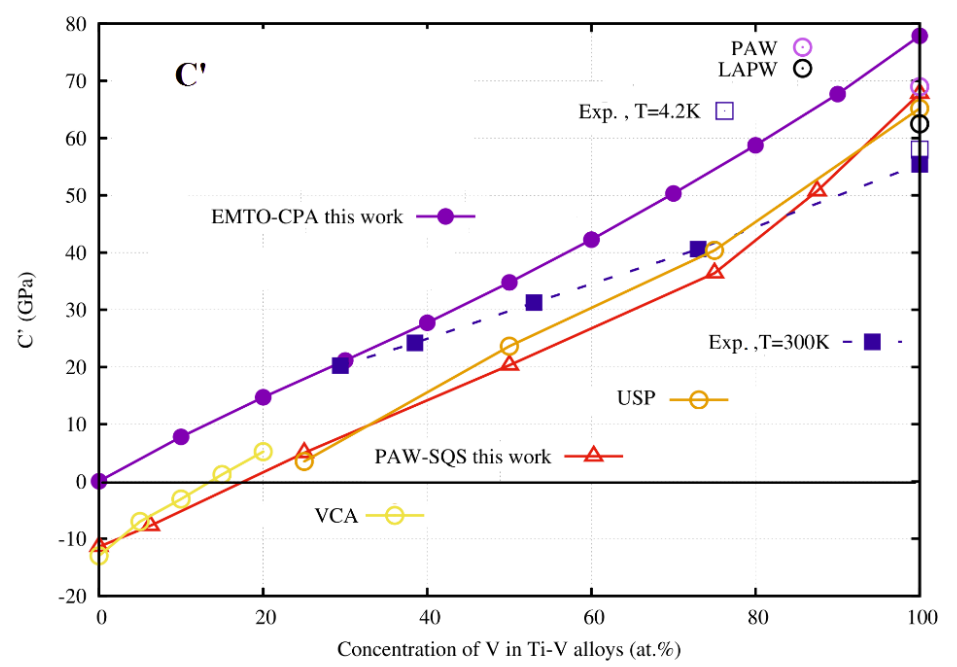

Fig. 3.3. Dependence of the elastic constant $\mathrm{C}^{\prime}$ on $\mathrm{V}$ concentration. Red triangular symbols denote the values obtained by the PAW-SQS method in this work. Purple filled circles are the values obtained by us with the EMTO-CPA method. The blue dashed lines with filled square symbols correspond the experimental data from Ref. [46]. The experimental data from Ref. [47] are designated by blue empty square symbols. Gold color shows results of Ref. [45]. The values of VCA calculations reported in Ref. [53] are marked with the yellow symbols. Purple empty circles show PAW values of Ref. [9]. The LAPW values of Ref. [49] are indicated by the black symbols. 
Figure 3.3 shows the dependence of the elastic constant $\mathrm{C}^{\prime}$ on the concentration $\mathrm{V}$ in $\mathrm{Ti}-\mathrm{V}$ alloys. Elastic constant $\mathrm{C}^{\prime}$ obtained by both methods increases monotonously with increasing vanadium concentration. The concentration dependence obtained by the PAW-SQS method is weakly nonlinear. Once again, there is a strong reason to believe that this is a numerical rather than physical effect, as both, the EMTO-CPA method and experiment, indicate close to linear concentration dependence of $\mathrm{C}^{\prime}$. In fact, a softening of this elastic constant can be used to characterize the mechanical stability of an alloy. Here one sees that $C^{\prime}$ becomes harder with increasing vanadium concentration, indicating the increasing mechanical stability of the system. One can also see that $\mathrm{C}^{\prime}$ obtained by using the EMTO-CPA and PAW-SQS methods are in a reasonable agreement with the experimental values and with each other in the stability region of the solid solution.

On the other hand, there is an important qualitative difference between the results of PAW-SQS and EMTO-CPA calculations. According to the former, the $\beta$-phase of $\mathrm{Ti}$ becomes mechanically unstable at $\mathrm{V}$ concentrations below $\sim 20$ at. \%, where $\mathrm{C}^{\prime}$ becomes negative. This is in good agreement with the experimental data, as well as with earlier theoretical studies [46], [53], [54]. On the contrary, the mechanical instability of the alloys in Ti-rich region is not reproduced by the EMTO-CPA method. Indeed, $\mathrm{C}^{\prime}$ calculated by this technique is above $\mathrm{o}$ even at zero vanadium concentration. One therefore concludes that in critical regions of peculiarities associated with elastic constants the EMTO-CPA results must be verified with more accurate calculations.

Figure 3.4 shows the Young's modulus E, the bulk modulus B, the shear modulus $\mathrm{G}$, and the Cauchy pressure Pc as a function of the concentration $\mathrm{V}$ in the Ti-V alloy. One can see that $\mathrm{B}$ and Pc obtained by the PAW-SQS and EMTO-CPA methods increase almost linearly with increasing vanadium concentration. On the contrary, the dependences of $\mathrm{G}$ and $\mathrm{E}$ are strongly nonlinear. In addition, it should be noted that upon the approach to the area of mechanical instability the results obtained by the PAW-SQS method predict a sharp decrease of the Young's modulus. This fact can be important for a design of new alloys, e.g. for biomedical applications. In this case, the materials with relatively low values of Young's moduli close to the values of human tissue (10-60 GPa) are needed to ensure biomechanical compatibility and uniform load distribution, avoiding bone degradation. 

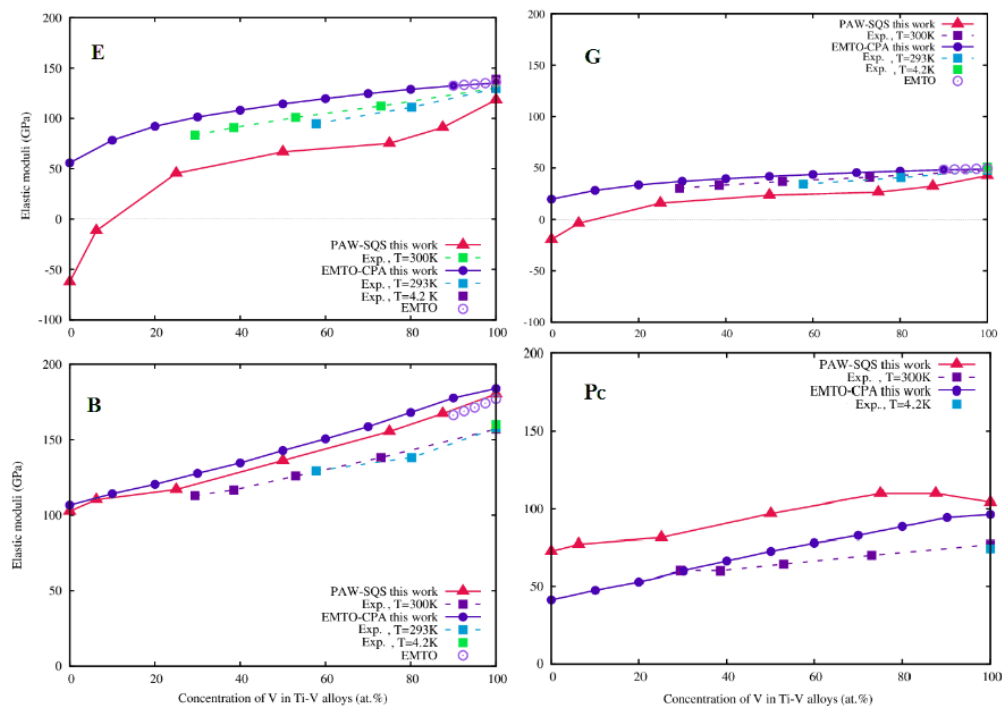

Fig. 3.4. Dependence of Young's modulus E, bulk modulus B, shear modulus G, and Cauchy pressure Pc on V concentration in the bcc Ti-V alloy. The experimental data are shown as dashed lines of blue, violet and green [46], [55], [47] colors. The data obtained by the PAW-SQS method in this paper are denoted by red triangles. The data obtained by the EMTO-CPA method are marked with blue filled circles. Purple empty circles display the values of EMTO-CPA calculations from Ref. [48].

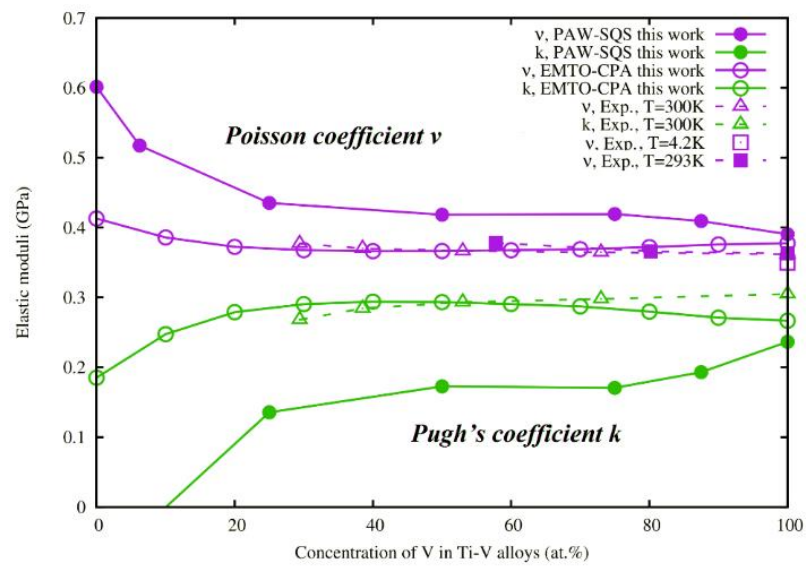

Fig. 3.5. Dependence of the Pugh's coefficient $k$ and the Poisson coefficient $v$ on Vanadium concentration in the bcc Ti-V alloy. Purple symbols denote values of the Poisson's ratio. The green symbols show the Pugh's coefficient values. Dashed lines with triangles and square symbols denote the 
experimental data. Filled circles show the data obtained by the PAW-SQS method in this paper. The data obtained by the EMTO-CPA method are shown as open circles.

Fig. 3.5 shows the dependences of the Pugh's coefficient $\mathrm{k}$ and the Poisson coefficient $v$ on the concentration $\mathrm{V}$ in the Ti-V alloy. The dependence of the coefficients on $\mathrm{V}$ concentration is quite weak in the region of mechanical stability of the bcc alloys, in agreement with experiment. On the other hand, it becomes quite strong for mechanically unstable alloys.

The investigation of the concentration dependences of the $\mathrm{G} / \mathrm{B}$ ratio and the Cauchy pressure $\mathrm{P}_{\mathrm{C}}$ of the bcc Ti-V alloys carried out by using the phenomenological correlations between the ductility and the elastic constants indicates a possible slight increase in ductility with increasing vanadium content. The monotonously increasing values of $P_{c}$ suggest that the fraction of the metallic component of the interatomic bond increases with the addition of vanadium ( $\left.\mathrm{C}_{12}>\mathrm{C} 44\right)$.

Using the EMTO and PAW methods, the dependences of elastic constants and the elastic moduli for Ti-V alloys on the concentration of vanadium in the range from $\mathrm{o}$ to 50 at. \% V were found.
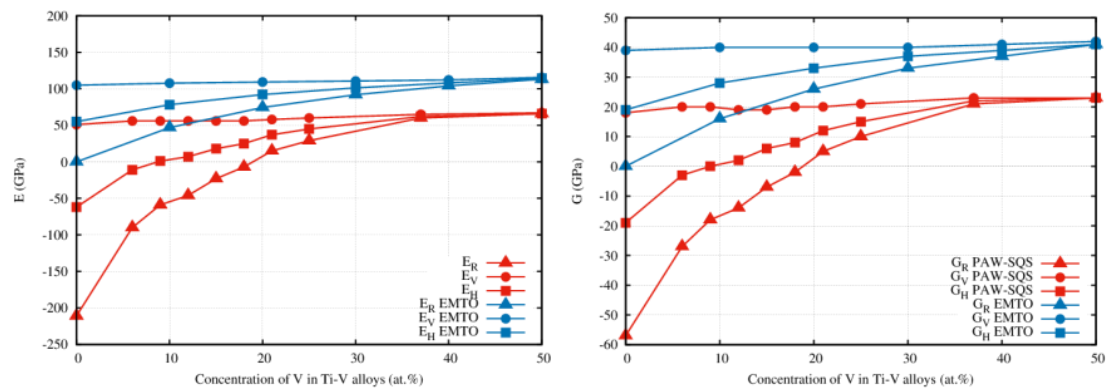

Fig. 3.6. Dependence of Young's modulus E and shear modulus G on V concentration in the bcc Ti-V alloy. The data obtained by the PAW-SQS method in this paper are denoted by red lines. The data obtained by the EMTO-CPA method are marked with blue lines. The solid lines with filled square symbols denote the Hill approximation. Solid lines with filled circles are the Voigt approximation. The triangular symbols are the Reuss approximation.

The elastic characteristics of polycrystalline materials are estimated by averaging the values obtained in the calculations of single crystals using the Voigt (V) -Reuss (R) -Hill (H) procedure. Figure 3.6 shows the results of such an assessment for Young's modulus and for shear modulus G. The data obtained by the PAW-SQS method in this paper are 
denoted by red lines. The data obtained by the EMTO-CPA method are marked with blue lines. The solid lines with filled square symbols denote the Hill approximation. Solid lines with filled circles are the Voigt approximation. The triangular symbols are the Reuss approximation.

Young's modulus E obtained by the both methods increases monotonously with increasing vanadium concentration. The modules $\mathrm{E}_{\mathrm{R}}, \mathrm{E}_{\mathrm{V}}$, and $\mathrm{E}_{\mathrm{H}}$ obtained by the EMTO methods for Ti-V alloys increase linearly with increasing vanadium concentration in the range from 10 to 50 at. \%. As can be seen in Figure 3.6; $\mathrm{E}_{\mathrm{V}}, \mathrm{E}_{\mathrm{H}}$, and $\mathrm{E}_{\mathrm{R}}$ approaching the area of instability, begin to diverge. The averages of Young's modulus diverge in the instability region. The concentration dependence obtained by the PAW-SQS method is characterized by weak nonlinearity.

The shear modulus G obtained by the methods of PAW and EMTO increases linearly with increasing vanadium concentration in the range from $\mathrm{o}$ to 50 at. \%. The dependences $\mathrm{G}\left(\mathrm{X}_{\mathrm{v}}\right)$ are weakly nonlinear. In the vicinity of the instability region, there is a significant discrepancy between the values of the elastic constants $\mathrm{G}_{\mathrm{H}}\left(\mathrm{X}_{\mathrm{v}}\right), \mathrm{G}_{\mathrm{R}}\left(\mathrm{X}_{\mathrm{V}}\right)$, and $\mathrm{G}_{\mathrm{V}}\left(\mathrm{X}_{\mathrm{V}}\right)$.

The divergence of different averaging schemes of $\mathrm{E}$ and $\mathrm{G}$ in the region of instability is related to different contribution of $C^{\prime}$, follows from Eqs. (3.31-3.37). Specifically, $\mathrm{G}_{\mathrm{R}}$ is proportional to $\mathrm{C}^{\prime}$ and goes to zero when C' does, different from $\mathrm{G}_{\mathrm{V}}$, which in this case becomes proportional to $\mathrm{C}_{44}$. As $\mathrm{C}_{44}$ increases with decreasing $\mathrm{V}$ concentration (see Fig. 3.2), the divergence between $G_{R}$ and $G_{V}$ at low vanadium concentrations, when $C^{\prime}$ becomes negative, is obvious from Eqs.(3.34-3.35). This propagates into E according to Eq.(3.36).

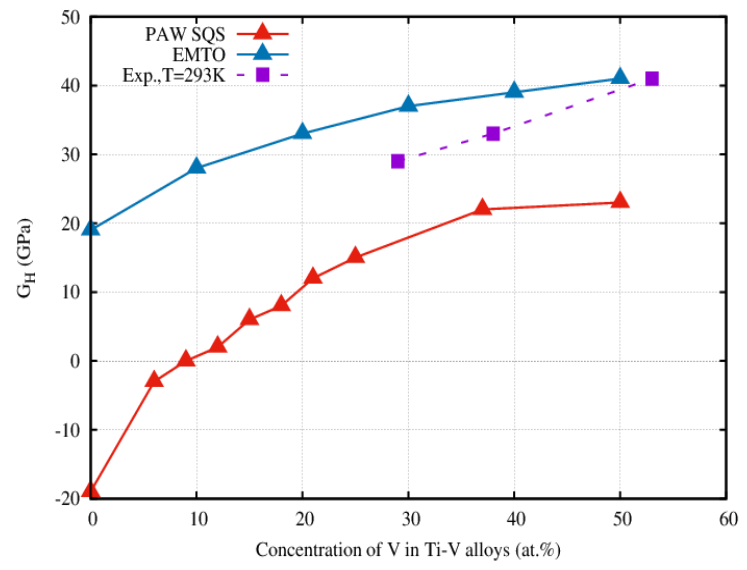

Fig. 3.7. Dependence of shear modulus $\mathrm{G}_{\mathrm{H}}$ on $\mathrm{V}$ concentration in the bcc Ti-V alloy. The purple square symbols with dashed lines denote experimental data. The data obtained by the PAW-SQS method in this paper are denoted by red 
triangles and solid line. The data obtained by the EMTO-CPA method are marked with blue filled triangles with solid line.

Figure 3.7 shows the dependence of shear modulus on the concentration of vanadium in Ti-V alloy. It is monotonous and $\mathrm{G}$ grows with the $\mathrm{V}$ concentration. Experimental data show the $\mathrm{G}$ growth in the whole addressed interval. Both theoretical dependences, from EMTO and PAW-SQS calculations, are nonlinear.

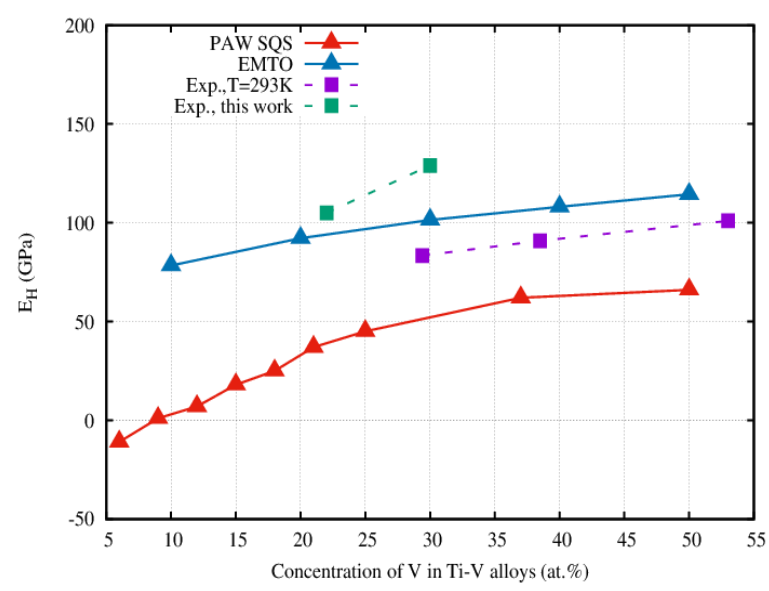

Fig. 3.8. Dependence of Young's modulus $\mathrm{E}_{\mathrm{H}}$ on $\mathrm{V}$ concentration in the bcc TiV alloy. The square symbols with dashed lines denote experimental date. The data obtained by the PAW-SQS method in this paper are denoted by red triangles. The data obtained by the EMTO-CPA method are marked with blue filled triangles.

The dependence of Young's modulus $\mathrm{E}_{\mathrm{H}}$ on the dopant content, obtained by the EMTO method, has a linear nature and shows good convergence with the experimental data in the stability region of the beta phase, however the instability region is not reproduced. The results obtained using the PAW method reflect the instability region. These results show that if one manages to decrease the vanadium concentration and to keep the bcc structure, better properties for biomedical materials can be obtained. Experimental data are presented in the range from $\sim 30$ to 50 at. \% of $\mathrm{V}$ and increase over the entire span.

In the experimental part of this work $\mathrm{Ti}-\mathrm{V}$ alloys were produced in thin films, which are close to the instability region (with the $\mathrm{V}$ concentration close to our predicted $\sim 20$ at. \%). They show a sharp change in the slope angle for the elastic modulus, which indicates the appearance of nonlinearity in the concentration dependence in the instability region. As can be seen from Figure 3.8, these experimental values lie above the 
theoretical ones, but the tendency is in good agreement between theory and experiment. The data obtained by the PAW-SQS method show good agreement with the experimental data, and also reproduce nonlinearity, as is the case with the experimental data. In addition, it should be noted that the results obtained by the PAW method predict a sharp change in Young's modulus.

It should be noted that upon the approach to the area of mechanical instability the results obtained by the PAW-SQS method predict a sharp decrease of the Young's modulus. This fact can be important to direct design of new alloys that are required by different applications.

\section{Ti-Ni-Al}

We have calculated the elastic properties of alloys in Ni-Al-Ti system. Calculations have been carried out by two first-principles theoretical methods, the EMTO-CPA and PAW-VASP.

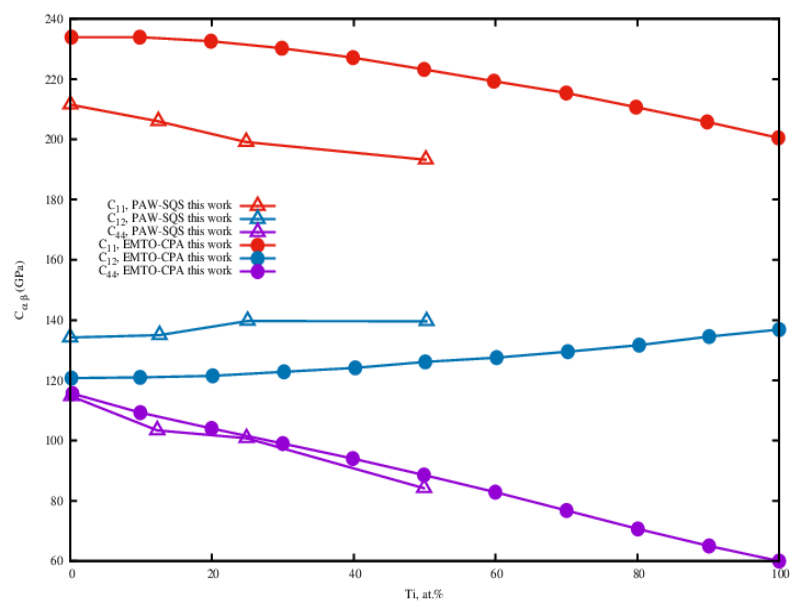

Fig.3.9. Dependence of elastic constants $\mathrm{C}_{11}, \mathrm{C}_{12}, \mathrm{C}_{44}$ on Ti concentration in the system $\mathrm{Ni}\left(\mathrm{Al}_{1-\mathrm{x}} \mathrm{Ti}_{\mathrm{x}}\right)$. These results suggest the possibility of proving the ductile properties of NiAl intermetallic by its alloying with Ti. The observed change in the elastic properties may indicate a change in the character of the chemical bonding of NiAl with increasing concentration of the alloying element Ti. It is known that $\mathrm{B} 2 \mathrm{NiAl}$ has the mixed type of interactions: the metallic bonding is predominant though covalent and ionic bonding are present as well. The decreasing value of $\mathrm{C}_{44}$ and improvement of the ductility characteristics with $\mathrm{Ti}$ addition indicates an increase in the degree of the metallic component in interatomic bonding. 

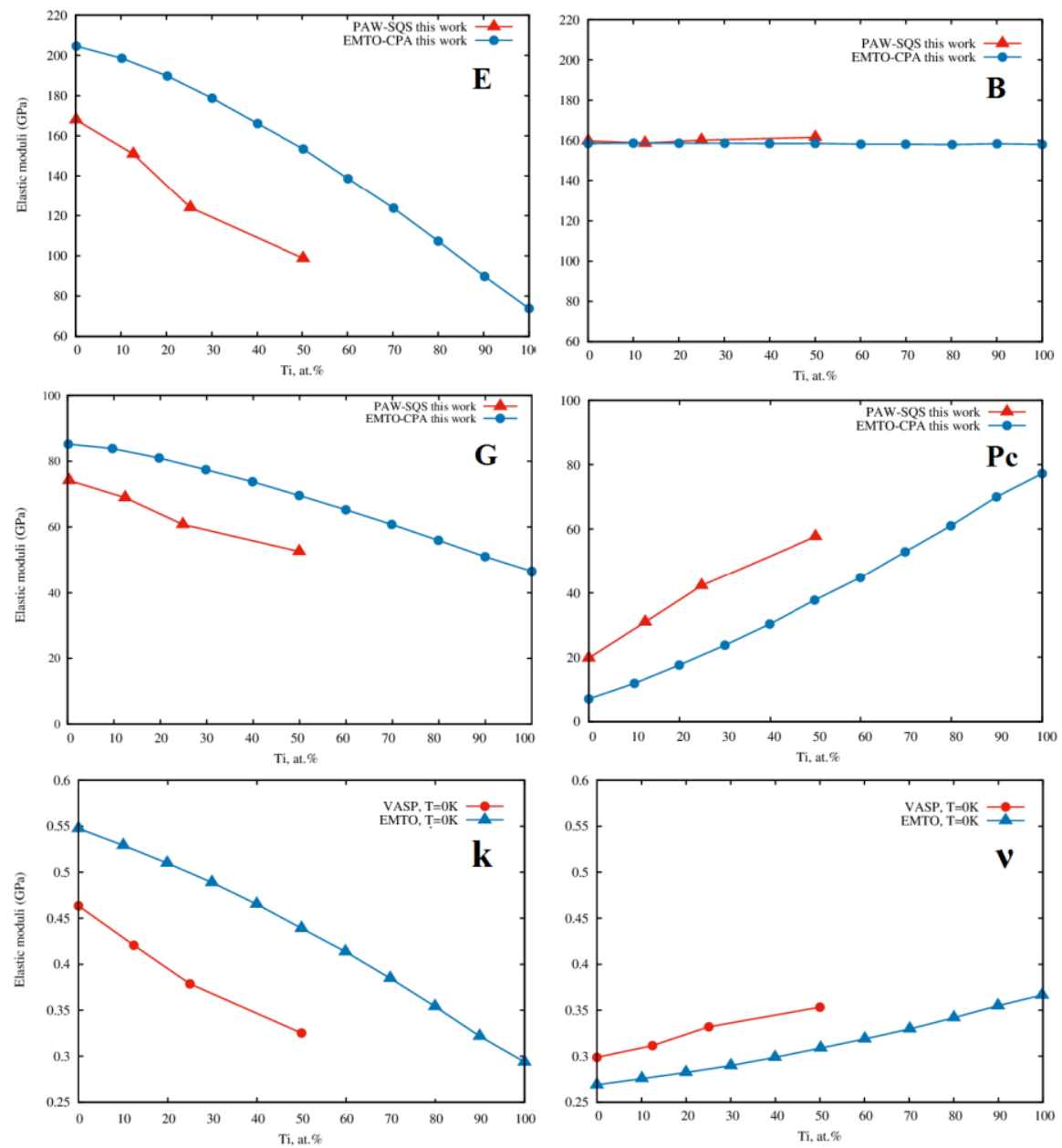

Fig. 3.10. Dependence of Young's modulus E, bulk modulus B, shear modulus $G$, Cauchy pressure $P_{c}$, the Pugh's coefficient $k$ and the Poisson coefficient $v$ on $\mathrm{T}$ concentration in the system $\mathrm{Ni}\left(\mathrm{Al}_{1-\mathrm{x}} \mathrm{Ti}_{\mathrm{x}}\right)$. The data obtained by the PAW-GGA method in this paper are denoted by red lines with red triangles for $\mathrm{E}, \mathrm{B}, \mathrm{G}, \mathrm{P}_{\mathrm{c}}$ and red circles for $k$ and $v$. The data obtained by the EMTO-CPA method are marked with blue lines, for E, B, G, $\mathrm{P}_{\mathrm{c}}$ blue filled circles and for the Pugh's coefficient $\mathrm{k}$ and Poisson coefficient $\mathrm{v}$ blue triangles.

Considering the effect of alloying NiAl with $\mathrm{Ti}$, our calculations show that it leads to a decrease of the material elastic modulus. For the uncertainty of the example, for $\mathrm{Ni}\left(\mathrm{Al}_{50} \mathrm{Ti}_{50}\right) \mathrm{E}$ is predicted to decrease to $182 \mathrm{GPa}$ in EMTO calculations and to $150 \mathrm{GPa}$ n PAW calculations. Most importantly, the variation of the Yong's modulus in $\mathrm{Ni}\left(\mathrm{AI}_{50} \mathrm{TI}_{50}\right)$ alloy in comparison to $\mathrm{NiAl}$ compound is in very good agreement between 
theoretical methods. One can quantitatively analyze the effect of alloying on other elastic moduli, such as elastic constants $\mathrm{C}_{11}, \mathrm{C}_{12}, \mathrm{C}_{44}$, bulk modulus B, Poisson's ratio v, shear modulus $\mathrm{G}$, $\mathrm{k}$ ratio and Cauchy pressure Pc (Fig. 3.9.-3.10). Moreover, the calculations reveal that $\mathrm{v}$ increases with increase of Ti concentration. It is worth noting that Pugh's coefficient $\mathrm{k}$ for the $\mathrm{Ni}(\mathrm{Al} 0.5 \mathrm{Ti} 0.5$ ) alloy is 1.5 times smaller and the value of the Cauchy pressure increased nearly four times compared with these valued for $\mathrm{B} 2 \mathrm{NiAl}$. These results suggest the possibility of improving the ductile properties of $\mathrm{NiAl}$ intermetallic by its alloying with Ti. The observed change in the elastic properties may indicate a change in the character of the chemical bonding of NiAl with increasing concentration of the alloying element Ti. It is known that $\mathrm{B} 2 \mathrm{NiAl}$ has the mixed type of interactions: the metallic bonding is predominant though covalent and ionic bonding are present as well. The decreasing value of $\mathrm{C}_{44}$ and improvement of the ductility characteristics with $\mathrm{Ti}$ addition indicates an increase in the degree of the metallic component in interatomic bonding.

Note that while EMTO calculations predict better values of the Young modulus for the alloy in comparison to our experiment, PAW appears to be somewhat more reliable in predicting the elastic modulus concentration variation. Moreover, within the accuracy offered by the phenomenological criterion of brittle vs ductile behavior, we can qualitatively analyze the effect of alloying on the ductility characteristics.

\section{Pure Ti}

In the present work, in the framework of the density functional theory, the high-temperature bcc-Ti phase was studied in the temperature range o-1700 K using AIMD. To determine the equilibrium lattice parameter at each temperature, MD was performed on a grid of volumes and temperatures, while we neglected the lattice entropy contribution to the pressure in the system [56]. Further, at each temperature, we used five distortion values $\delta=[-0.02,-0.01,0,0.01,0.02]$ and determined all the elastic constants of the cubic crystal from the corresponding stress-strain relations, as described above (see Fig. 3.11).

The calculations were performed for the $4 \times 4 \times 4$ cubic supercells containing 128 atoms. The special k-points mesh was set to $2 \times 2 \times 2$. The dynamic and static calculations were done using Methfessel-Paxton Brillouin-zone integration scheme with the smearing parameter equal to $0.20 \mathrm{eV}$. Exchange-correlation effects were treated using the generalized gradient approximation (GGA) in PBE parametrization [18]. Simulations were done within the projector augmented-wave (PAW) method. Plane wave cutoff energy was set to $460 \mathrm{eV}$. Nose-Hoover thermostat was applied in the AIMD simulations to obtain the canonical ensemble 
behavior. In the AIMD simulations the timestep was equal to $1 \mathrm{fs}$. The equilibration of the system usually took about $1 \mathrm{ps}$, and the averaging of the stress components was performed for the consequent time of $\sim 10 \mathrm{ps}$ for all temperatures except of $600 \mathrm{~K}$ where simulation time was $\sim 16 \mathrm{ps.}$

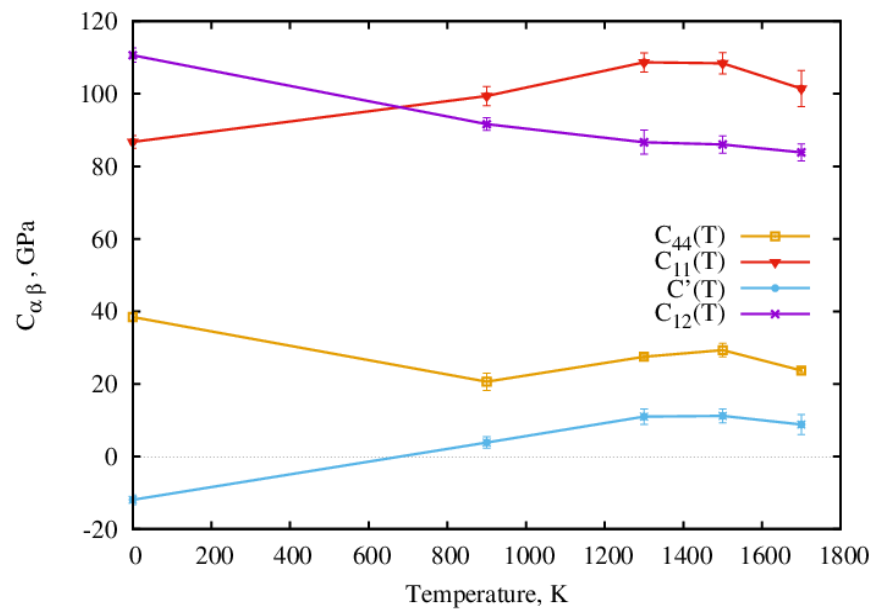

Fig. 3.11. Dependence of elastic constants $\mathrm{C}_{11}, \mathrm{C}_{12}, \mathrm{C}_{44}, \mathrm{C}^{\prime}$ on temperature in pure bcc Ti. Red line with triangular symbols corresponds to the calculated values of $\mathrm{C}_{11}$. Purple line with stars is the calculated values of $\mathrm{C}_{12}$. The yellow line with empty square symbols denotes the values of $\mathrm{C}_{44}$. Solid blue lines with filled circles are the values of C'.

\section{Elastic anisotropy}

The connection between compliances $\mathrm{s}_{\mathrm{ij}}$ and Young's modulus E can be obtained for a monocrystal. For cubic crystals Young's modulus for the direction with directional cosines $\alpha, \beta$, and $\gamma$ relative to crystallographic axes is given by

where

$$
\mathrm{E}=\left(\mathrm{s}_{11}-2 \mathrm{~s} \Gamma\right)
$$

$$
\begin{gathered}
\mathrm{S}=\mathrm{s}_{11}-\mathrm{s}_{12}+\frac{1}{2} \mathrm{~s}_{44}, \\
\Gamma=\alpha_{2} \beta_{2}+\alpha_{2} \gamma_{2}+\beta_{2} \gamma_{2}
\end{gathered}
$$

Accordingly, the directional dependence of the Young's modulus [38] for cubic crystals is calculated as

$$
\frac{1}{E_{\alpha \beta \gamma}}=\mathrm{S}_{11}-2\left(\mathrm{~S}_{11}-\mathrm{S}_{12}+\frac{1}{2} \mathrm{~S}_{44}\right)\left(\alpha_{2} \beta_{2}+\alpha_{2} \gamma_{2}+\beta_{2} \gamma_{2}\right)
$$


where $s_{\mathrm{ij}}$ is the average elastic compliances calculated from the averaged elastic constants $\mathrm{C}_{\mathrm{ij}}$.

Having all the elastic constants calculated, we have estimated Young's modulus and its directional dependence. Young's modulus E generally decreases with decreasing vanadium concentration. Interesting, the anisotropy of Young's modulus E changes in a peculiar way. In Fig. 3.12 the dependences of Young's modulus on the orientation of the crystal are given for different $\mathrm{V}$ concentrations.

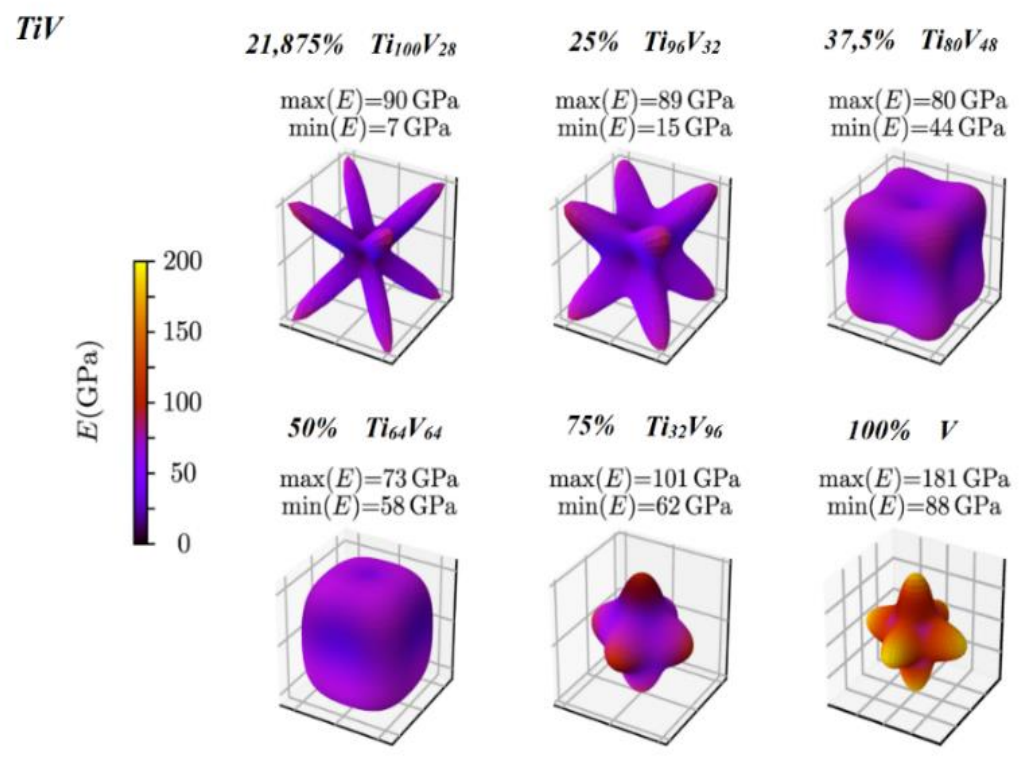

Fig. 3.12. Young's modulus as a function of direction for $\mathrm{V}$ concentrations in the bec Ti-V alloy from o to 100 at. \%.

The results are presented from o to 100 at. \% of vanadium in the Ti-V alloy for clarity of anisotropy. The minimum and maximum values shown in Fig. 3.11 were obtained on the uniform $(100 \times 50)$ azimuthalpolar grid of the unit sphere. The results reveal strong dependence of Young's modulus on both the direction and the $\mathrm{V}$ concentration.

As one can see from Fig. 3.12, in the range of $\mathrm{V}$ concentrations from 21.875 to 37.5 at. \%, close to mechanical instability, the directional dependence of Young's modulus is very pronounced. At V concentrations near 50 at. \% this dependence is rather weak. The obtained result indicates that a scheme of construction of monocrystals of Ti-V with the required value of Young's modulus can be based on the choice of the 
Chapter 3

orientation of the monocrystal. Materials with low Young's modulus, which correspond to those of polymer composite materials and biological tissues, are of high interest for applications. Compatibility of elastic moduli of monocrystals of Ti-V with elastic moduli of such materials will provide compatibility of deformations under external loads. 


\section{Mechanical stability}

The mechanical stability of the crystal lattice is its stability with respect to small distortions. It is determined from the generalized criterion for Born stability: at a given temperature (entropy), all determinants $\mathrm{D}_{\mathrm{k}}$ of the principal minors of the matrix $\mathrm{C}_{\mathrm{ij}}(\mathrm{i}, \mathrm{j}=1, \ldots, \mathrm{k})$ of isothermal (adiabatic) elastic moduli of the crystal $\mathrm{C}_{\mathrm{ij}}(\mathrm{i}, \mathrm{j}=1, \ldots, 6)$ in the Vogt notation have to be positive.

$$
\begin{gathered}
\mathrm{C}_{11}+2 \mathrm{C}_{12}>0 \\
\mathrm{C}_{11}-\mathrm{C}_{12}>0 \\
C_{44}>0
\end{gathered}
$$

So the mechanical stability can be determined by the criterion:

$$
\mathrm{C}^{\prime}>0
$$

where $\mathrm{C}^{\prime}$ is defined as:

$$
\mathrm{C}^{\prime}=\frac{1}{2}\left(\mathrm{C}_{11}-\mathrm{C}_{12}\right)
$$

Notice that mechanical stability does not necessarily mean thermodynamic stability, as there could be other phases that are lower in free energy.

\section{Details of calculations}

Calculation details are given in chapter 3 .

\section{Results}

\section{Ti-Ni-Al}

We confirmed that for Ni(Al1-xTix) alloys the criteria for a mechanically stable cubic crystal, i.e. Eqs. (4.3) and (4.4), are satisfied in the whole concentration interval. 
Fig 4.1 illustrates the behavior of $\mathrm{C}_{44}$ as a function of Ti concentration. It is nicely described by a linear function.

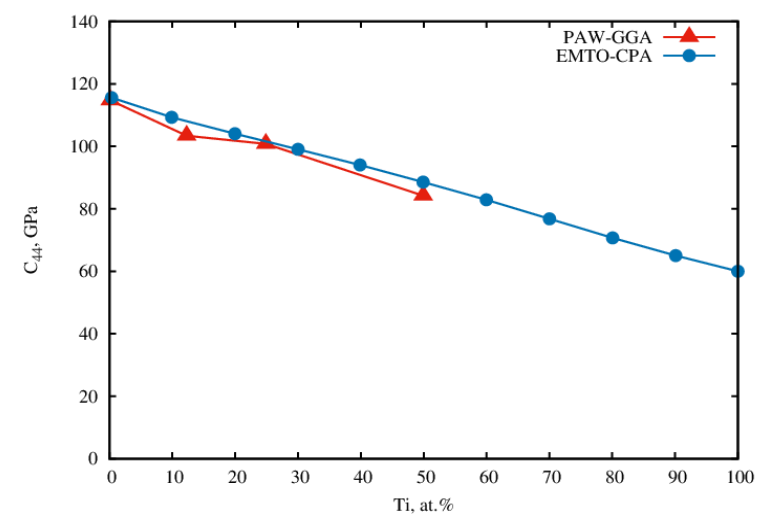

Fig. 4.1. Dependence of the elastic constant $\mathrm{C}_{44}$ on Ti concentration in the system $\mathrm{Ni}\left(\mathrm{Al}_{1-\mathrm{x}} \mathrm{Ti}_{\mathrm{x}}\right)$.

\section{Ti-V}

To determine the mechanical stability of the bcc Ti-V alloys, we note that bulk modulus and $\mathrm{C}_{44}$ are always positive, as seen in Figure 4.2.

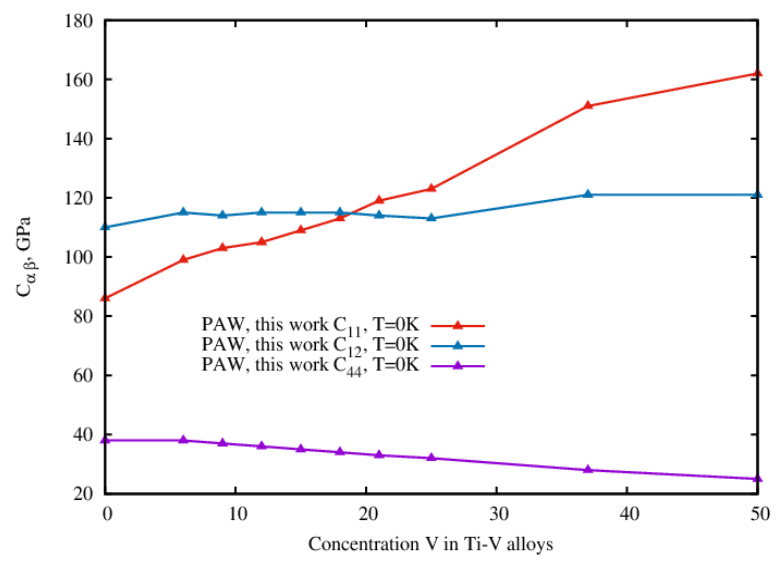

Fig. 4.2. Dependence of the elastic constant $C_{\alpha \beta}$ on $V$ concentration in bcc Ti-V. Red symbols correspond to the calculated values of $\mathrm{C}_{11}$. The calculated values of $\mathrm{C}_{12}$ are shown as the blue symbols. Purple symbols are the calculated values of $\mathrm{C}_{44}$. 
Fig. 4.3. shows the calculated dependence of the elastic constant $C^{\prime}$ on the $\mathrm{V}$ concentration in the Ti-V alloy, obtained by the PAW-SQS method.

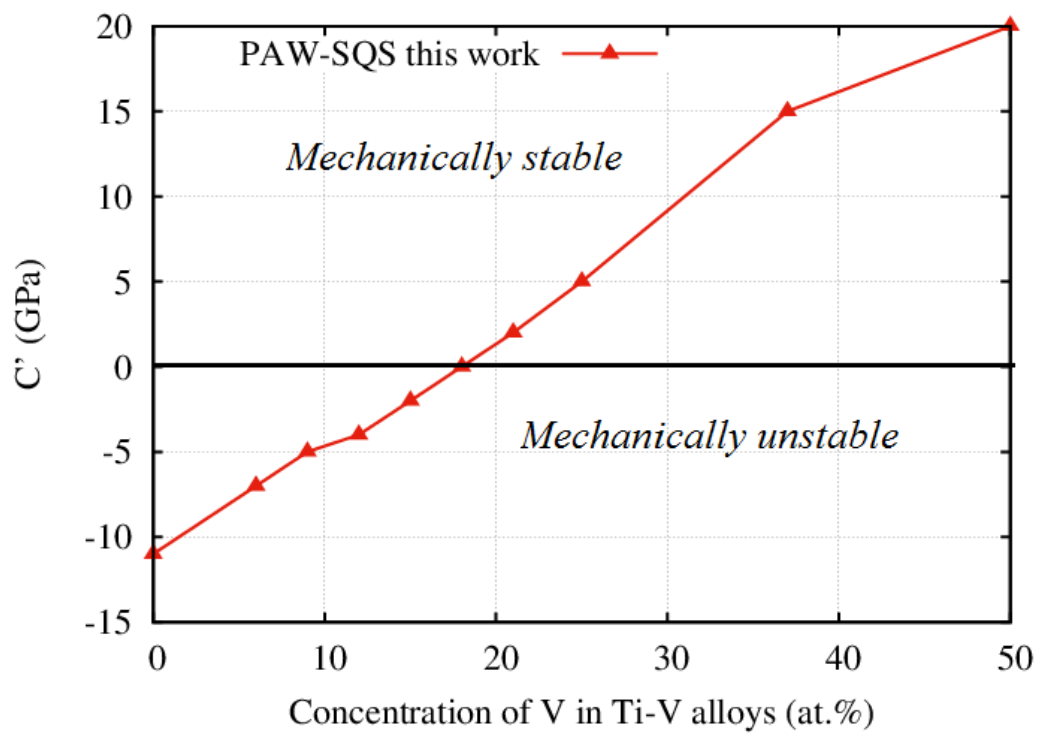

Fig. 4.3. Dependence of the elastic constant $\mathrm{C}^{\prime}$ on $\mathrm{V}$ concentration in bcc Ti-V.

The elastic constant $\mathrm{C}^{\prime}$ obtained by the PAW method monotonically increases with increasing vanadium concentration. The dependence $\mathrm{C}^{\prime}\left(\mathrm{X}_{\mathrm{V}}\right)$ is weakly nonlinear. The elastic constant $\mathrm{C}^{\prime}$ increases with the rise in content of the $\mathrm{V}$ doping element. As follows from Figure, the mechanical stability of bcc Ti-V alloys increases with $\mathrm{V}$ content. In particular, the bcc structure is mechanically stable when the content of $\mathrm{V}$ is more than 18 at. \% according to the requirements of the mechanical stability for a cubic crystal.

It was found that rich titanium alloys are mechanically unstable, but the mechanical stability increases with increasing percent content of $\mathrm{V}$ in the system. 


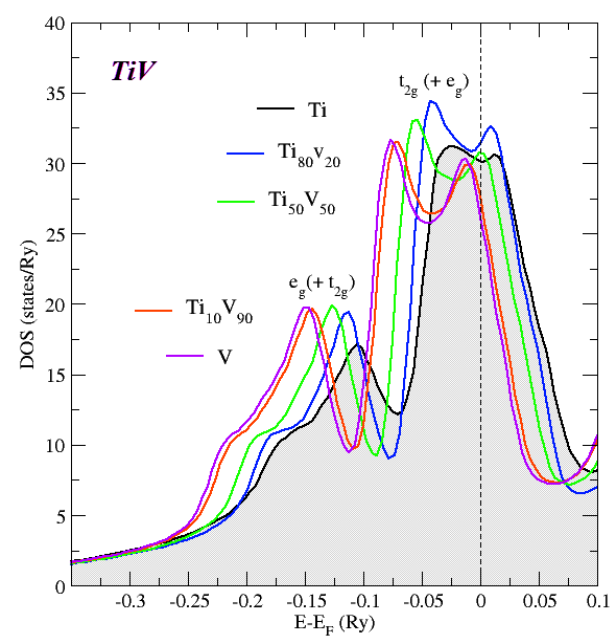

Fig.4.4 - Calculated total density of states of Ti-V alloys.

The analysis of the density of electronic states (DOS) (Figure 4.4 ) shows that strengthening of metallic bonding is provided, mainly, by the increasing occupation of $\mathrm{t}_{2 \mathrm{~g}}$ and $\mathrm{e}_{\mathrm{g}}$ orbitals by d-electrons, which leads to a decrease of directional electron charge density distribution that becomes more spherical. It is seen from Fig. 4.4 that for the considered alloys consisting of Ti and $\mathrm{V}$ - neighboring elements in the periodic table - the rigid-band approximation works well. In fact, when vanadium is added, the number of valence electrons increases, and the Fermi level shifts toward the higher energy practically without changing the shape of the band. The main deviation from the approximation is an increase in the peak height located in the interval -0.2 - -0.1 Ry and formed predominantly by the states of $e_{g}$ symmetry. Since $e_{g}$-orbitals in bcc-type structures provide an atomic bond between the second neighbors, this effect leads to an increase in the elastic constant $\mathrm{C}_{11}$ and, as a result, to the mechanical stabilization of the alloy. It should be noted, that the applicability of the rigid-band model is reflected in the good agreement of our PAW-SQS results and the data obtained by using the virtual crystal approximation (VCA) in Ref. [53] for the elastic constant C' (Fig. 4.3. ).

\section{Pure Ti}

Bcc-phase of pure $\mathrm{Ti}$ is an unstable system at low temperatures.

$A b$ initio molecular dynamics (AIMD) simulations have been applied to calculate the temperature dependent elastic constants of pure bcc Ti. 


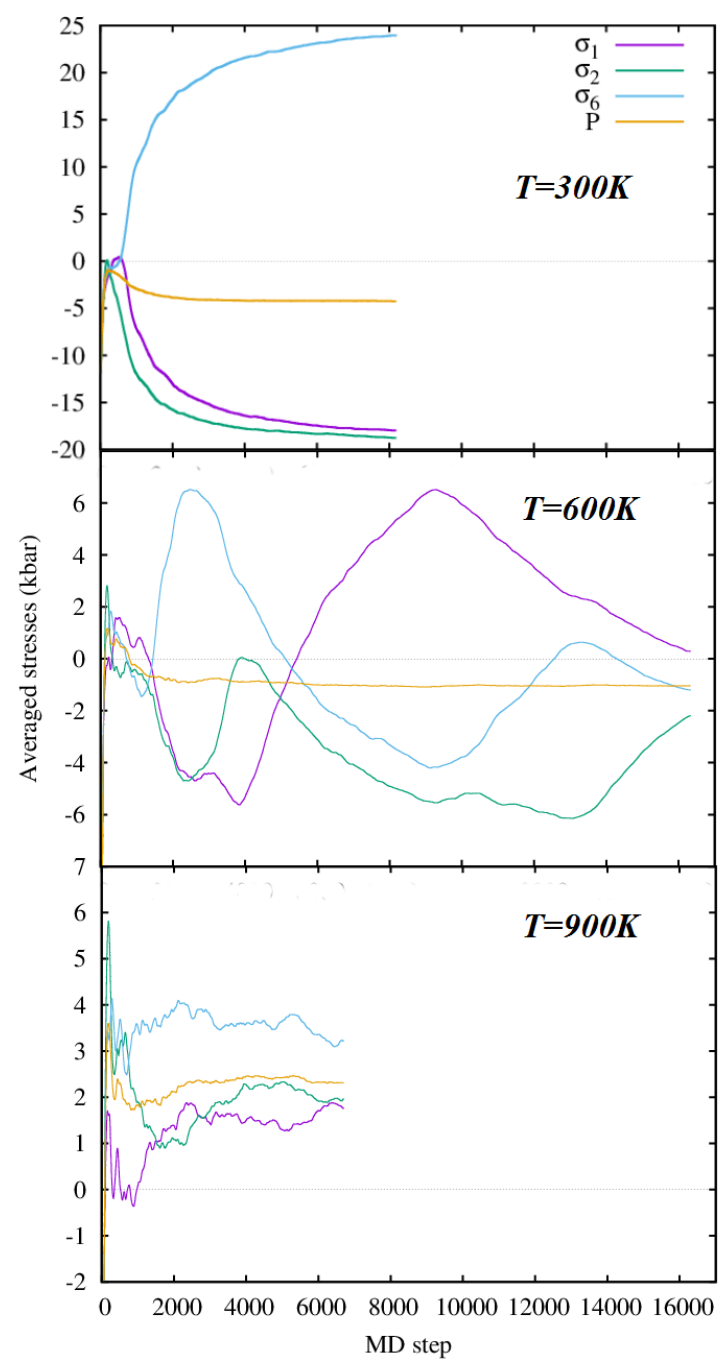

Fig. 4.6. Dependence stress values $\left(\sigma_{i}\right)$ and the pressure averaged over the MD steps on number of MD-steps at various temperatures: a) $300 \mathrm{~K}$, b) $600 \mathrm{~K}$ and c) $900 \mathrm{~K}$. stress $\sigma_{\mathrm{XX}} \sigma_{\mathrm{YY}} \sigma_{\mathrm{ZZ}}$.

The system is unstable at $\mathrm{T}=300 \mathrm{~K}$. Average stresses demonstrate large oscillations at $\mathrm{T}=600 \mathrm{~K}$. At $\mathrm{T}=900 \mathrm{~K}$ the system is stable, as can be seen after relatively small number of time steps.

Let us consider in more detail the case with system at $\mathrm{T}=600 \mathrm{~K}$. In this case 5 different deformations have been applied and strains above $2 \%$ lead to disappearance of the oscillations of stresses. Therefore, the system becomes mechanically unstable. One can see this in Fig. 4.7. 

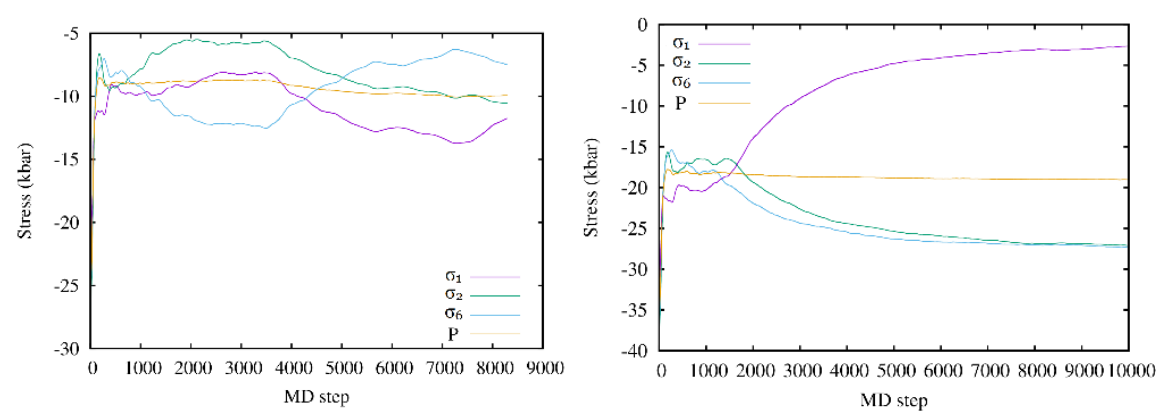

Fig. 4.7. Dependence stress values $\left(\sigma_{\mathrm{i}}\right)$ and the pressure averaged over the MD steps on number of MD-steps at $600 \mathrm{~K}$ for different deformations.

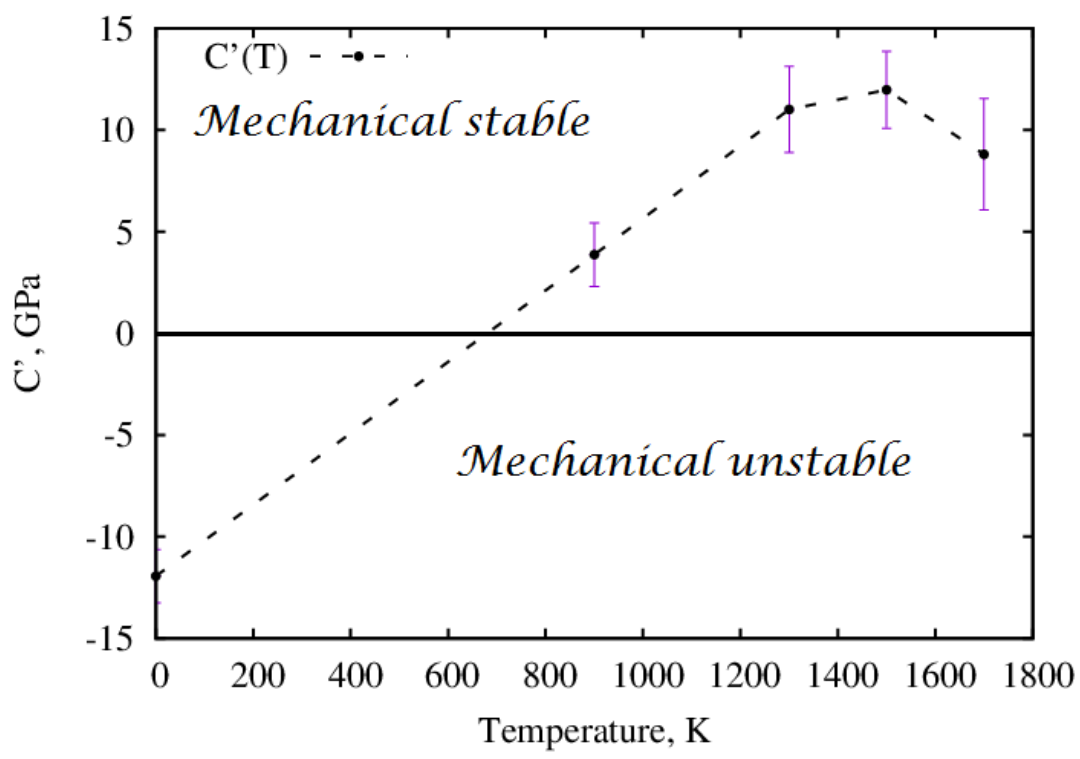

Fig. 4.8. Dependence of the elastic constant $\mathrm{C}^{\prime}$ on temperature for pure Ti. The stabilization of $\mathrm{Ti}$ with increasing temperature is noticeable. Bcc structure of $\mathrm{Ti}$ is stable when the temperature is above $700 \mathrm{~K}$ according to the requirements of the mechanical stability for a cubic crystal. The system is mechanically stable at $\mathrm{T}=1300 \mathrm{~K}$ and $\mathrm{T}=900 \mathrm{~K}$. $\mathrm{C}^{\prime}>0$

We reproduce the mechanical stabilization of pure bcc Ti under heating. Bcc structure of $\mathrm{Ti}$ is stable when the temperature is above 700 $\mathrm{K}$ according to the requirements of the mechanical stability for a cubic crystal. The mechanical stability at $700 \mathrm{~K}$ is not sufficient to stabilize bcc phase with respect to hcp phase. The thermodynamic stability of bcc phase achieved at higher temperature, $\mathrm{T}=1155 \mathrm{~K}$. 


\section{Ti-V at high temperature}

The average atomic positions indicate that the atoms are close to the ideal bcc at $1300 \mathrm{~K}$, though at zero $\mathrm{K}$ the atoms move out of the ideal bcc positions creating the unphysical non-bcc structure within the $4 \times 4 \times 4$ supercell, as expected in the dynamically unstable system. Certainly, if the shape of the supercell would not be conserved during the simulation, it could be further destroyed, and the structure could change to, for example, hcp.
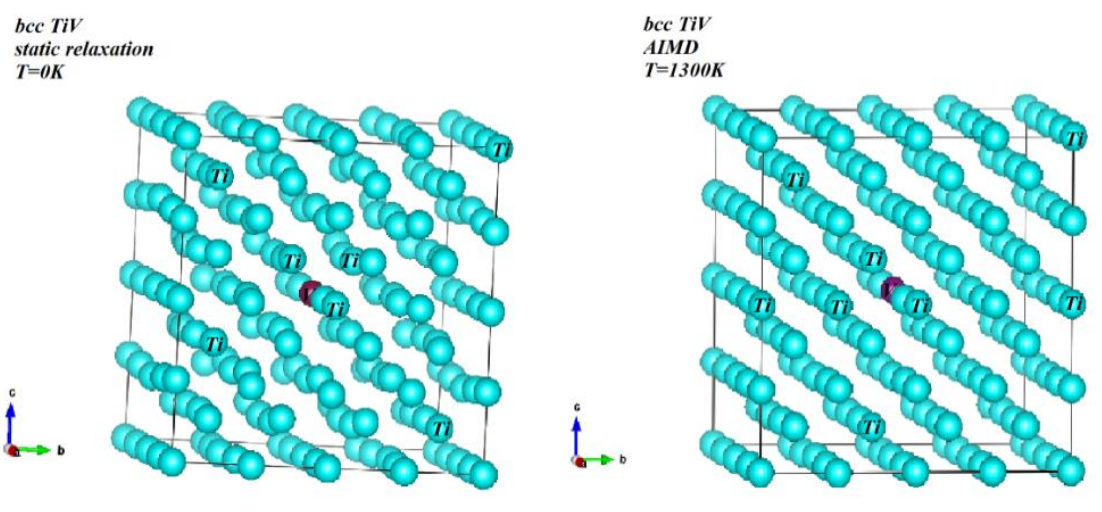

Fig. 4.5. The bcc crystal lattices of TiV alloy obtained by static relaxation of the supercell at $\mathrm{OK}$ and from AIMD at $1300 \mathrm{~K}$ as the time-average of the atomic positions. 

Chapter 5

\section{Mixing Enthalpy}

Mixing enthalpy is the energy released or absorbed by a substance when mixed. The enthalpy of mixing is the result of new interactions between substances that combine with each other. In ideal mixtures, the enthalpy of mixing is zero. In non-ideal mixtures, the thermodynamic activity of each component differs from its concentration by multiplication by the activity coefficient.

\section{Details of calculations}

Using the EMTO-CPA and PAW-SQS methods, the thermodynamically properties of bcc $\mathrm{Ti}-\mathrm{V}$ alloys in the whole concentration range were calculated. Experimental measurements of the mixing enthalpy of Ti-V alloys in the concentration range from 10 to 20 at. \% of $V$ were also carried out. In the SQS calculations on the 128 atom cell of $\mathrm{Ti}-\mathrm{V}$, formation enthalpy $\Delta \mathrm{H}$ was determined as the difference in energy of the Ti-V system and the energies of pure Ti and V systems:

$$
\Delta \mathrm{H}=\mathrm{E}_{\mathrm{Ti}(128-\mathrm{n}) \mathrm{V}}-\left((128-\mathrm{n}) / 128 \mathrm{E}_{\mathrm{Ti}}+\mathrm{n} / 128 \mathrm{E}_{\mathrm{V}}\right)
$$

where $E_{T i}$ and $E_{V}$ are the energies of the pure Ti and V, respectively, $\mathrm{n}$ is the number of vanadium atoms in the supercell, $E_{T i(128-n) V}$ is the energy of the alloy with $n$ vanadium atoms in the SQS. All the energies were calculated at the respective equilibrium volumes.

\section{Results}

Figure 6.2 shows the calculated formation enthalpies for the range from $\mathrm{o}$ to 100 at. \% of vanadium in the Ti-V alloy. 


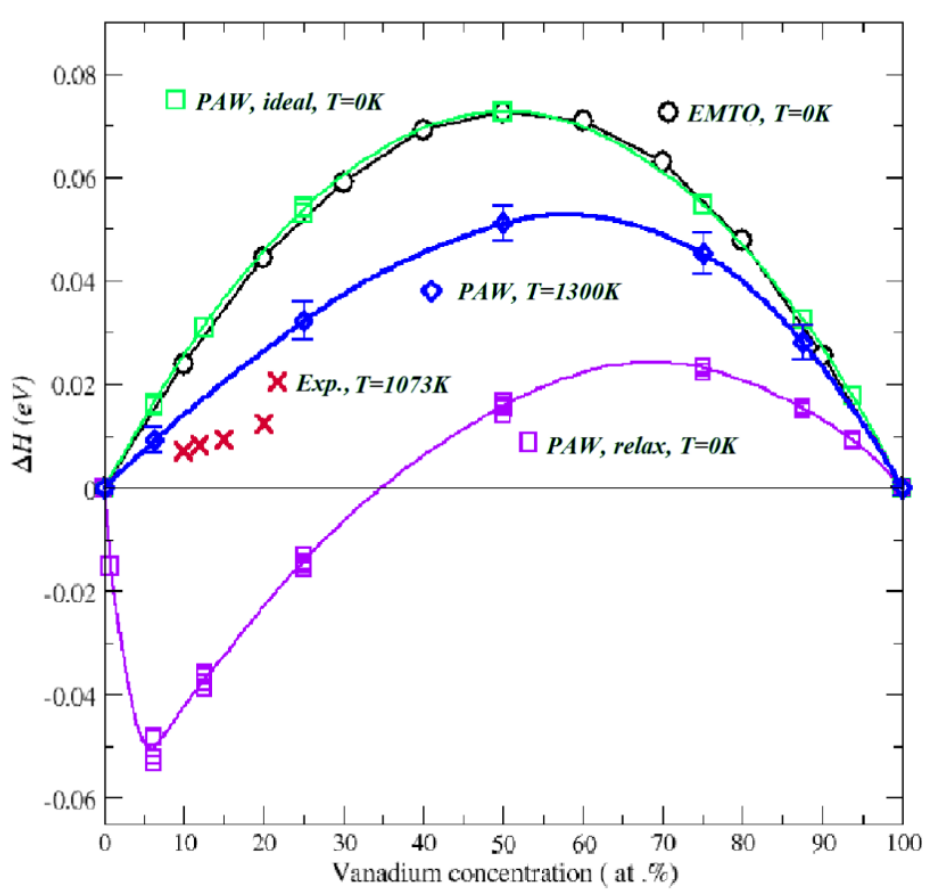

Fig. 6.2. Dependence of mixing enthalpy on $\mathrm{V}$ concentration in bcc Ti-V alloy. Black symbols correspond the obtained values by the EMTO-CPA method in this work. The empty square symbols denote the obtained values by the PAWSQS method at the $\mathrm{T}=\mathrm{o} \mathrm{K}$. Green symbols are the values obtained for the ideal bcc structure, purple symbols are the values obtained for the relaxed structure.

The values obtained in this paper with the PAW method at $\mathrm{T}=1300 \mathrm{~K}$ are shown as the blue symbols. Red symbols are experimental values.

The enthalpies calculated by PAW-SQS with ideal bcc positions and by EMTO-CPA at o K agree with each other and show positive values and the qualitatively correct upward curvature in the whole concentration range. However, they lie substantially above the experimental points, i.e. the curvature of the calculated at o K enthalpy is overestimated.

AIMD simulations was applied to calculate the mixing enthalpy at $1300 \mathrm{~K}$. The new curve of the formation enthalpy at $1300 \mathrm{~K}$ is in better agreement with the available experimental points. In this case of a dynamically unstable at o $\mathrm{K}$ structure it is not enough to calculate the enthalpy for the average structure, i.e. bcc in case of Ti-V. It is necessary to take into account how the atoms vibrate and take the average of the enthalpy over the structures arising in MD. 
Relaxation of the bcc structure (which is dynamically unstable at o K) while keeping the cubic shape of the supercell, leads to the formation of

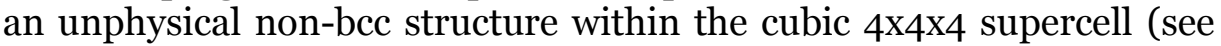
Fig. 6.2). Therefore, although the formation enthalpy of this structure is negative for vanadium concentrations below 35 at. \%, the bec structure is not stabilized.

Figure 6.3 shows the dependence of the mixing enthalpy on $\mathrm{V}$ concentration in bcc Ti-V alloy with respect to bcc $\mathrm{V}$ and hep Ti. If one considers the formation enthalpy with respect to the hcp rather than bcc $\mathrm{Ti}$, that it can be seen the hcp $\mathrm{Ti}$ is stable at $\mathrm{O} \mathrm{K}$ and the formation enthalpy of bcc Ti-V alloys in this case is positive.

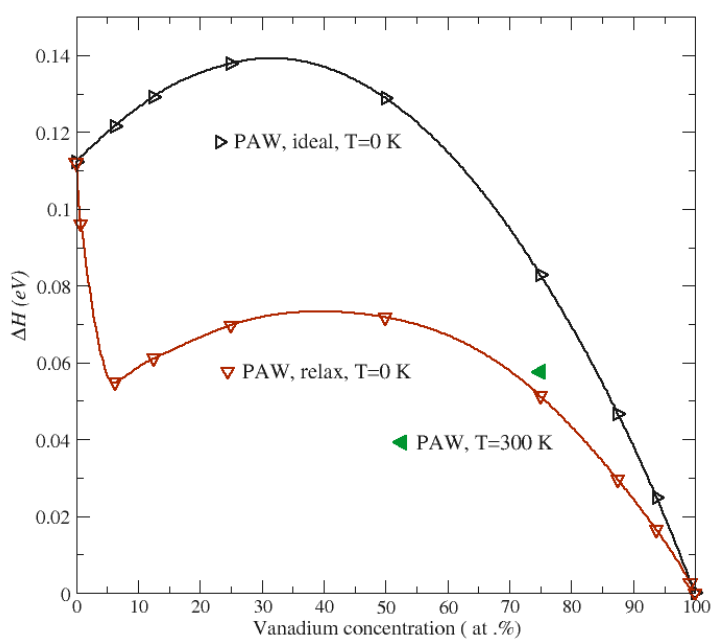

Fig. 6.3. Dependence of the mixing enthalpy on $\mathrm{V}$ concentration in bcc Ti-V alloy with respect to bcc $\mathrm{V}$ and hcp Ti. Black solid line with empty triangular symbols are the values obtained in this paper with the PAW-SQS method at $\mathrm{T}=\mathrm{o} \mathrm{K}$ for the ideal bcc structure. The obtained values by us with PAW method at $\mathrm{T}=\mathrm{O} \mathrm{K}$ for relaxed structure are indicated by the red empty triangular symbols. The values obtained by AIMD at $\mathrm{T}=300 \mathrm{~K}$ are designated by green filled triangular symbols.

To summarize, the formation enthalpies of bcc $\mathrm{Ti}-\mathrm{V}$ alloys were calculated from first principles at $\mathrm{O}$ and $1300 \mathrm{~K}$ as a function of the vanadium concentration. We found that enthalpies of bcc Ti-V alloys are positive in the whole concentration range of vanadium at $\mathrm{o} \mathrm{K}$, though calculations with static relaxation of atomic positions predict negative values at low vanadium concentration. It is shown to be because the bcc structure of Ti is dynamically unstable at o K, so the relaxed structure of the supercell is actually not bcc. If one considers its formation enthalpy with respect to hcp $\mathrm{Ti}$, it becomes positive at all $\mathrm{V}$ concentrations. 
Chapter 5

Calculations of the formation enthalpy at $1300 \mathrm{~K}$ give much better agreement with the experimental data and show that it is important to take into account vibrations of the atoms obtained in molecular dynamics simulations. 
Chapter 6

\section{Diffusion}

The knowledge of the laws of diffusion processes allows a specialist to significantly increase the efficiency of searching for new materials with desired properties and optimal methods for their processing. Diffusion plays a huge role in determining the structure and properties of materials.

Diffusion in a solid is a spontaneous process of the redistribution of atoms in space. Sometimes atoms can move at interparticle distances in the lattice. The driving force of diffusion is the reduced free energy of the crystal (chemical potential difference at different points in the system). $\mathrm{F}=\mathrm{U}-\mathrm{TS}$, where U is internal energy, and $\mathrm{S}$ is entropy. Diffusion leads to atomic mixing, that is, to a decrease in the degree of order in the system. When the lattice is perfect, diffusion is difficult, since a large deformation of the lattice should occur. It becomes possible when an atom is missing. In this case, the distortions created by the diffusing atom are significantly smaller. A measure of diffusion is the diffusion coefficient. The diffusion coefficient is a quantitative characteristic of the diffusion rate equal to the amount of matter passing through a portion of a unit area per unit time as a result of the thermal motion of particles with a concentration gradient.

The dependence on temperature of the diffusion coefficient for solids is determined by the Arrhenius equation:

$$
D=D_{0} \exp \left(-\frac{E_{a}}{R T}\right)
$$

where $\mathrm{D}$ is the diffusion coefficient, $\mathrm{D}_{\mathrm{o}}$ is the maximal diffusion coefficient at infinite temperature, $\mathrm{E}_{\mathrm{a}}$ is the activation energy for diffusion, $\mathrm{R}$ is the universal gas constant, and $\mathrm{T}$ is the absolute temperature.

Available experiments indicate a breakdown of the Arrhenius law for diffusion coefficient, D, in the case of bcc Ti. However, experimental D values depend on both migration rates and vacancy equilibrium concentrations. Therefore, the question is whether the overall nonArrhenius D behavior is due to a non-Arrhenius trend in both quantities or just one of them. Pure Ti has bcc-structure at high temperatures. The bcc phase of pure Ti is unstable at low temperatures up to $1152 \mathrm{~K}$ [57]. 


\section{Details of calculations}

AIMD simulations of defect-free bcc $\mathrm{Ti}(5 \times 5 \times 5)$ conventional bcc cells, (250 atoms) were carried out by sampling the canonical NVT configuration space at a temperature of $1800 \mathrm{~K}$, the Ti melting point $\mathrm{T}_{\mathrm{m}}$ $\approx 1940 \mathrm{~K}$. The Brillouin zone integrations were performed using a set of $2 \times 2 \times 2 \mathrm{k}$-points. The cut-off energy was chosen to be $300 \mathrm{eV}$ for bcc pure Ti.

AIMD simulations have been applied to calculate a diffusion coefficient $\mathrm{D}$ at different temperatures. The bcc structure of pure Ti were described with the 127-atom supercell constructed as a $4 \times 4 \times 4$ simple-cubic supercell without 1 atom. The calculations were carried out for such temperatures as $900 \mathrm{~K}, 1000 \mathrm{~K}, 1100 \mathrm{~K}, 1500 \mathrm{~K}, 1700 \mathrm{~K}$. We used 127 atom supercell. Mean squared displacement (MSD) is measured over time, which allows you to determine which parts are serious due to diffusion or the advective force also contributing. MSD is a measure of the deviation of the particle's position from the position at the initial time.

$$
\begin{gathered}
\mathrm{D}=\lim _{\mathrm{t} \rightarrow \infty} \frac{1}{6 t} \mathrm{MSD}(\mathrm{t}) \\
\mathrm{MSD}=\frac{1}{N} \sum_{n=1}^{N}\left(x_{n}(t)-x_{n}(0)\right)^{2},
\end{gathered}
$$

where $\mathrm{t}$ is time, $x_{n}(t)$ is the position of atom $\mathrm{n}$ at time $\mathrm{t}$, and the summation in Eq. (7.3) is done over all $\mathrm{N}$ atoms.

\section{Results}

\section{Non-Arrhenius behavior of diffusion coefficient in bce Ti}

This anomaly of non-Arrhenius behavior of diffusion coefficient in bcc Ti is observed when approaching the temperature corresponding to the unstable region.

Our investigations aim to determine on whether vacancy migration rates are strongly non-Arrhenius in the vicinity of instability. 


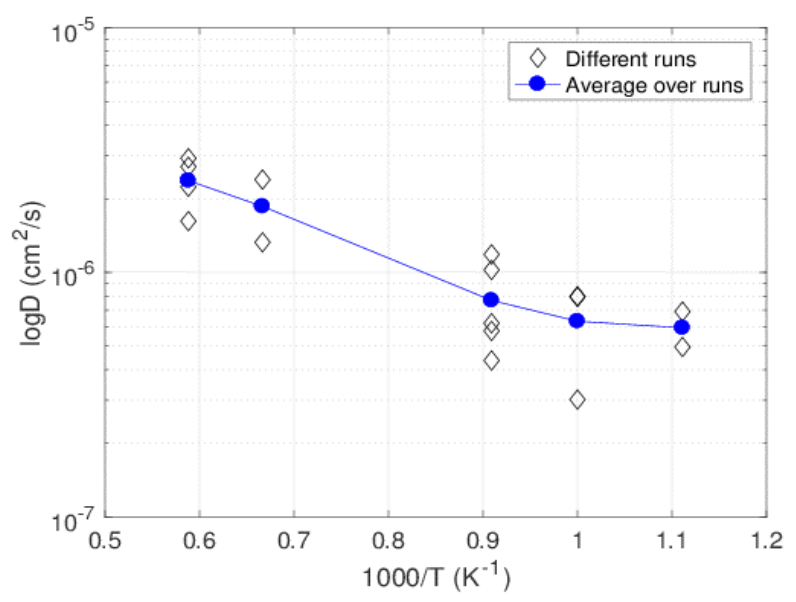

Fig. 7.1. Vacancy-concentration-normalized diffusion coefficients in bec $\mathrm{Ti}$ determined as a function of temperature.

Fig. 7.1 shows calculated vacancy-concentration-normalized diffusion coefficients in bcc $\mathrm{Ti}$ as a function of temperature. It is clear that $\mathrm{D}$ is strongly non-Arrhenius at lower temperatures, in good correspondence with the proximity to the thermodynamic instability at around $1152 \mathrm{~K}$ [57].

\section{Self-diffusion in bce Ti}

We investigated the diffusion properties of bcc Ti near the instability. Our studies were aimed at determining whether the non-Arrhenius vacancy migration rates are strong in the vicinity of the dynamic instability. We wanted to see how the transition from the unstable to stable bcc phase could affect its self-diffusion.

From temperatures of $\sim 80 \%$ of the $\mathrm{Ti}$ melting point, concerted migration in defect-free lattice regions becomes the dominant diffusion mechanism, that is, the calculated self-diffusion coefficient is essentially unaffected by the presence of point defects.

We obtained rate of a diffusion process at temperature $1800 \mathrm{~K}$.

We obtain "Rapid" spontaneous formation of Frenkel pairs in defectfree bcc Ti. It is indicative of very low defect formation energies in strongly anharmonic systems. With enough excess energy, an atom jumps into the interstitial space and does not return back but rather goes on in the interstitial space. 
Chapter 6

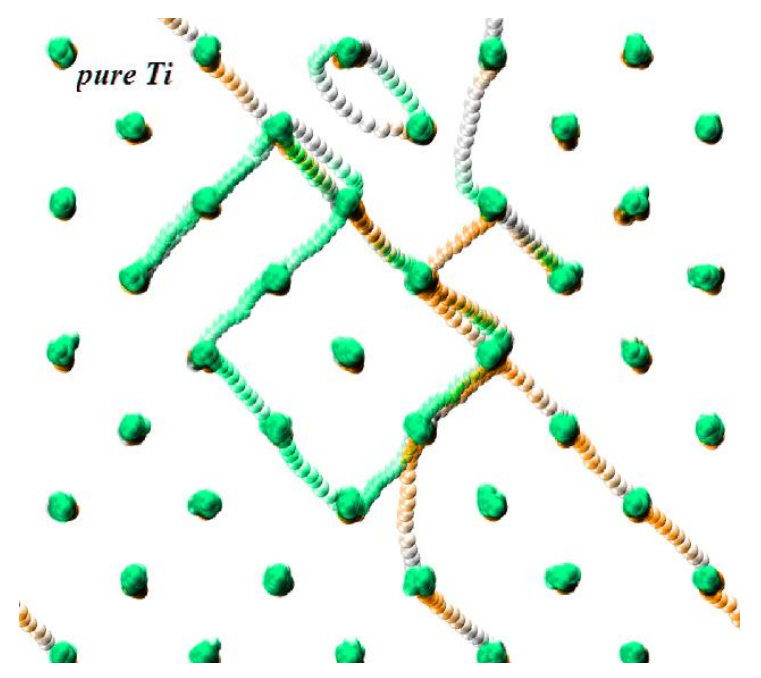

Fig. 7.2. Orthographic view on a bcc Ti(001) plane of superposed AIMD snapshots during concerted Ti migration at $1800 \mathrm{~K}$. The color variation, green $\rightarrow$ white $\rightarrow$ golden, is indicative of time progression during $\approx 9$ ps. For clarity of visualization, trajectory-smoothing over 1000 steps (1 ps) is used to reduce the vibrational noise caused by strong lattice anharmonicity.

More important, the spontaneous formation of Frenkel pairs (with no quick annihilation (recombination) of vacancy or interstitials) is done in a concerted manner, so collective motion of Ti atoms is observed. This is the reaction pathway for formation of defects in a defect free lattice. The pathway itself is of interest: thermodynamics tells that any lattice contains defects but does not tell how these defects are actually formed. Therefore, we revealed an interesting mechanism for high-temperature diffusion in bec titanium. 


\section{Chapter 7}

\section{High Entropy Alloys}

High-entropy alloys (HEA)[58] are the systems containing 5 or more elements, usually with equal or nearly equal concentrations, that are obtained as disordered alloys, typically on close-packed or nearly closepacked structures, like the body-centred cubic (bcc) one. They can be obtained by different techniques, including traditional casting, mechanical alloying, sputtering, splat-quenching, etc. There are many HEAs with different alloying elements that have been produced and whose microstructures and mechanical, thermal, and electronic performances have been studied experimentally.

Though HEAs were discovered more than 30 years ago, an active research has been done just for the past 10 years. The reason is that some attractive properties of HEAs have been reported. In particular, there are reports that HEAs have better fracture resistance, tensile strength, as well as corrosion and oxidation resistance, than conventional alloys. In particular, these alloys are considered for the possible storage of hydrogen or as systems that can contain excessive amount of carbon. The calculations of such alloys are often done with methods that can treat disorder of chemical elements on the crystalline lattice but cannot relax the atomic positions. However, if the hydrogen storage or carbonization are addressed, it is important to know the sizes of the intrinsic structural voids, where hydrogen or carbon can go. Obviously, in case of atomic relaxations the shape and size of these voids may change. Though relaxations were already considered for some HEAs [59], their effect on the voids was not addressed. Our aim was to investigate this question in a typical bcc-based HEA, the equiatomic Ti-Hf-Nb-V-Zr alloy.

\section{Method}

For the modeling of the Ti2O-Hf2O-Nb2O-V2O-Zr2O alloy, within density functional theory (DFT) a supercell has been set up that is of reasonable size to account for relaxations and which allows for correct equal concentrations, while being small enough to run relatively quick

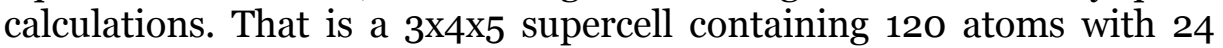
atoms of each element. As the atomic distribution in actual HEA is not known and can be away from thermodynamic equilibrium depending on the preparation process and kinetics, the calculations were carried out for 2 atomic distributions modeled using the special quasirandom structures (SQS) technique. One was set up for a completely random alloy (see Fig. 8.1), i.e. all 10 independent pair short-range ordered parameters were closely matching those of the perfectly random alloy up 
at least 3 coordination shells; and the second for maximally clustered elements inside the supercell (see Fig. 8.2), i.e. the case of elemental phase separation. Our calculations were done with Vienna Ab Initio Simulation Package (VASP) [22], [43], [44] within projector-augmented wave (PAW)[21] method using generalized gradient approximation (GGA)[18] for exchange and correlation. The value of the cutoff energy for plane waves was set to $460 \mathrm{eV}$. The Monkhorst-Pack k-point grid for the Brillouin zone (BZ) integration contained 4 points in the irreducible wedge of BZ. The Gaussian smearing with the smearing width of $0.2 \mathrm{eV}$ was applied. The relaxation of the internal degrees of freedom for calculated equilibrium volumes until the forces on all the atoms were less than $0.01 \mathrm{eV} / \AA$. For the calculations of void volumes the cubic shape was kept. We notice that tests with full relaxation of volume, shape, and atomic positions were also done. The observed deviations from the cubic shape were negligibly small, indicating that the chosen SQSs effectively conserve cubic shape to a large degree.

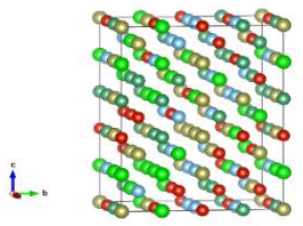

ideal bcc positions

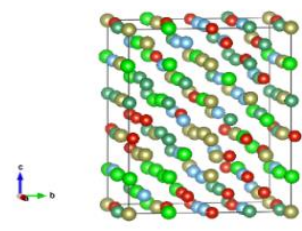

relaxed positions
Hf-Nb-Ti-V-Zr

SQS

$3 \times 4 \times 5$

120 atom supercell

24-24-24-24-24

Fig. 8.1. D model of Hf-Nb-Ti-V-Zr alloy modelled as a 120 atom random alloylike supercell with ideal bcc structure and the same structure after static atomic relaxations.

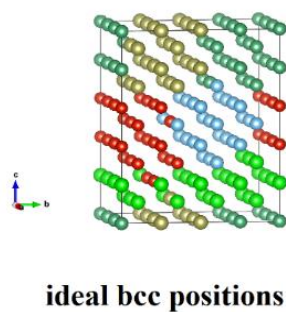

ideal bec positions

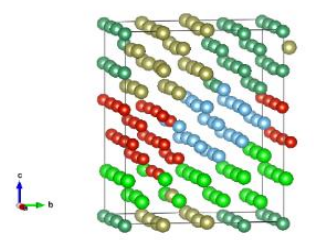

relaxed positions
Hf-Nb-Ti-V-Zr

Separation

$3 \times 4 \times 5$

120 atom cell

24-24-24-24-24

Fig. 8.2. D model of Hf-Nb-Ti-V-Zr alloy modelled as a 120 atom phase elements separated-like supercell with ideal bcc structure and the structure after static relaxations. 


\section{Results and discussion}

The relaxations of atomic positions turned out to be really important. Though atoms do not move by large distances, the effect on the total energy is big.

For unrelaxed structures the random atomic distribution is lower in energy than the elemental phase separated case,namely

Esep - Ernd $=47.3 \mathrm{meV} /$ atom,

where Esep is the energy for the structure with clustered elements and $\mathrm{E}_{\text {rnd }}$ is the energy for the structure with random distribution of elements

However, when the atomic positions are relaxed, the situation is the opposite, namely

Esep - Ernd $=-20.4 \mathrm{meV} /$ atom

For the phase separated structure the difference of energy of the structure without relaxations and the energy of structure after relaxation is:

$\mathrm{E}_{\text {unrelax }}-\mathrm{E}_{\text {relax }}=187.4 \mathrm{meV} /$ atom

For the SQS structure with the random distribution of atoms the difference between those energies is:

Eunrelax $-E_{\text {relax }}=119.6 \mathrm{meV} /$ atom, i.e. smaller by almost 70 $\mathrm{meV} /$ atom.

The formation enthalpy was calculated as:

$$
\Delta \mathrm{H}=\mathrm{E}(\mathrm{Hf}-\mathrm{Nb}-\mathrm{Ti}-\mathrm{V}-\mathrm{Zr})-0.2 *\left(\mathrm{E}_{\mathrm{Hf}}+\mathrm{E}_{\mathrm{Nb}}+\mathrm{E}_{\mathrm{Ti}}+\mathrm{E}_{\mathrm{V}}+\mathrm{E}_{\mathrm{Zr}}\right)
$$

To calculate the formation enthalpy with respect to pure elements, we used the VASP energies for pure elements from Ref.[materialsproject.org]

$\mathrm{E}_{\mathrm{Hf}}=-9.9543 \mathrm{eV} /$ atom,

$\mathrm{E}_{\mathrm{Nb}}=-10.1131 \mathrm{eV} /$ atom,

$\mathrm{E}_{\mathrm{Ti}}=-7.8971 \mathrm{eV} /$ atom,

$\mathrm{E}_{\mathrm{V}}=-9.0866 \mathrm{eV} /$ atom,

$\mathrm{E}_{\mathrm{Zr}}=-8.5475 \mathrm{eV} /$ atom 
Using these values, we got:

$\mathrm{H}_{\text {rnd_unrelax }}=0.26 \mathrm{meV} /$ atom - formation enthalpy for random structure without relaxation

$\mathrm{H}_{\text {rnd_relax }}=0.138 \mathrm{meV} /$ atom - formation enthalpy for random structure after relaxation

$\mathrm{H}_{\text {sep_unrelax }}=0.305 \mathrm{eV} /$ atom - formation enthalpy for separated structure without relaxation

$\mathrm{H}_{\text {sep_relax }}=0.117 \mathrm{eV} /$ atom - formation enthalpy for separated structure without relaxation

That is all the HEA structures are thermodynamically unstable with respect to decomposition into pure elements. However, the instability is decreased by at least $50 \%$ due to atomic relaxations. We also notice that pressure turned out to be nearly insensitive to the distribution of atoms.

Interesting that for unrelaxed bcc structures the random alloy is lowest in energy. But with relaxations allowed there is a tendency to decompose the structure into separated elements. However, the calculations have been carried out at $\mathrm{o} \mathrm{K}$, the energy difference is not big, so it is possible that at a higher temperature the free energy values of the two structures will be close.

Next, we considered how the atomic relaxation affects the volumes of intrinsic voids where interstitial impurities can go. As is well known, there are tetrahedral and octahedral voids in the bcc structure. They are 12 and 6 per cubic cell with 2 atoms/cell. That is there is some limited amount of void types defined by their position in the cell, and all the voids of the same type are equivalent in the unrelaxed bcc structure. When different elements are present on different bcc atomic positions, and relaxation of atomic positions happens in a different way depending on the local environment of particular atoms; distribution of sizes and shapes of interstitial sites (voids) is to be observed.

A particular type of voids (tetra or octa) can have different preferability for the occupation by interstitial impurities, depending on their size. We do not address this question, but rather calculate distribution of volumes for both cases. We started with the tetrahedral voids.

\section{Tetrahedral voids}

All tetrahedral voids: are of type [ $\left.\begin{array}{lll}0 & 1 / 2 & 1 / 4\end{array}\right]$, i.e. 4 such voids in each of the faces. The general view is given in Fig. 8.3. 

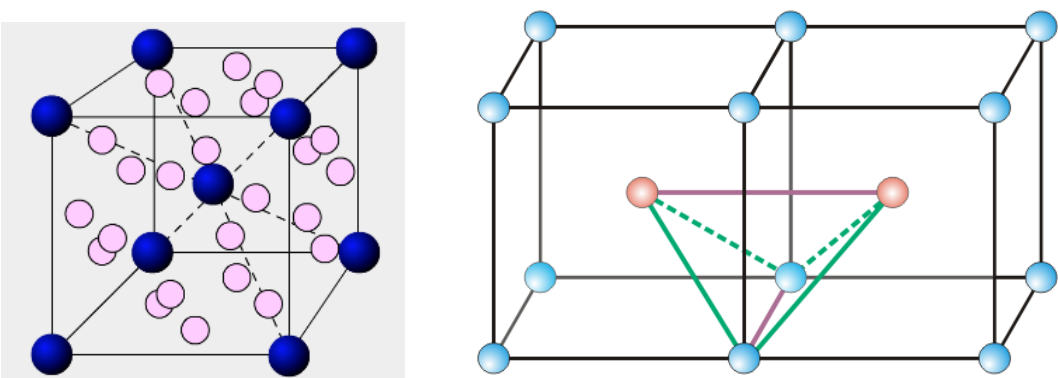

Fig. 8.3. Tetra voids in the bcc structure. Positions of the centres (left panel) and atoms defining one tetra void (right panel).

The effective number of voids is obviously 4 (voids per face) 6 (the number of faces) / 2 (each void belongs to 2 unit cells) $=24 / 2=12$.

All the positions for these voids can be straightforwardly identified in a unit cell or a supercell. Important notice is that in bcc all the voids are irregular, i.e. the distances between the atoms surrounding the void are not equal as in regular tetrahedra or octahedra. Accordingly, to calculate their volumes from the sizes of the edges of a tetrahedron or octahedron is not that straightforward as for regular voids. However, for a general tetrahedron the volume can be calculated numerically using a technique similar to the tetrahedron method used by standard DFT codes for the Brillouin zone integration.

We calculated the volumes for both SQSs, the random and the clustered ones. The results are rather interesting. First of all, due to relaxation we get a Gaussian-like distribution of the void volumes (see Fig. 8.4and 8.5).

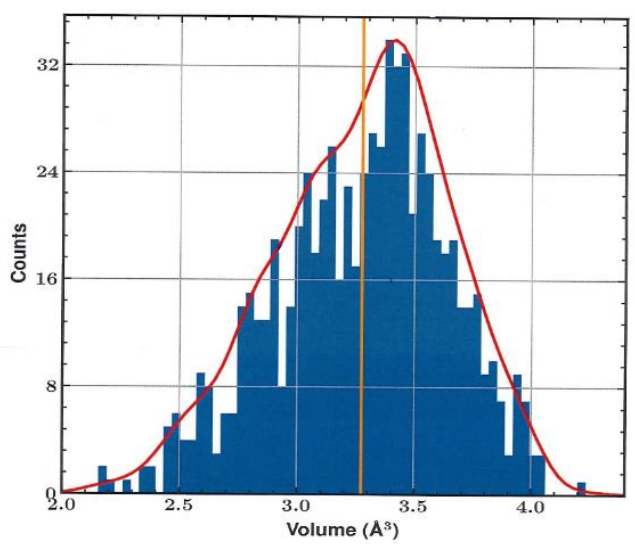

Fig. 8.4. Histogram of the distribution of volumes of tetra voids in the case of random alloy 
The random atomic distribution (Fig. 8.4) results in the volume distribution close to the Gaussian but visibly assymmetric. The vertical line in Fig. 8.4 is the volume of this type of the void that we get in the unrelaxed structure of the same cell volume. There all the volumes, are, of course, equal. In the relaxed structure the maximum is shifted to the right, i.e. to larger volumes. It is important that there are volumes of up to $\Delta \mathrm{V}=22 \%$ larger than in a unrelaxed structure.

The case for the clustered structure is similar $(\Delta \mathrm{V} \sim 25 \%)$. But there is an important difference. The picture is better described with 5 gaussians (we should recall that we have 5 element clustering), see Fig. 8.5.

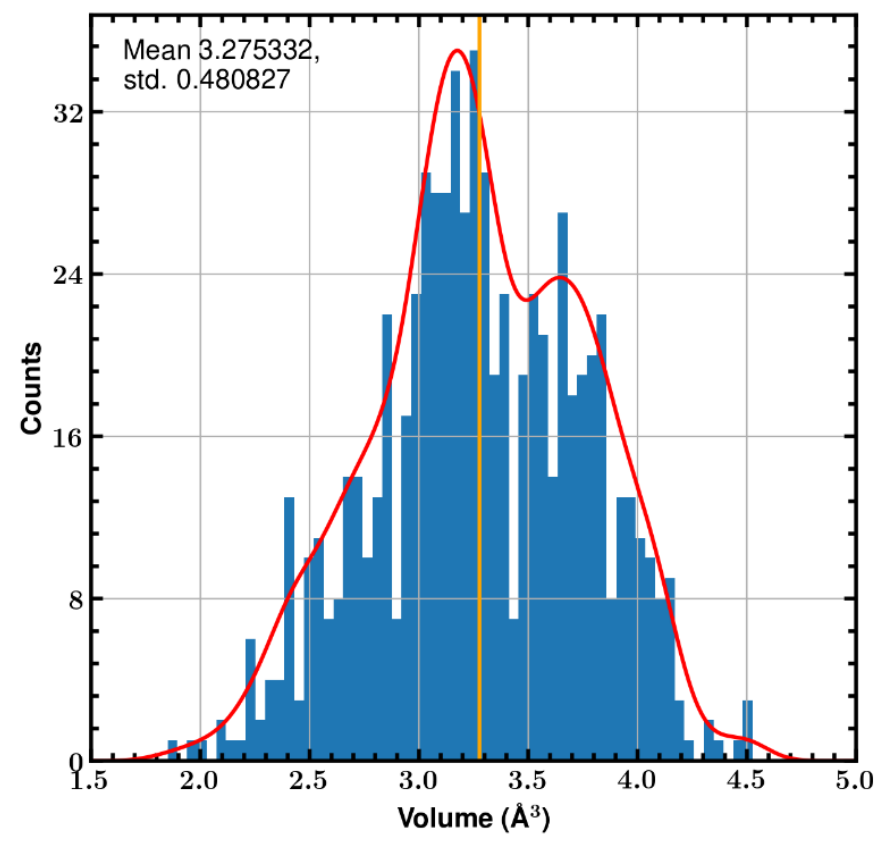

Fig. 8.5. Histogram of the distribution of volumes of tetra voids in the case of clustered structure

We note that the largest maximum is shifted to smaller volumes, and the second one to larger volumes.

\section{Octahedral voids}

For a general octahedron the situation is more difficult and very nontrivial but the problem is also solvable numerically. 
There are 2 types of octahedral voidsin bcc. The first one is at the positions of type $\left[\begin{array}{lll}1 / 2 & 0 & 1 / 2\end{array}\right]$ and the second one is at the positions of type $\left[\begin{array}{lll}0 & 0 & 1 / 2\end{array}\right]$ (see Fig. 8.6), i.e. 6 and 12, respectively(18 in total).

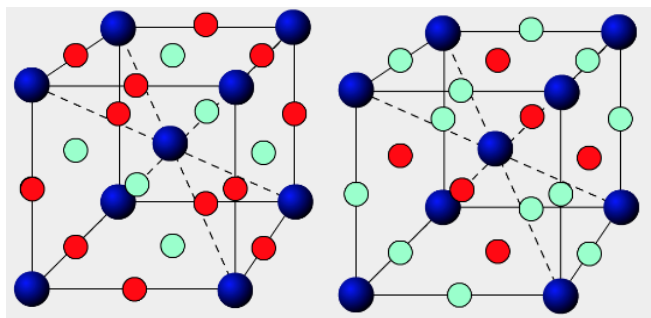

Fig. 8.6. Illustration of octhedral voids in the bec structure.

There are 12 edges. So the effective number of voids (at the edges, i.e. of type [0 $01 / 2$ ]) per cube is $12 / 4=3$. The number of octahedral voids per cell is 3 (at faces, of type [ $1 / 201 / 2$ ]) +3 (at edges) $=6$ per cell. Since a bcc unit cell has 2 atoms per cell, the number of octahedral voids per atom is $6 / 2=3$ per atom.

The general view of an octahedral void is given in Fig. 8.7

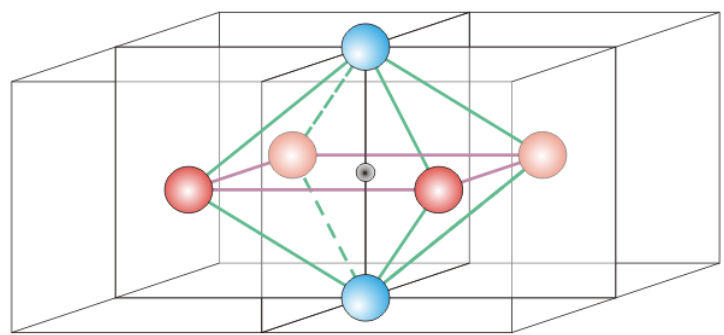

Fig. 8.7. Illustration of octhedral voids

The results for the [0 o $1 / 2$ ] void are shown in Figs. 8.8 and 8.9. Though the mean value is basically unaffected by the atomic relaxation, the distribution over volumes is large. It is also noticeable that in the case of random distribution of elements the void volumes are described by a 2-Gaussian pictures, whereas in the clustered case at least 5 Gaussians are needed. 


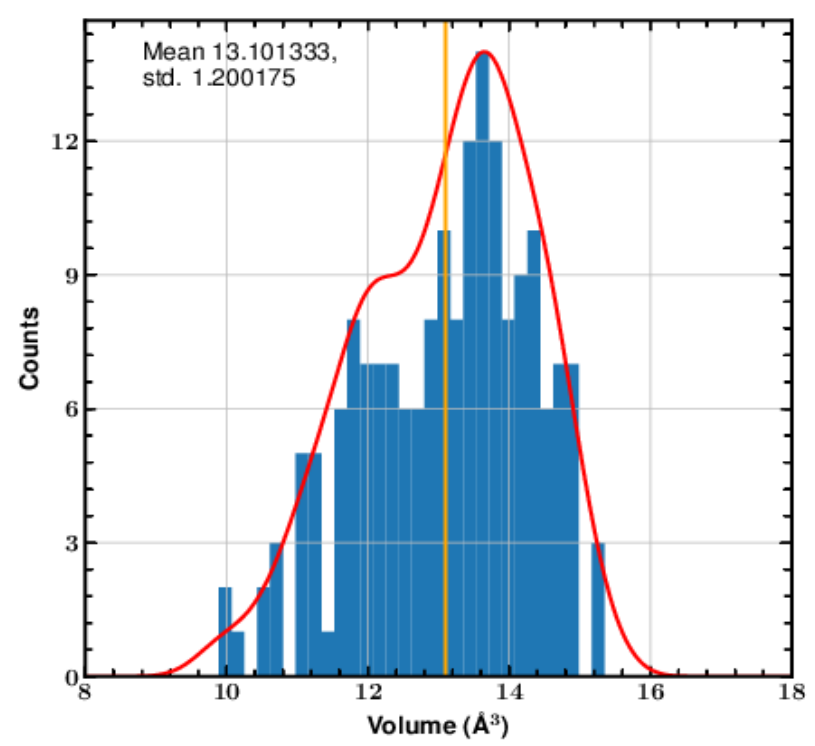

Fig. 8.8. Histogram of the distribution of volumes of octahedral voids of type $[001 / 2]$ in the case of random structure

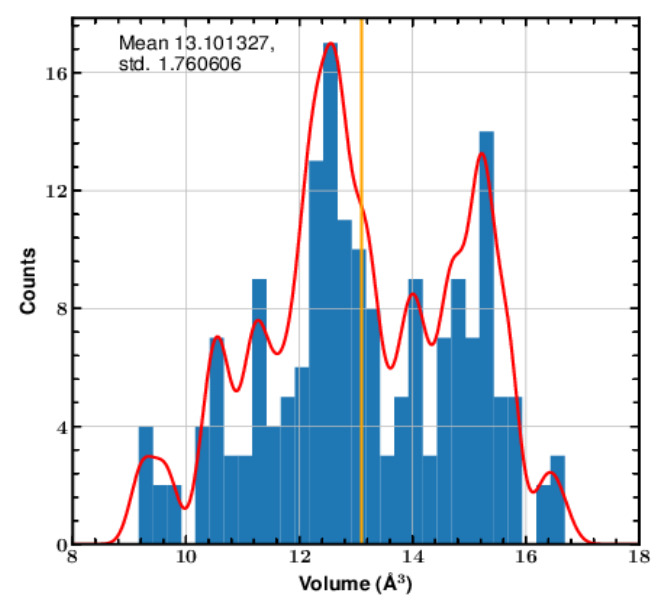

Fig. 8.9. Histogram of the distribution of volumes of octahedral voids of type [o $01 / 2$ ] in the case of clustered structure

The case of the octa void type [ $1 / 201 / 2$ ] is presented in Figs. 8.10 and 8.11. Different for the other octa void type, relaxation here very slightly (by $0.1 \%$ ) shifts the mean volume to larger numbers in the case of the 
random atomic distribution and to the smaller numbers in the clustered case.

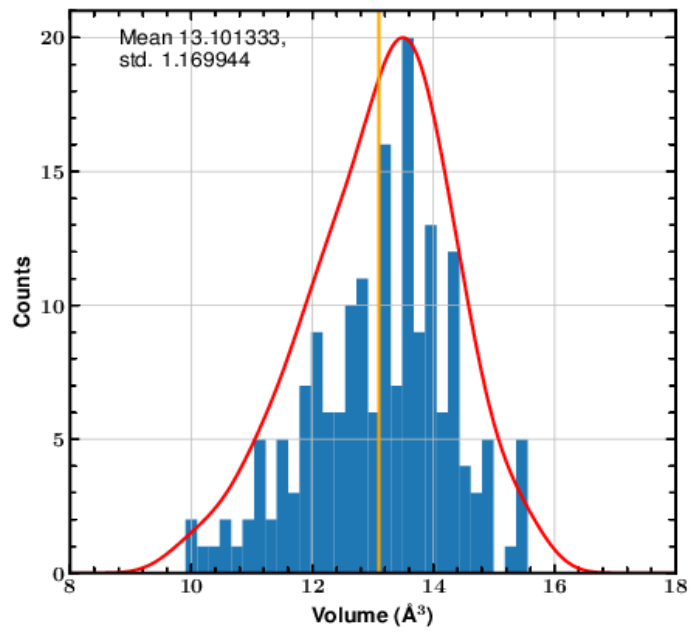

Fig. 8.10. Histogram of the distribution of volumes of octahedral voids of type $\left[\begin{array}{lll}1 / 2 & 0 & 1 / 2\end{array}\right]$ in the case of random structure

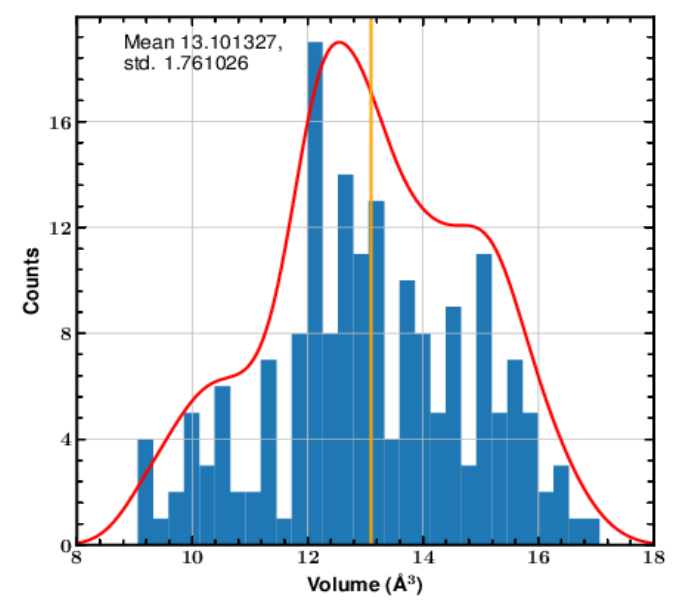

Fig. 8.11. Histogram of the distribution of volumes of octahedral voids of type $[1 / 201 / 2]$ in the case of clustered structure

This type of voids shows greater variability. For a clustered structure, more components can be observed in the probability density. However, the most important fact is that the volumes of octahedral voids are substantially larger than those of tetrahedral voids and seem to be more affected by the atomic relaxations. 
To conclude, we have considered 2 different characteristic distributions of elements in the equiatomic bcc alloy Hf-Nb-Ti-V-Zr. We have found that both structures should be unstable with respect to decomposition into constituting elements at o K. However, atomic relaxations strongly reduce this tendency. Further, we have considered all types of bcc voids and how their volumes are changed by the atomic relaxations. We observe two main effects. First, the relaxation is important for HEA. In particular, it affects stable atomic distribution. Without atomic relaxations the random alloy like distribution is more energetically stable than the structure with clustering of all the atomic species. However, after atomic relaxations the clustered structure is the lowest in energy. Second, the distribution of volumes of voids is strongly affected by the relaxation. There are voids that have up to $25 \%$ volume increase in cases of both tetra and octa voids. However, volume distribution is well described by single and double Gaussians in the case of tetra random alloy and clustered structures, respectively; while double and five-folded Gaussians are needed to describe the distribution of octa voids in the case of tetra random alloy and clustered structures, respectively. Therefore, the atomic relaxations are expected to play different role for incorporating smaller or larger interstitial impurities, like, for example, hydrogen and carbon. 


\section{Chapter 8}

\section{Summary}

In this thesis I studied properties of Ti and its alloys related to a number of applications.

In particular, bcc Ti-V alloys are of practical interest for aerospace and automotive industries, medicine, as well as for various high temperature applications. However, their thermodynamic properties were unexplored from first principles due to dynamical instability at low temperatures. I investigated elastic properties of the bcc Ti-V alloys from first principles in the entire range of concentrations using the PAW and the EMTO methods. The substitutional disorder in the two methods was modeled using special quasi-random structure technique and the coherent potential approximation, respectively. I demonstrated that the PAW-SQS method reproduces the mechanical instability of Ti-rich alloys and therefore allows not only to obtain predictions of the values of elastic moduli in mechanically stable phases, but also to estimate the boundaries of the stability region. In the region of mechanical stability of bec Ti-V alloys, the EMTO-CPA method predicts the elastic moduli in agreement with more accurate theoretical methods. It can be used for an efficient study of concentration dependencies of elastic constants in multicomponent crystalline systems, since this method requires substantially less computational resources. The values of the elastic constants can be refined for alloys of particular interest with, e.g. PAW-SQS calculations, minimizing the total resources needed for the modeling. The described computational strategy simplifies the process of high-throughput calculating the elastic constants for disordered alloys needed, e.g. for the next generation of data-bases of materials parameters.

The results obtained in this study indicate an increase of the mechanical stability of Ti-V alloys with increasing vanadium content. It is shown that the $\beta$ phase of Ti stabilizes when vanadium content approaches 20 at. $\%$. My calculations suggest new opportunities for solving technologically relevant problems. For example, we have demonstrated that in the region of mechanical instability a significant reduction of the elastic moduli $E$ and $G$ are observed. Thus, it is possible to design materials with low values of, for instance, the Young's modulus, by optimizing the alloy composition in such a way as to be as close as possible to the mechanical instability, still remaining within the region of mechanically stable alloy 
compositions. Alloys with low values of the Young's modulus ( $50 \mathrm{GPa}$ ) are of high interest for, e.g. biomedical applications. Though $\mathrm{V}$ is not biocompatible, the strategy proposed above appears to be quite general, and can be used for a design of Ti-based alloys with biocompatible elements.

Further the elastic properties of the bcc Ti-V alloys were investigated as a function of $\mathrm{V}$ concentration from first principles, as well as experimentally, focusing on the alloy composition in a vicinity of the mechanical instability of the bcc phase. Single crystal elastic constants were calculated, and the polycrystalline averages for Young's modulus, $\mathrm{E}$, and for shear modulus, $G$, were obtained. A series of Ti-V alloys with $0-30$ at. \% of $\mathrm{V}$ was synthesized by arc-melting $\mathrm{Ti}$ and $\mathrm{V}$ pieces in an $\mathrm{Ar}$ atmosphere. The composition of the melted samples was determined with $\mathrm{X}$-ray fluorescence, while the crystal structure of the samples was determined with X-ray diffraction. Elastic moduli were determined by nanoindentation. Good qualitative agreement between theory and experiment allowed us to investigate the behavior of the elastic moduli of the bcc phase at $\mathrm{V}$ concentrations as low as 22 at $\% \mathrm{~V}$. Theoretically we demonstrate that mechanical stabilization of bcc Ti-V alloys occurs at 18 at. \% of V. Low values of the Young's modulus in the vicinity of the mechanical instability are confirmed both in theory and in experiment. In addition, theoretical simulations predict very high anisotropy of the Young's modulus in these alloys close to the instability. We conclude that tuning the alloy composition to values as close as possible to mechanical instability could be used as a principle to design materials with specific values of the elastic moduli suitable for the broad range of applications.

Next, the enthalpies of bcc Ti-V alloys were calculated from first principles at 0 and $1300 \mathrm{~K}$ as a function of concentration using static and molecular dynamics simulations. We found that enthalpies of bcc Ti-V alloys are positive in the whole concentration range of vanadium at $0 \mathrm{~K}$, though calculations with static relaxation of atomic positions predict negative values at low vanadium concentration. However, if one considers the formation enthalpy at $0 \mathrm{~K}$ with respect to the stable hcp Ti, it becomes positive at all $\mathrm{V}$ concentrations. Calculations of the formation enthalpy at $1300 \mathrm{~K}$ give much better agreement with the experimental data and show that it is important to take into account thermal vibrations of the atoms. 
Considering the effect of alloying with pure $\mathrm{Ti}$, NiAl was taken as an important system for applications. Our calculations show that such alloying leads to a decrease of the material elastic modulus. For the uncertainty of the example, for $\mathrm{Ni}\left(\mathrm{Al}_{50} \mathrm{Ti}_{50}\right) \mathrm{E}$ is predicted to decrease to $182 \mathrm{GPa}$ in EMTO calculations and to $150 \mathrm{GPa}$ ( PAW calculations. Most importantly, the variation of the Young's modulus in $\mathrm{Ni}\left(\mathrm{AI}_{50} \mathrm{TI}_{50}\right)$ alloy in comparison to NiAl compound is in very good agreement between theoretical methods. One can quantitatively analyze the effect of alloying on other elastic moduli, such as elastic constants $\mathrm{C}_{11}, \mathrm{C}_{12}, \mathrm{C}_{44}$, bulk modulus B, Poisson's ratio v, shear modulus G, Pugh's coefficient k, and Cauchy pressure $\mathrm{P}_{\mathrm{c}}$. Moreover, the calculations reveal that $\mathrm{v}$ increases with increase of $\mathrm{Ti}$ concentration. It is worth noting that $\mathrm{k}$ for the $\mathrm{Ni}(\mathrm{Al}$ 0.5 Ti 0.5 ) alloy is 1.5 times smaller and the value of $\mathrm{P}_{c}$ is increased nearly four times compared with these values for $\mathrm{B} 2 \mathrm{NiAl}$. These results suggest the possibility of improving the ductile properties of $\mathrm{NiAl}$ intermetallics by its alloying with Ti. The observed change in the elastic properties may indicate a change in the character of the chemical bonding of NiAl with increasing concentration of the alloying element Ti. It is known that B2 NiAl has the mixed type of interactions: the metallic bonding is predominant though covalent and ionic bonding are present as well. The decreasing value of $\mathrm{C}_{44}$ and improvement of the ductility characteristics with $\mathrm{Ti}$ addition indicates an increase in the degree of the metallic component in interatomic bonding. I note that while EMTO calculations predict better values of the Young modulus for the alloy in comparison to our experiment, PAW appears to be somewhat more reliable in predicting the elastic modulus concentration variation. Moreover, within the accuracy offered by the phenomenological criterion of brittle vs ductile behavior, we can qualitatively analyze the effect of alloying on the ductility characteristics.

Further, bcc-phase of pure $\mathrm{Ti}$, which is an unstable system at low temperatures, was studied. AIMD simulations were applied to calculate the temperature dependent elastic constants of pure bcc Ti. While the system is unstable at $\mathrm{T}=300 \mathrm{~K}$, average stresses demonstrate large oscillations at $\mathrm{T}=600 \mathrm{~K}$, and at $\mathrm{T}=900 \mathrm{~K}$ the system is stabilized. That can be seen after relatively small number of time steps. We reproduced the mechanical stabilization of pure bcc Ti under heating. Bcc structure of $\mathrm{Ti}$ is stable when the temperature is above $700 \mathrm{~K}$ according to the requirements of the mechanical stability for a cubic crystal. The mechanical stability at $700 \mathrm{~K}$ is not sufficient to stabilize the bcc phase with respect to the hcp phase. The thermodynamic stability of the bcc phase is achieved at higher temperature, $\mathrm{T}=1155 \mathrm{~K}$. 
Next, diffusion in bcc Ti was investigated. There is an anomaly of nonArrhenius behavior of diffusion coefficient in bcc Ti when approaching the temperature corresponding to the unstable region. Our studies were aimed at determining whether the non-Arrhenius vacancy migration rates are strong in the vicinity of the dynamic instability. We wanted to see how the transition from the unstable to stable bcc phase could affect its self-diffusion. From temperatures of $\sim 80 \%$ of the Ti melting point, concerted migration in defect-free lattice regions becomes the dominant diffusion mechanism, that is, the calculated self-diffusion coefficient is essentially unaffected by the presence of point defects. We obtained rate of a diffusion process at temperature $1800 \mathrm{~K}$. We obtain rapid spontaneous formation of Frenkel pairs in defect-free bcc Ti. It is indicative of very low defect formation energies in strongly anharmonic systems. With enough excess energy, an atom jumps into the interstitial space and does not return back but rather goes on in the interstitial space. More important, the spontaneous formation of Frenkel pairs (with no quick annihilation (recombination) of vacancy or interstitials) is done in a concerted manner, so collective motion of Ti atoms is observed. This is the reaction pathway for formation of defects in a defect free lattice. The pathway itself is of interest: thermodynamics tells that any lattice contains defects but does not tell how these defects are actually formed. Therefore we revealed an interesting mechanism for high-temperature diffusion in bcc titanium.

Further, we studied from first principles the effect of atomic relaxations on formation enthalpies and distributions of volumes of tetra and octa voids in body-centered cubic equiatomic alloy Ti-Hf-Nb-V-Zr. We found that random or clustered distribution of elements in this alloy should be unstable with respect to decomposition into constituting elements at o K. However, atomic relaxations strongly reduce this tendency. We found two main effects. First, the relaxation is important for HEA. In particular, it affects stable atomic distribution. Without atomic relaxations the random alloy like distribution is more energetically stable than the structure with clustering of all the atomic species. However, after atomic relaxations the clustered structure is the lowest in energy. Second, the distribution of volumes of voids is strongly affected by the relaxation. There are voids that have up to $25 \%$ volume increase in cases of both tetra and octa voids. However, volume distribution is well described by single and double Gaussians in the case 
of tetra random alloy and clustered structures, respectively; while double and five-folded Gaussians are needed to describe the distribution of octa voids in the case of tetra random alloy and clustered structures, respectively. Therefore, the atomic relaxations are expected to play different role for incorporating smaller or larger interstitial impurities, like, for example, hydrogen and carbon. 



\section{References}

[1] R. Chinnappan, B. K. Panigrahi, and A. van de Walle, "First-principles study of phase equilibrium in Ti-V, Ti-Nb, and Ti-Ta alloys," Calphad, vol. 54, pp. 125-133, Sep. 2016.

[2] M. Sluiter and P. E. A. Turchi, "Phase stability in Ti-V and Ti-Cr alloys: A theoretical investigation," Phys. Rev. B, vol. 43, no. 15, pp. 12251-12266, May 1991.

[3] A. Kostov and D. Živković, "Thermodynamic analysis of alloys Ti-Al, Ti-V, Al-V and TiAl-V," J. Alloys Compd., vol. 460, no. 1-2, pp. 164-171, Jul. 2008.

[4] L.-J. Gui et al., "Behaviors of alloying element titanium in vanadium: From energetics to tensile/shear deformation," Comput. Mater. Sci., vol. 77, pp. 348-354, Sep. 2013.

[5] S. Das, "Physical Aspects of Process Control in Selective Laser Sintering of Metals," Adv. Eng. Mater., vol. 5, no. 10, pp. 701-711, Oct. 2003.

[6] S. Suwarno, J. K. Solberg, J. P. Maehlen, B. Krogh, and V. A. Yartys, "The effects of rapid solidification on microstructure and hydrogen sorption properties of binary BCC Ti-V alloys," J. Alloys Compd., vol. 582, pp. 540-546, Jan. 2014.

[7] T. Ozaki, H. Matsumoto, S. Watanabe, and S. Hanada, "Beta Ti Alloys with Low Young's Modulus," Mater. Trans., vol. 45, no. 8, pp. 2776-2779, 2004.

[8] N. V. Skripnyak, A. V. Ponomareva, M. P. Belov, and I. A. Abrikosov, "Ab initio calculations of elastic properties of alloys with mechanical instability: Application to BCC Ti-V alloys," Mater. Des., vol. 140, pp. 357-365, Feb. 2018.

[9] X. Li, C. Zhang, J. Zhao, and B. Johansson, "Mechanical properties and defective effects of bcc $\mathrm{V}-4 \mathrm{Cr}-4 \mathrm{Ti}$ and $\mathrm{V}-5 \mathrm{Cr}-5 \mathrm{Ti}$ alloys by first-principles simulations," Comput. Mater. Sci., vol. 50, no. 9, pp. 2727-2731, Jul. 2011.

[10] M. Born and R. Oppenheimer, "Zur Quantentheorie der Molekeln,” Ann. Phys., vol. 389, no. 20, pp. 457-484, 1927.

[11] P. Hohenberg and W. Kohn, "Inhomogeneous Electron Gas," Phys. Rev., vol. 136, no. 3B, pp. B864-B871, Nov. 1964.

[12] W. Kohn and L. J. Sham, "Self-Consistent Equations Including Exchange and Correlation Effects," Phys. Rev., vol. 140, no. 4A, pp. A1133-A1138, Nov. 1965.

[13] K. Burke and L. O. Wagner, "DFT in a nutshell," Int. J. Quantum Chem., vol. 113, no. 2, pp. 96-101, Jan. 2013.

[14] A. D. Becke, "Perspective: Fifty years of density-functional theory in chemical physics," $J$. Chem. Phys., vol. 140, no. 18, p. 18A301, May 2014.

[15] J. P. Perdew et al., "Atoms, molecules, solids, and surfaces: Applications of the generalized gradient approximation for exchange and correlation," Phys. Rev. B, vol. 46, no. 11, pp. 6671-6687, Sep. 1992.

[16] A. D. Becke, "Density-functional exchange-energy approximation with correct asymptotic behavior," Phys. Rev. A, vol. 38, no. 6, pp. 3098-3100, Sep. 1988.

[17] D. C. Langreth and M. J. Mehl, "Beyond the local-density approximation in calculations of ground-state electronic properties," Phys. Rev. B, vol. 28, no. 4, pp. 1809-1834, Aug. 1983. 
[18] J. P. Perdew, K. Burke, and M. Ernzerhof, "Generalized Gradient Approximation Made Simple [Phys. Rev. Lett. 77, 3865 (1996)],” Phys. Rev. Lett., vol. 78, no. 7, pp. 1396-1396, Feb. 1997.

[19] L. Vitos, Computational Quantum Mechanics for Materials Engineers. London: Springer London, 2007.

[20] L. Vitos, I. A. Abrikosov, and B. Johansson, "Coherent Potential Approximation Within the Exact Muffin-Tin Orbitals Theory,” in Complex Inorganic Solids, Boston, MA: Springer US, pp. 339-352.

[21] P. E. Blöchl, "Projector augmented-wave method," Phys. Rev. B, vol. 50, no. 24, pp. 1795317979, Dec. 1994.

[22] G. Kresse and D. Joubert, "From ultrasoft pseudopotentials to the projector augmentedwave method," Phys. Rev. B, vol. 59, no. 3, pp. 1758-1775, Jan. 1999.

[23] D. Frenkel and B. Smit, Understanding Molecular Simulation, Second Edition: From Algorithms to Applications (Computational Science). 2001.

[24] S. Nosé, "A unified formulation of the constant temperature molecular dynamics methods," J. Chem. Phys., vol. 81, no. 1, pp. 511-519, Jul. 1984.

[25] W. G. Hoover, "Canonical dynamics: Equilibrium phase-space distributions," Phys. Rev. $A$, vol. 31, no. 3, pp. 1695-1697, Mar. 1985.

[26] G. J. Martyna, M. L. Klein, and M. Tuckerman, "Nosé-Hoover chains: The canonical ensemble via continuous dynamics,” J. Chem. Phys., vol. 97, no. 4, pp. 2635-2643, Aug. 1992.

[27] L. Verlet, "Computer 'Experiments' on Classical Fluids. I. Thermodynamical Properties of Lennard-Jones Molecules,” Phys. Rev., vol. 159, no. 1, pp. 98-103, Jul. 1967.

[28] A. Zunger, S.-H. Wei, L. G. Ferreira, and J. E. Bernard, "Special quasirandom structures," Phys. Rev. Lett., vol. 65, no. 3, pp. 353-356, Jul. 1990.

[29] D. B. Laks, L. G. Ferreira, S. Froyen, and A. Zunger, "Efficient cluster expansion for substitutional systems," Phys. Rev. B, vol. 46, no. 19, pp. 12587-12605, Nov. 1992.

[30] F. Tasnádi, M. Odén, and I. A. Abrikosov, "Ab initio elastic tensor of cubic Ti o.5 Al $0.5 \mathrm{~N}$ alloys: Dependence of elastic constants on size and shape of the supercell model and their convergence," Phys. Rev. B, vol. 85, no. 14, p. 144112, Apr. 2012.

[31] M. Moakher and A. N. Norris, "The Closest Elastic Tensor of Arbitrary Symmetry to an Elasticity Tensor of Lower Symmetry,” J. Elast., vol. 85, no. 3, pp. 215-263, Oct. 2006.

[32] D. G. Pettifor, "Theoretical predictions of structure and related properties of intermetallics," Mater. Sci. Technol., vol. 8, no. 4, pp. 345-349, Apr. 1992.

[33] S. F. Pugh, "XCII. Relations between the elastic moduli and the plastic properties of polycrystalline pure metals," London, Edinburgh, Dublin Philos. Mag. J. Sci., vol. 45, no. 367, pp. 823-843, Aug. 1954.

[34] G. V Sin ko and N. A. Smirnov, “On elasticity under pressure,” J. Phys. Condens. Matter, vol. 16, no. 45, pp. 8101-8104, Nov. 2004. 
[35] G. V. Sin'ko, "Ab initio calculations of the second-order elastic constants of crystals under arbitrary isotropic pressure,” Phys. Rev. B, vol. 77, no. 10, p. 104118, Mar. 2008.

[36] D. Psiachos, T. Hammerschmidt, and R. Drautz, "Ab initio study of the modification of elastic properties of $\alpha$-iron by hydrostatic strain and by hydrogen interstitials," Acta Mater., vol. 59, no. 11, pp. 4255-4263, Jun. 2011.

[37] R. Hill, “The Elastic Behaviour of a Crystalline Aggregate,” Proc. Phys. Soc. Sect. A, vol. 65, no. 5, pp. 349-354, May 1952.

[38] N. Frantsevich, F. F. Voronov, and S. A. Bakuta, Handbook on Elastic Constants and Moduli of Elasticity for Metals and Nonmetals. Kiev: Naukova Dumka, 1982.

[39] D. Raabe, B. Sander, M. Friák, D. Ma, and J. Neugebauer, “Theory-guided bottom-up design of $\beta$-titanium alloys as biomaterials based on first principles calculations: Theory and experiments," Acta Mater., vol. 55, no. 13, pp. 4475-4487, Aug. 2007.

[40] M. Friák et al., "Theory-Guided Materials Design of Multi-Phase Ti-Nb Alloys with BoneMatching Elastic Properties,” Materials (Basel)., vol. 5, no. 10, pp. 1853-1872, Oct. 2012.

[41] T. D. Shermergor, Teoriya uprugosti mikroneodnorodnykh sred. Nauka, 1977.

[42] J. Korll' ar, L. Vitos, and H. L. Skriver, "The Uses of the LMTO Method, Lecture Notes in Physics," in Electronic Structure and Physical Properties of Solids, H. Dreyss'e, Ed. Berlin: Springer, 2000, p. 85.

[43] G. Kresse and J. Furthmüller, "Efficiency of ab-initio total energy calculations for metals and semiconductors using a plane-wave basis set," Comput. Mater. Sci., vol. 6, no. 1, pp. 15-50, Jul. 1996.

[44] G. Kresse and J. Furthmüller, "Efficient iterative schemes for ab initio total-energy calculations using a plane-wave basis set," Phys. Rev. B, vol. 54, no. 16, pp. 11169-11186, Oct. 1996.

[45] H. Ikehata, N. Nagasako, T. Furuta, A. Fukumoto, K. Miwa, and T. Saito, "First-principles calculations for development of low elastic modulus Ti alloys," Phys. Rev. B, vol. 70, no. 17, p. 174113 , Nov. 2004.

[46] K. W. Katahara, M. H. Manghnani, and E. S. Fisher, "Pressure derivatives of the elastic moduli of BCC Ti-V-Cr, Nb-Mo and Ta-W alloys," J. Phys. F Met. Phys., vol. 9, no. 5, pp. 773-790, May 1979.

[47] D. I. Bolef, R. E. Smith, and J. G. Miller, "Elastic Properties of Vanadium. I. Temperature Dependence of the Elastic Constants and the Thermal Expansion," Phys. Rev. B, vol. 3, no. 12, pp. 4100-4108, Jun. 1971.

[48] X. Li et al., "Elastic properties of vanadium-based alloys from first-principles theory," Phys. Rev. B, vol. 86, no. 1, p. 014105, Jul. 2012.

[49] M. J. Mehl and D. A. Papaconstantopoulos, "Applications of a tight-binding total-energy method for transition and noble metals: Elastic constants, vacancies, and surfaces of monatomic metals," Phys. Rev. B, vol. 54, no. 7, pp. 4519-4530, Aug. 1996.

[50] M. Jahnátek, M. Krajčí, and J. Hafner, "Interatomic bonding, elastic properties, and ideal strength of transition metal aluminides: A case study for $<$ math display="inline" $><$ mrow $>$ $<$ msub $><$ mi mathvariant="normal" $>\mathrm{Al}</$ mi $><$ mn $>3</$ mn $></$ msub $><$ mrow $>$ $<$ mo $>(</$ mo $><$ mi mathvariant="normal" $>\mathrm{V}</$ mi $><$ mo $>,<$," Phys. Rev. B, vol. 71, no. 2, 
p. 024101, Jan. 2005 .

[51] L. Koči, Y. Ma, A. R. Oganov, P. Souvatzis, and R. Ahuja, "Elasticity of the superconducting metals V, Nb, Ta, Mo, and W at high pressure," Phys. Rev. B, vol. 77, no. 21, p. 214101, Jun. 2008.

[52] Z. T. Y. Liu, B. P. Burton, S. V Khare, and D. Gall, "First-principles phase diagram calculations for the rocksalt-structure quasibinary systems TiN-ZrN, TiN-HfN and $\mathrm{ZrN}-$ HfN,” J. Phys. Condens. Matter, vol. 29, no. 3, p. 035401, Jan. 2017.

[53] P. Söderlind, A. Landa, L. H. Yang, and A. M. Teweldeberhan, "First-principles phase stability in the Ti-V alloy system," J. Alloys Compd., vol. 581, pp. 856-859, Dec. 2013.

[54] A. V. Dobromyslov, G. V. Dolgikh, Y. Dutkevich, and T. L. Trenogina, "Phase and structural transformations in Ti-Ta alloys," Phys. Met. Metallogr., vol. 107, no. 5, pp. 502-510, May 2009.

[55] J. T. Lenkkeri and E. E. Lahteenkorva, "An investigation of elastic moduli of vanadiumchromium alloys,” J. Phys. F Met. Phys., vol. 8, no. 8, pp. 1643-1651, Aug. 1978.

[56] L. Vočadlo, "Ab initio calculations of the elasticity of iron and iron alloys at inner core conditions: Evidence for a partially molten inner core?,” Earth Planet. Sci. Lett., vol. 254, no. 1-2, pp. 227-232, Feb. 2007.

[57] H. Ogi, S. Kai, H. Ledbetter, R. Tarumi, M. Hirao, and K. Takashima, "Titanium's hightemperature elastic constants through the hcp-bcc phase transformation," Acta Mater., vol. 52, no. 7, pp. 2075-2080, Apr. 2004.

[58] Y. F. Ye, Q. Wang, J. Lu, C. T. Liu, and Y. Yang, "High-entropy alloy: challenges and prospects," Mater. Today, vol. 19, no. 6, pp. 349-362, 2016.

[59] H. S. Oh et al., "Lattice distortions in the FeCoNiCrMn high entropy alloy studied by theory and experiment," Entropy, vol. 18, no. 9, pp. 1-9, 2016. 


\section{List of included publications}

[I] Ab initio calculations of elastic properties of alloys with mechanical instability: Application to BCC Ti-V alloys

N. V. Skripnyak, A. V. Ponomareva, M. P. Belov, and I. A. Abrikosov

Mater. Des., vol. 140, pp. 357-365, 2018.

Majority of the calculations, analyses of results, literature survey; main part of the manuscript.

[II] Superioniclike diffusion in an elemental crystal: Bcc Titanium

D. G. Sangiovanni, J. Klarbring, D. Smirnova, N. V. Skripnyak, D. Gambino, M. Mrovec, S. I. Simak, and I. A. Abrikosov

Phys. Rev. Lett., vol. 123, no. 10, 2019.

Part of the calculations, the manuscript was written jointly.

[III] Mixing enthalpies of alloys with dynamical instability: bcc Ti-V system N. V. Skripnyak, A. V. Ponomareva, M. P. Belov, E.A. Syutkin, A.V. Khvan, A.T. Dinsdale, I. A. Abrikosov

Acta Mater., published online, https://doi.org/10.1016/j.actamat.2020.01.056, 2020.

Part of the theoretical calculations, analyses of results; the manuscript was written jointly.

[IV] Achieving low elastic moduli of bcc Ti-V alloys in vicinity of mechanical instability

N. V. Skripnyak, S. I. Simak, A. V. Ponomareva, F. Tasnadi, J. Löfstrand, P. Berastegui, U. Jansson, I. A. Abrikosov.

manuscript, 2020.

Leading theoretical author, part in problem formulation, majority of the calculations, analyses of results, literature survey; main part of the manuscript.

[V] Effect of atomic relaxations on the atomic distributions in $\mathrm{Hf}-\mathrm{Nb}-\mathrm{Ti}-\mathrm{V}-\mathrm{Zr}$ high entropy alloys

N. V. Skripnyak, S. Filippov, I. A. Abrikosov

manuscript, 2020.

All first-principles calculations, analyses of results, literature survey; the manuscript was written by me. 



\section{List of publications}

[1] V. A. Skripnyak, V. V Skripnyak, E. G. Skripnyak, and N. V Skripnyak, "Modelling of the mechanical response of $\mathrm{Zr}-\mathrm{Nb}$ and Ti-Nb alloys in a wide temperature range," Int. J. Mech. Mater. Des., 2019.

[2] V. A. Skripnyak, N. V Skripnyak, E. G. Skripnyak, and V. V Skripnyak, "Influence of grain size distribution on the mechanical behavior of light alloys in wide range of strain rates," AIP Conf. Proc., vol. 1793, 2017.

[3] N. V Skripnyak, E. S. Emelyanova, V. A. Skripnyak, and E. G. Skripnyak, "Damage of highchromium steels under deformation in a wide temperature range," AIP Conf. Proc., vol. 1909, 2017.

[4] V. A. Serbenta, N. V Skripnyak, V. A. Skripnyak, and E. G. Skripnyak, "Numerical simulation of the mechanical behavior of ultrafine- and coarse-grained $\mathrm{Zr}$-Nb alloys over a wide range of strain rates," AIP Conf. Proc., vol. 1909, 2017.

[5] V. A. Skripnyak, E. S. Emelyanova, M. V Sergeev, N. V Skripnyak, and O. S. Zinovieva, "Strength and plasticity of Fe-Cr alloys," AIP Conf. Proc., vol. 1783, 2016.

[6] K. P. Sidnov et al., "Effect of alloying on elastic properties of ternary Ni-Al-Ti system: Experimental validation,” J. Alloys Compd., vol. 688, pp. 534-541, Dec. 2016.

[7] E. G. Skripnyak, V. V Skripnyak, V. A. Skripnyak, N. V Skripnyak, and I. K. Vaganova, "Brittle or quasi-brittle fracture of ceramic nanocomposites under dynamic loading," ECCOMAS Congr. 2016 - Proc. 7th Eur. Congr. Comput. Methods Appl. Sci. Eng., vol. 1, pp. 366-372, 2016.

[8] N. V Skripnyak, V. V Skripnyak, and V. A. Skripnyak, "Fracture of thin metal sheets with distribution of grain sizes in the layers," ECCOMAS Congr. 2016 - Proc. 7th Eur. Congr. Comput. Methods Appl. Sci. Eng., vol. 1, pp. 355-365, 2016.

[9] A. Y. Nikonov et al., "Calculation of mechanical properties of BCC Ti-Nb alloys," in AIP Conference Proceedings, 2015, vol. 1683, p. 020165.

[10] N. V Skripnyak, "The Features of Fracture Behavior of an Aluminum-Magnesium Alloy AMg6 Under High-Rate Straining,” Russ. Phys. J., vol. 58, no. 5, pp. 691-697, 2015.

[11] N. V Skripnyak, E. G. Skripnyak, V. A. Skripnyak, V. V Skripnyak, and I. K. Vaganova, "Failure mechanisms of light alloys with a bimodal grain size distribution," 11th World Congr. Comput. Mech. WCCM 2014, 5th Eur. Conf. Comput. Mech. ECCM 2014 6th Eur. Conf. Comput. Fluid Dyn. ECFD 2014, pp. 3915-3925, 2014.

[12] N. V Skripnyak, E. G. Skripnyak, V. A. Skripnyak, V. V Skripnyak, and I. K. Vaganova, "Failure mechanisms of light alloys with a bimodal grain size distribution," 11th World Congr. Comput. Mech. 5th Eur. Conf. Comput. Mech. 6th Eur. Conf. Comput. Fluid Dyn. Vols Ii - Iv, pp. 3915-3925, 2014.

[13] V. A. Skripnyak, E. G. Skripnyak, N. V Skripnyak, L. W. Meyer, and N. Herzig, "Fracture of light alloys with structured surface layers under dynamic loadings," 19th Eur. Conf. Fract. Fract. Mech. Durability, Reliab. Safety, ECF 2012, 2012. 



\section{Papers}

The papers associated with this thesis have been removed for copyright reasons. For more details about these see:

http://urn.kb.se/resolve?urn=urn:nbn:se:liu:diva-165270 


\section{FACULTY OF SCIENCE AND ENGINEERING}

Linköping Studies in Science and Technology, Dissertations No. 2064, 2020 Department of Physics, Chemistry, and Biology (IFM)

Linköping University

SE-581 83 Linköping, Sweden

www.liu.se 